\title{
ANALYSIS ON THE CROWN DOMAIN
}

\author{
BernhaRd KRÖTZ AND ERIC OPdAM
}

\begin{abstract}
This paper is a further development of complex methods in harmonic analysis on semi-simple Lie groups $[\mathrm{AG}],[\mathrm{BeR}],[\mathrm{KrS} 1,2]$. We study the growth behaviour of the holomorphic extension of the orbit map of the spherical vector of an irreducible spherical representation of a real reductive group $G$ when approaching the boundary of the crown domain of the Riemannian symmetric space $G / K$. As an application, we prove that Maaß cusp forms have exponential decay.
\end{abstract}

\section{Contents}

3 New Features of $G=\operatorname{Sl}(2, \mathbb{R}) \quad 1338$

3.1 Corner view . . . . . . . . . . . . . . . . . . . . . . . . . . . . . . . . 1338

3.2 Unipotent parameterization . . . . . . . . . . . . . . . . . 1340

4 Properness and Maximality of Holomorphic Extension

Keywords and phrases: Crown domain, harmonic analysis, Maaß cusp forms, hypergeometric functions associated to root systems

AMS Mathematics Subject Classification: 22Exx, 11xx

During the preparation of this paper the second named author was partially supported by a Pionier Grant of the Netherlands Organization for Scientific Research (NWO). Part of this research was carried out in the fall of 2004, during which period both authors enjoyed the hospitality of the Research Institute for the Mathematical Sciences in Kyoto, Japan. It is our pleasure to thank the RIMS for its hospitality and for the stimulating environment it offers. 
6 Sharp Uniform Lower Bound for Holomorphically Extended Orbit Maps of Spherical Representations

7 Sharp Upper Bound for Holomorphically Extended Orbit Maps of Spherical Representations $\quad 1356$

7.0.1 The real rank one case . . . . . . . . . . . . . . . . . . . . 1358

7.0 .2 The complex case . . . . . . . . . . . . . . . . . . . . . 1360

8 Unipotent Model for the Crown Domain $\mathbf{1 3 6 5}$

8.1 The case of real rank one . . . . . . . . . . . . . . . . . . . 1367

8.2 The case where $\Xi$ is a Hermitian symmetric space . . . . . . . . . . . . . 1370

8.2.1 The symplectic group . . . . . . . . . . . . . . . . . . . . 1371

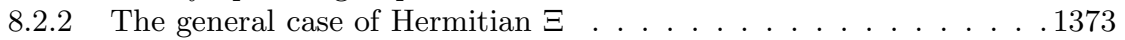

8.3 Some partial results for the special linear groups . . . . . . . . . . . . . 1374

9 Exponential Decay of Maaß Cusp Forms I: The Example of $\begin{array}{lr}G=\operatorname{Sl}(2, \mathbb{R}) & 1377\end{array}$

9.1 Concrete approach . . . . . . . . . . . . . . . . . . . . . 1377

9.2 The method of analytic continuation . . . . . . . . . . . . . . . 1379

10 Exponential Decay of Maaß Cusp Forms II:

The Rank-One Cases

11 Exponential Decay of Maaß Cusp Forms III:

The Higher-Rank Cases 1382

11.1 Refinements of the main estimate . . . . . . . . . . . . . . . . . . . . . . 1386

11.2 A Bergman estimate on the local crown domains . . . . . . . . . . . . . . . . . . . . . . . . . .

11.3 Main estimates in final form . . . . . . . . . . . . . . . . . . . . . . . . . . 1389

12 Final Remarks 1392

12.1 Estimates on Whittaker functionals for $\operatorname{Gl}(n)$ are sharp . . . . . . . . . . 1392

12.2 Automorphic holomorphic triple products . . . . . . . . . . . . . . 1393

12.3 Exponential decay of automorphic triple products. . . . . . . . . . . . . . 1394

13 Appendix: Leading Exponents of Holomorphically Extended Elemen-

$\begin{array}{lr}\text { tary Spherical Functions } & \mathbf{1 3 9 5}\end{array}$

13.1 Exponents and Hyperplane Arrangements . . . . . . . . . . . . . . . . . . 1396

13.2 Harish-Chandra's radial system of differential equations . . . . . . . . . . 1400

13.3 The hypergeometric system of differential equations . . . . . . . . . . . . 1401

13.4 The indicial equation . . . . . . . . . . . . . . . . . . . . . . . . . . 1404

13.5 Hecke algebras and exponents . . . . . . . . . . . . . . . . . . . . . . . . 1408

13.6 Computation of the leading exponents . . . . . . . . . . . . . . . . . 1411

\section{Introduction}

Our concern is with harmonic analysis on a Riemannian symmetric space

$$
X_{\mathbb{R}}=G_{\mathbb{R}} / K_{\mathbb{R}}
$$

of the noncompact type. Here $G_{\mathbb{R}}$ denotes a connected reductive algebraic group and $K_{\mathbb{R}}$ is a maximal compact subgroup thereof. 
Given a $K_{\mathbb{R}}$-spherical irreducible unitary representation $\left(\pi, \mathcal{H}_{\pi}\right)$ of $G_{\mathbb{R}}$ with $K_{\mathbb{R}}$-fixed ray $\mathcal{H}_{\pi}^{K_{\mathbb{R}}}$-fix $=\mathbb{C} v_{K}$, we obtain an $G_{\mathbb{R}}$-equivariant continuous map

$$
i_{\pi}: X_{\mathbb{R}} \rightarrow \mathcal{H}_{\pi}, \quad g K_{\mathbb{R}} \mapsto \pi(g) v_{K} .
$$

We assume that $\pi \neq \mathbf{1}$ is non-trivial and then $i_{\pi}$ is injective. The map $i_{\pi}$ is analytic, hence admits holomorphic extension to a maximal $G_{\mathbb{R}^{-}}$ neighborhood $\Xi_{\pi}$ of $X_{\mathbb{R}}$ in $X_{\mathbb{C}}=G_{\mathbb{C}} / K_{\mathbb{C}}$. It is a remarkable fact that $\Xi_{\pi}$ is independent of the choice of $\pi \neq \mathbf{1}([\mathrm{KrS1} 1,2],[\mathrm{KrOS}])$ and hence defines a natural domain $\Xi$ in $X_{\mathbb{C}}$, referred to as the crown domain. A result in this paper determines the precise growth rate of $\left\|i_{\pi}\right\|$ when approaching the boundary of $\Xi$.

We have to clarify what we understand by the term "approaching the boundary". The crown domain admits a natural Shilov-type boundary [GiK1], referred to as the distinguished boundary $\partial_{d} \Xi$ of $\Xi$. In a first step we give a simple description of $\partial_{d} \Xi$ in terms of the affine Weyl group, hereby extending and unifying results from [GiK1]. At this point it is relevant that the $G_{\mathbb{R}}$-equivalence classes in $\partial_{d} \Xi$ are described by a finite union of Weylgroup orbits.

Given a distinguished boundary point $z \in \partial_{d} \Xi$ and $\left(z_{n}\right) \subset \Xi$ a sequence converging radially to $z$ we are interested in the growth of $\left\|i_{\pi}\left(z_{n}\right)\right\|$ in terms of $\operatorname{dist}\left(z_{n}, z\right)$. We determine

- for fixed $z_{n}$, sufficiently close to $z$, optimal lower exponential bounds for $\left\|i_{\pi}\left(z_{n}\right)\right\|$ in terms of the parameter of $\pi$ and $\operatorname{dist}\left(z_{n}, z\right)$;

- for fixed $\pi$, the precise blow-up rate of $\left\|i_{\pi}\left(z_{n}\right)\right\|$ for $z_{n} \rightarrow z$ in terms of $\operatorname{dist}\left(z_{n}, z\right)$.

We use these results to prove estimates for Maaß automorphic forms. For example, a theorem of Langlands asserts that cuspidal automorphic forms are of rapid decay [L], [H2]. An unpublished theorem of J. Bernstein goes beyond and asserts exponential decay. In this paper we establish precise exponential decay rates. The basic idea of proof goes back to Bernstein and our contribution lies in a incorporation of geometric methods and hard estimates. (J.B. explained to us the case of $G=\mathrm{Sl}(2, \mathbb{R})$, cf. the first half of subsection 9.2.) In particular, we show that the crown domain admits a natural parameterization by unipotent $G_{\mathbb{R}}$-orbits which makes Bernstein's idea work out efficiently.

Finally we wish to point out that we make a detailed study of proper actions of $G_{\mathbb{R}}$ on $X_{\mathbb{C}}$ in section 4 . As a by-product of these investigations we obtain a complex geometric classification of the different series of representations of the group $G=\operatorname{Sl}(2, \mathbb{R})$ (cf. Theorem 4.7 below). 
Acknowledgment. We express our gratitude to Joseph Bernstein for generously sharing his insights with us. We thank the number theorists Erez Lapid and Andre Reznikov for pointing out interesting questions and their useful hints to the literature. We are indebted to Philip Foth who did an elegant matrix computation for us. We thank Joachim Hilgert for asking a good question during one of our presentations of the subject.

Last but not least we acknowledge the screening and comments of two professional referees.

\section{The Complex Crown and its Distinguished Boundary}

This section is divided into two parts. First we recall the definition and basic properties of the complex crown $\Xi$ of a Riemannian symmetric space $X$ (see [KrS2] for a comprehensive account). Second we shall unify and extend results from [GiK1] on the distinguished boundary of $\Xi$.

2.1 The complex crown. Let $G$ be a connected, real semisimple, noncompact Lie group. Write $\mathfrak{g}$ for the Lie algebra of $G$ and denote by $\mathfrak{g}_{\mathbb{C}}$ its complexification. We fix a maximal compact subalgebra $\mathfrak{k} \subset \mathfrak{g}$ and set $K=\exp (\mathfrak{k})$.

Let us denote by $G_{\mathbb{C}}$ the universal complexification of $G$ and by $\iota: G \rightarrow G_{\mathbb{C}}$ the homomorphism sitting over the injection $\mathfrak{g} \hookrightarrow \mathfrak{g}_{\mathbb{C}}$. Write $K_{\mathbb{C}}$ for the analytic subgroup of $G_{\mathbb{C}}$ corresponding to $\mathfrak{k}_{\mathbb{C}}$.

Our concern is with the Riemannian symmetric space $X=G / K$. The complex symmetric space $X_{\mathbb{C}}=G_{\mathbb{C}} / K_{\mathbb{C}}$ naturally acts as a complexification of $X$ and the assignment $g K \mapsto \iota(g) K_{\mathbb{C}}$ identifies $X$ as a totally real submanifold of $X_{\mathbb{C}}$ in a $G$-equivariant way. We denote the base point $e K_{\mathbb{C}}$ of $X_{\mathbb{C}}$ by $x_{0}$.

REMARK 2.1. Let $\mathfrak{g}=\mathfrak{g}_{1}+\ldots+\mathfrak{g}_{l}$ be the factorization of $\mathfrak{g}$ in simple Lie algebras and let $\mathfrak{k}=\mathfrak{k}_{1}+\ldots+\mathfrak{k}_{l}$ be the associated splitting for $\mathfrak{k}$. Denote by $G_{j}, K_{j}$ the analytic subgroups of $G$ corresponding to $\mathfrak{g}_{j}, \mathfrak{k}_{j}$. Then with $X_{j}=G_{j} / K_{j}$ there is the equivariant isomorphism

$$
X \simeq X_{1} \times \ldots \times X_{l} .
$$

In a similar manner (and obvious notation)

$$
X_{\mathbb{C}} \simeq X_{1, \mathbb{C}} \times \ldots \times X_{l, \mathbb{C}} .
$$

In the light of the discussion in Remark 2.1 it is no loss of generality to assume henceforth that $\mathfrak{g}$ is simple.

Let $\mathfrak{g}=\mathfrak{k} \oplus \mathfrak{p}$ be the Cartan decomposition associated to the choice of $\mathfrak{k}$, and choose $\mathfrak{a}$ a maximal abelian subspace in $\mathfrak{p}$. The complex crown $\Xi$ of $X$ 
is by definition

$$
\Xi=G \exp (i \pi \Omega / 2) \cdot x_{0} \subset X_{\mathbb{C}},
$$

where $\Omega \subset \mathfrak{a}$ is given by

$$
\Omega=\{Y \in \mathfrak{a} \mid \operatorname{spec}(\operatorname{ad} Y) \subset]-1,1[\} .
$$

According to [AG], $\Xi$ is a $G$-invariant open subdomain of $X_{\mathbb{C}}$ with the $G$ action proper. Actually $\Xi$ is Stein (see $[\mathrm{KrS} 2]$ and the references therein). Let us point out that $\Xi$ is independent of the choice of the flat $\mathfrak{a}$ and therefore naturally attached to $X$.

The set $\Omega$ can be described in terms of the restricted root system $\Sigma=$ $\Sigma(\mathfrak{g}, \mathfrak{a})$ as follows:

$$
\Omega=\{Y \in \mathfrak{a}|| \alpha(Y) \mid<1 \forall \alpha \in \Sigma\} .
$$

In particular we see that $\bar{\Omega}$ is a compact $W$-invariant polyhedron. Here $W$, as usual, denotes the Weyl group of $\Sigma$.

REMARK 2.2 (Realization in the tangent bundle). Set $\Omega^{K}=\frac{\pi}{2} \operatorname{Ad}(K) \Omega$. As $\Omega$ is an open $W$-invariant convex subset of $\mathfrak{a}$, Kostant's linear convexity theorem implies that $\Omega^{K} \subset \mathfrak{p}$ is an open $K$-invariant convex subset of $\mathfrak{p}$. Write $T X=G \times_{K} \mathfrak{p}$ for the tangent bundle of $X$. Notice that $G$ acts properly on $T X$ and that $G \times{ }_{K} \Omega^{K}$ is a contractible $G$-equivariant subset of $T X$ (base and fiber are contractible). In $[\mathrm{AG}]$ it was shown that the map

$$
G \times{ }_{K} \Omega^{K} \rightarrow \Xi, \quad[g, Y] \mapsto g \exp (i Y) \cdot x_{0}
$$

is a $G$-equivariant diffeomorphism. In particular, $G$ acts properly on $\Xi$ and $\Xi$ is contractible.

In the sequel we write $\mathfrak{t}=i \mathfrak{a}$ and let $T=\exp \mathfrak{t}$ be the corresponding torus in $G_{\mathbb{C}}$. Notice that $T_{\mathbb{C}}=A_{\mathbb{C}}=A T$ with $A=\exp \mathfrak{a}$. We will also use the notation $T_{\Omega}=\exp (i \pi \Omega / 2)$.

REMARK 2.3 (The boundary of $\Xi$ ). (i) (Semisimple boundary part) The topological boundary $\partial \Xi$ is a complicated union of $G$-orbits. This is because not all $G$-orbits in $\partial \Xi$ meet T. $x_{0}$. Those which do make up the semisimple (or elliptic) part of the boundary $\partial_{s} \Xi=G \exp (i \pi \partial \Omega / 2) \cdot x_{0}$ of $\Xi$ (see [AG], $[\mathrm{KrS} 2])$. Equivalently, $\partial_{s} \Xi$ describes the closed $G$-orbits in $\partial \Xi$. One knows that each $G$-orbit in $\partial \Xi$ has a a unique semisimple orbit in its closure [FH], but a satisfactory general description of $\partial \Xi$ is still missing.

(ii) (Properness) The polyhedron $\Omega$ is maximal with regard to proper $G$-action, i.e. there does not exists a larger connected subset $\tilde{\Omega} \supset \Omega$ such that $G$ would act properly on $G \exp (i \pi \tilde{\Omega} / 2) \cdot x_{0}$ (cf. [AG]). We mention that $G$-stabilizers of points in $\exp (i \pi \partial \Omega / 2) \cdot x_{0}$ are noncompact subgroups [AG].

(iii) (Dependence on isogenies) It follows from (2.4) that $\Xi$ is homeomorphic to $\mathfrak{p} \times \Omega^{K}$. It means in particular that $\Xi$ only depends on the 
isogeny class of the connected group $G$. However, the situation becomes different once we start to consider the boundary $\partial \Xi$ of the crown in $X_{\mathbb{C}}$. It turns out that $\partial \Xi$ is sensitive with regard to the choice of the connected group $G$; for instance $\partial \Xi$ is different for $\mathrm{SO}(n, \mathbb{C})$ and its simply connected cover $\mathrm{SO}(n, \mathbb{C})^{\sim}$. We will comment more on that when we will discuss collapsing of boundary orbits below (Example 2.15).

2.2 Distinguished and minuscule boundary of the crown. The distinguished boundary $\partial_{d} \Xi$ of the crown, introduced in [GiK1], is defined by

$$
\partial_{d} \Xi=G \exp \left(i \pi \partial_{e} \Omega / 2\right) \cdot x_{0} \subset \partial \Xi,
$$

where $\partial_{e} \Omega$ is the (finite set) of extreme points of the compact polyhedron $\bar{\Omega}$. In view of this definition and the results of the previous subsection, we may and will assume that $\mathfrak{g}$ is simple in this subsection. Let us recall that the distinguished boundary plays the rôle of a noncompact Shilov-type boundary of $\Xi$; one has the following elementary result.

Proposition 2.4 [GiK1]. Let $f$ be a holomorphic function on $\Xi$ which extends to a bounded continuous function on $\bar{\Xi}$. Then

$$
\sup _{x \in \Xi}(|f(x)|)=\sup _{x \in \partial_{d}(\Xi)}(|f(x)|) .
$$

In [GiK1] a complete characterization of those crowns $\Xi$ was given which admit symmetric spaces as components in $\partial_{d} \Xi$. Cases relevant for [GiK1] are those $\Sigma$ which are not of type $E_{8}, G_{2}$ or $F_{4}$.

The objective of this section is to give a uniform approach to $\partial_{d} \Xi$ in the general case. Our first result is a description of $\partial_{e} \Omega$ in terms of structure theory which is stunningly simple (cf. Theorem 2.6 below). We will define the minuscule part of the distinguished boundary and tie it with the results of [GiK1]. After that we classify the non-symmetric boundary components of $\partial_{d} \Xi$. Finally we discuss collapsing of distinguished boundary orbits.

Write $\Sigma^{l}=\{\alpha: 2 \alpha \notin \Sigma\}$ for the irreducible reduced subsystem of unmultipliable roots in $\Sigma=\Sigma(\mathfrak{g}, \mathfrak{a})$. It is clear that $\Sigma^{l}$ completely describes $\Omega$, i.e.

$$
\Omega=\left\{Y \in \mathfrak{a}|| \alpha(Y) \mid<1, \forall \alpha \in \Sigma^{l}\right\} .
$$

Fix a basis for $\Sigma^{l}$, say $\Pi=\left\{\alpha_{1}, \ldots, \alpha_{n}\right\}$, and write $C \subset \mathfrak{a}$ for the closure of the associated Weyl chamber. Let $\beta$ be the highest root corresponding to $\Pi$ and

$$
\beta=k_{1} \alpha_{1}+\ldots+k_{n} \alpha_{n}
$$

its expansion in the simple roots (hence $k_{i} \in \mathbb{Z}_{>0}$ ). We record the obvious relation

$$
\bar{\Omega} \cap C=\{Y \in C: \beta(Y) \leq 1\} .
$$


It means that $\bar{\Omega} \cap C$ is a fundamental domain for the affine Weyl group $W^{\text {aff }}=W \ltimes Q^{\vee}$ with $Q^{\vee}=\operatorname{span}_{\mathbb{Z}} \Sigma^{\vee}$ the coroot lattice in $\mathfrak{a}$ (observe that $\left.\operatorname{span}_{\mathbb{Z}} \Sigma^{\vee}=\operatorname{span}_{\mathbb{Z}}\left(\Sigma^{l}\right)^{\vee}\right)$.

Define $\omega_{i} \in \mathfrak{a}$ by $\alpha_{j}\left(\omega_{i}\right)=\delta_{i j}$. It is straightforward from (2.7) that

$$
\partial_{e} \Omega \cap C \subset\left\{\omega_{1} / k_{1}, \ldots, \omega_{n} / k_{n}\right\},
$$

and so $\partial_{e} \Omega \subset W .\left\{\omega_{1} / k_{1}, \ldots, \omega_{n} / k_{n}\right\}$ (cf. [GiK1, Lem. 3.17]).

In general, the inclusion in (2.8) is proper and we have to determine which $\omega_{i} / k_{i}$ actually occur. The key observation is contained in Lemma 2.5 below.

We need some terminology. Let $(V,(\cdot, \cdot))$ be an Euclidean space and $W \subset \mathrm{O}(V)$ be a Weyl group of finite type associated to a root system with root basis $F$. We shall assume that the action is effective, or equivalently that $V^{*}=\operatorname{span}_{\mathbb{R}} F$. For a subset $P \subset F$ let $W_{P}<W$ be the corresponding parabolic subgroup. As before

$$
C=\{v \in V: \alpha(v) \geq 0 \forall \alpha \in F\}
$$

denotes the closure of the Weyl chamber. A closed convex cone $\Gamma \subset V$ will be called non-degenerate if its edge $E(\Gamma)=\Gamma \cap-\Gamma$ is equal to $\{0\}$. Clearly $\Gamma$ is non-degenerate iff there exists a linear functional $\omega \in V^{*}$ such that $\left.\omega\right|_{\Gamma \backslash\{0\}}>0$.

Lemma 2.5. Let $W$ be a Weyl group of finite type acting effectively on an Euclidean space $V$. Let $C$ be the closure of the corresponding Weyl chamber. Then the following statements are equivalent:

(i) $W$ is irreducible.

(ii) $W_{P} . C$ is non-degenerate for all proper subsets $P \subsetneq F$.

(iii) $W_{P} . C$ is non-degenerate for a maximal proper subset $P \subsetneq F$.

Proof. (i) $\Rightarrow$ (ii): If $P=\emptyset$, then $W_{P} . C=C$ is non-degenerate. So let us henceforth assume that $P \neq \emptyset$. Denote by $V_{\text {fix }}=\left\{v \in V \mid\left(\forall w \in W_{P}\right) w(v)\right.$ $=v\}$ the space of $W_{P}$-fixed points. Then

$$
V=V_{\text {fix }} \oplus V_{\text {eff }}
$$

with $V_{\text {eff }}=\operatorname{span}_{\mathbb{R}} P=V_{\text {fix }}^{\perp}$ the effective part for the $W_{P}$-action. We note that $C \cap V_{\text {fix }} \neq\{0\}$ and fix a non-zero element $u$ in this intersection.

Assume that $W$ is irreducible. According to [Hum, Ch. IV, Ex. 8], one has $(x, y)>0$ for all $x, y \in C \backslash\{0\}$. In particular if $\omega \in V^{*}$ is defined by $\omega(v):=(u, v)$ then $\left.\omega\right|_{C \backslash\{0\}}>0$. As $u$ is $W_{P}$-fixed, it follows that $\left.\omega\right|_{W_{P} . C \backslash\{0\}}>0$ and consequently $W_{P} . C$ is non-degenerate.

(ii) $\Rightarrow$ (iii) is clear, moving on to (iii) $\Rightarrow$ (i): We argue by contradiction and assume that $W$ is reducible. Then there exist splittings $W=W_{1} \times W_{2}$, 
$V=V_{1} \oplus V_{2}, F=F_{1} \amalg F_{2}$ with $W_{1}$ irreducible, $F_{1} \subset P$ and $V_{1}, V_{2} \neq\{0\}$. But then $V_{1} \subset W_{P} . C$ and $W_{P} . C$ is degenerate.

Let us now return to our initial setting with the irreducible restricted root system $\Sigma=\Sigma(\mathfrak{g}, \mathfrak{a})$ (then the reduced root system $\Sigma^{l}$ of unmultipliable roots is irreducible as well). We write $D$ for the Dynkin diagram associated with the bases $\Pi$ of $\Sigma^{l}$, and $D^{*}=D\left(W^{\text {aff }}\right)$ for its affine extension. Let $\Pi_{0}=\left\{\alpha_{0}, \alpha_{1}, \ldots, \alpha_{n}\right\}$ denote the underlying set of affine simple roots.

Theorem 2.6. Let $\mathfrak{g}$ be a simple Lie algebra and $\Sigma=\Sigma(\mathfrak{g}, \mathfrak{a})$ the associated irreducible root system. Then for all $1 \leq i \leq n$ the following statements are equivalent:

(i) $\omega_{i} / k_{i} \in \partial_{e} \Omega$;

(ii) $D^{*}-\left\{\alpha_{i}\right\}$ is connected.

Proof. Fix $1 \leq i \leq n$ and denote the stabilizer of $\omega_{i} / k_{i}$ in $W^{\text {aff }}$ by $W^{(i)}$. Notice that $W^{(i)} \simeq W\left(D^{*}-\left\{\alpha_{i}\right\}\right)$ is a Weyl group of finite type, and that $\Pi^{(i)}=\left\{\alpha_{0}, \ldots, \alpha_{i-1}, \alpha_{i+1}, \ldots, \alpha_{n}\right\}$ is a set of simple roots for its root system. Let us denote by $C^{(i)}$ the associated closed Weyl chamber.

Let $U$ denote an open ball around $\omega_{i} / k_{i}$ such that for $w \in W$ one has $w(U) \cap C \neq \emptyset$ iff $w$ fixes $\omega_{i} / k_{i}$. The isotropy group of $\omega_{i} / k_{i}$ in $W$ is $W_{P}$ where $P=\Pi \cap \Pi^{(i)}$. Observe that $P$ is a maximal proper subset both of $\Pi$ and of $\Pi^{(i)}$.

Observe that $C \cap \bar{\Omega}$ is the fundamental alcove of $W^{\text {aff }}$ with respect to $\Pi_{0}$. Hence $(C \cap \bar{\Omega}) \cap U=C^{(i)} \cap U$. Moreover $\bar{\Omega} \cap U=W(C \cap \bar{\Omega}) \cap U=$ $W_{P}(C \cap \bar{\Omega}) \cap U=W_{P}\left(C^{(i)} \cap U\right)=W_{P}^{(i)}\left(C^{(i)} \cap U\right)=W_{P}^{(i)} C^{(i)} \cap U$. Hence $\omega_{i} / k_{i}$ is an extremal point of $\bar{\Omega}$ iff the convex cone $W_{P}^{(i)} C^{(i)}$ (with vertex $\left.\omega_{i} / k_{i}\right)$ is non-degenerate. Apply Lemma 2.5.

Let us call $\omega_{i}$ minuscule if $k_{i}=1$. Notice that $\omega_{i} \in \partial \Omega$. Let us denote the union of all $W$-orbits through minuscule $\omega_{i}$ by $\partial_{m} \Omega$ and refer to it as the minuscule part of $\partial \Omega$. Similarly we define the minuscule boundary of $\Xi$ by

$$
\partial_{m} \Xi=G \exp \left(i \pi \partial_{m} \Omega / 2\right) \cdot x_{0} .
$$

Proposition 2.7. One has

$$
\partial_{m} \Omega \subset \partial_{e} \Omega
$$

and in particular $\partial_{m} \Xi \subset \partial_{d} \Xi$.

Proof. Let $A=\left(a_{i j}\right)$ (with $i, j \in\{0,1, \ldots, n\}$ ) be the generalized Cartan matrix associated with the extended Dynkin diagram $D^{*}$. We consider $A$ as a matrix with respect to the bases $\left\{\alpha_{0}, \alpha_{1}, \ldots, \alpha_{n}\right\}$ of affine simple roots. 
By elementary theory of generalized Cartan matrices (cf. [K, Th. 4.8]) the one-dimensional kernel of $A$ is generated by a unique positive, primitive element $\delta$ in the affine root lattice, namely $\delta=\alpha_{0}+\beta$. In other words, if we put $k_{0}=1$ then for each $j, 2 k_{j}+\sum_{i \neq j} a_{j i} k_{i}=0$. Hence if $\omega_{j}$ is minuscule (i.e. $k_{j}=1$ ) then either $\alpha_{j}$ is an end point in $D^{*}$ (i.e. has only one neighbor in $D^{*}$ ) or else $\alpha_{j}$ has precisely two neighbors $\alpha_{i}, \alpha_{l}$ with $k_{i}=k_{l}=1$. But in this last case $D^{*}$ must be (by an easy inductive argument) a circular graph. We conclude in both cases that $D^{*}-\left\{\alpha_{j}\right\}$ is connected as desired.

For later reference and convenience to the reader we list $\partial_{e} \Omega$ and $\partial_{m} \Omega$. Theorem 2.6 and the tables of [Bou] yield:

\begin{tabular}{|c|c|c|}
\hline \multicolumn{3}{|c|}{ Distinguished and minuscule boundary of $\Omega$} \\
\hline \hline$\Sigma$ & $\partial_{e} \Omega \cap C$ & $\partial_{m} \Omega \cap C$ \\
\hline \hline$A_{n}$ & $\omega_{1}, \ldots, \omega_{n}$ & $\omega_{1}, \ldots, \omega_{n}$ \\
\hline$B_{n}(n \geq 3)$ & $\omega_{1}, \omega_{n} / 2$ & $\omega_{1}$ \\
\hline$C_{n}, B C_{n}$ & $\omega_{n}$ & $\omega_{n}$ \\
\hline$D_{n}(n \geq 4)$ & $\omega_{1}, \omega_{n-1}, \omega_{n}$ & $\omega_{1}, \omega_{n-1}, \omega_{n}$ \\
\hline$E_{6}$ & $\omega_{1}, \omega_{6}$ & $\omega_{1}, \omega_{6}$ \\
\hline$E_{7}$ & $\omega_{2} / 2, \omega_{7}$ & $\omega_{7}$ \\
\hline$E_{8}$ & $\omega_{1} / 2, \omega_{2} / 3$ & $\emptyset$ \\
\hline$F_{4}$ & $\omega_{4} / 2$ & $\emptyset$ \\
\hline$G_{2}$ & $\omega_{1} / 3$ & $\emptyset$ \\
\hline \hline
\end{tabular}

Table 1

REMARK 2.8 (Correcting literature). The first named author would like to take the opportunity to point out an error in [GiK1] regarding $\partial_{e} \Omega$ for the $E_{7}$-case. Due to a computational mistake the $W$-orbit through $\omega_{2} / 2$ was missed.

For a point $\omega_{j} / k_{j} \in \partial \Omega$ set

$$
z_{j}=\exp \left(i \pi \omega_{j} / 2 k_{j}\right) \cdot x_{0} \in \partial_{d} \Xi
$$

and denote by $H_{j}$ the stabilizer of $G$ in $z_{j}$. We remarked earlier that $H_{j}$ is a noncompact subgroup. Let us denote by $\mathfrak{h}_{j}$ its Lie algebra. Our next objective is to classify the stabilizer algebras $\mathfrak{h}_{j}$ for those $z_{j}$ which appear in $\partial_{d} \Xi$.

Write $F=A_{\mathbb{C}} \cap K_{\mathbb{C}}=T \cap K$ and notice that $F$ is a finite two group. We will often identify $A_{\mathbb{C}} \cdot x_{0}$ with $A_{\mathbb{C}} / F$ and remark that elements $z \in A_{\mathbb{C}} \cdot x_{0}$ have well-defined squares $z^{2} \in A_{\mathbb{C}}$. For each $1 \leq j \leq n$ let us define the 
centralizer subgroup

$$
G_{j}:=Z\left(z_{j}^{4}\right)=\left\{g \in G \mid z_{j}^{4} g z_{j}^{-4}=g\right\},
$$

and denote by $\mathfrak{g}_{j}$ its Lie algebra.

Lemma 2.9. Let $1 \leq j \leq n$. Then the following assertions hold:

(i) $\mathfrak{g}_{j}=\mathfrak{g}$ if and only if $\omega_{j}$ is minuscule.

(ii) $\mathfrak{g}_{j}$ is a 3-graded reductive Lie algebra

$$
\mathfrak{g}_{j}=\mathfrak{g}_{j,-} \oplus \mathfrak{g}_{j, 0} \oplus \mathfrak{g}_{j,+}
$$

where $\mathfrak{g}_{j, \pm}=\left\{Y \in \mathfrak{g} \mid\left[\omega_{j}, Y\right]= \pm k_{j} Y\right\}$ and $\mathfrak{g}_{j, 0}=\left\{Y \in \mathfrak{g} \mid\left[\omega_{j}, Y\right]=0\right\}$.

Proof. Associated to $\omega_{j}$ is the standard grading

$$
\mathfrak{g}=\sum_{l=-k_{j}}^{k_{j}} \mathfrak{g}_{l}
$$

with $\mathfrak{g}_{l}=\left\{Y \in \mathfrak{g} \mid\left[\omega_{j}, Y\right]=l Y\right\}$. Notice that $\operatorname{Ad}\left(z_{j}^{4}\right)$ acts on $\mathfrak{g}_{l}$ as the scalar $e^{2 i l \pi / k_{j}}$. The assertions of the lemma follow with $\mathfrak{g}_{j, \pm}=\mathfrak{g}_{ \pm k_{j}}$.

Let us denote by $\theta$ the Cartan involution of $\mathfrak{g}=\mathfrak{k} \oplus \mathfrak{p}$. Observe that $Y \in \mathfrak{g}$ belongs to $\mathfrak{h}_{j}$ if and only if $\operatorname{Ad}\left(z_{j}^{-1}\right)(Y) \in \mathfrak{k}_{\mathbb{C}} ;$ in other words

$$
\mathfrak{h}_{j}=\left\{Y \in \mathfrak{g} \mid \operatorname{Ad}\left(z_{j}^{2}\right)(\theta Y)=Y\right\}
$$

(cf. [GiK1, Lem. 3.4]). We reveal the structure of $\mathfrak{h}_{j}$.

Lemma 2.10. Let $1 \leq j \leq n$. Then $\mathfrak{h}_{j}$ is $\theta$-stable subalgebra of $\mathfrak{g}_{j}$. Moreover, its Cartan decomposition is given by

$$
\mathfrak{h}_{j}=\mathfrak{g}_{0, j}^{\theta} \oplus\left(\mathfrak{g}_{j,-} \oplus \mathfrak{g}_{j,+}\right)^{-\theta} .
$$

Proof. Recall the grading $\mathfrak{g}=\sum_{l=-k_{j}}^{k_{j}} \mathfrak{g}_{l}$ from (2.12). Then for each $0 \leq$ $l \leq k_{j}$ the operator $\operatorname{Ad}\left(z_{j}^{2}\right) \circ \theta$ leaves $\left(\mathfrak{g}_{l} \oplus \mathfrak{g}_{-l}\right)_{\mathbb{C}}$ stable; explicitly

$$
\left(Y_{l}, Y_{-l}\right) \mapsto\left(e^{i l \pi / k_{j}} \theta\left(Y_{-l}\right), e^{-i l \pi / k_{j}} \theta\left(Y_{l}\right)\right) \quad\left(Y_{l}, Y_{-l}\right) \in\left(\mathfrak{g}_{l} \oplus \mathfrak{g}_{-l}\right)_{\mathbb{C}} .
$$

Hence $\left(\operatorname{Ad}\left(z_{j}^{2}\right) \circ \theta\right)\left(\mathfrak{g}_{l} \oplus \mathfrak{g}_{-l}\right) \cap \mathfrak{g} \neq\{0\}$ precisely for $l=0, k_{j}$. The assertions of the lemma follow.

Corollary 2.11. Let $1 \leq j \leq n$. Then $\operatorname{dim} \mathfrak{h}_{j} \leq \operatorname{dim} \mathfrak{k}$ with equality precisely if $\omega_{j}$ is minuscule.

As a consequence of Lemma 2.9 and Lemma 2.10 we can extend [GiK1, Th. 3.26(2)].

Theorem 2.12. For a boundary orbit $G . z_{j} \subset \partial \Xi$ the following statements are equivalent:

(i) $\omega_{j}$ is minuscule;

(ii) $\operatorname{dim} \mathfrak{h}_{j}=\operatorname{dim} \mathfrak{k}$; 
(iii) $\operatorname{Ad}\left(z_{j}^{-1}\right)\left(\mathfrak{h}_{j}\right)_{\mathbb{C}}=\mathfrak{k}_{\mathbb{C}}$;

(iv) $\mathfrak{h}_{j}$ is a symmetric subalgebra of $\mathfrak{g}$;

(v) $G . z_{j}$ is a totally real submanifold of $X_{\mathbb{C}}$;

(vi) $G . z_{j}$ is a totally real submanifold of $X_{\mathbb{C}}$ of maximal dimension.

Proof. (i) $\Longleftrightarrow$ (ii): Corollary 2.11.

(ii) $\Longleftrightarrow$ (iii): $\operatorname{Ad}\left(z_{j}^{-1}\right) \mathfrak{h}_{j} \subset \mathfrak{k}_{\mathbb{C}}$ holds for all $1 \leq j \leq n$ by the definition of $\mathfrak{h}_{j}$.

(i) $\Leftarrow$ (iv): If $\omega_{j}$ is minuscule, then $\mathfrak{g}_{j}=\mathfrak{g}$ by Lemma 2.9(i). In particular $\tau_{j}=\operatorname{Ad}\left(z_{j}^{2}\right) \circ \theta$ defines an involution and $\mathfrak{h}_{j}$ being the $\tau_{j}$-fixed point set is symmetric.

(iv) $\Rightarrow$ (i): Notice that $\mathfrak{g}_{j}$ is a reductive subalgebra properly containing $\mathfrak{h}_{j}$. Now if $\mathfrak{h}_{j}$ is symmetric, then it is a maximally reductive proper subalgebra of $\mathfrak{g}$. Thus $\mathfrak{g}=\mathfrak{g}_{j}$, i.e. $\omega_{j}$ is minuscule.

(v) $\Rightarrow$ (ii): If $G . z_{j}$ is totally real, then $\operatorname{dim}_{\mathbb{R}} G \cdot z_{j} \leq \operatorname{dim}_{\mathbb{R}} X$. The latter inequality rewrites as $\operatorname{dim} \mathfrak{h}_{j} \geq \operatorname{dim} \mathfrak{k}$. Because of $\operatorname{dim} \mathfrak{h}_{j} \leq \operatorname{dim} \mathfrak{k}$ in all cases, it follows that $\operatorname{dim} \mathfrak{h}_{j}=\operatorname{dim} \mathfrak{k}$.

(vi) $\Rightarrow(v)$ is clear.

(ii) $\Rightarrow$ (vi): (ii) implies that $\operatorname{dim}_{\mathbb{R}} G \cdot z_{j}=\operatorname{dim}_{\mathbb{R}} X$. It remains to show that $G . z_{j}$ is totally real. By $G$-homogeneity, it is sufficient to show that $T_{z_{j}}\left(G . z_{j}\right)$ is totally real in $T_{z_{j}}\left(X_{\mathbb{C}}\right)$. The assignment $\left.Y \mapsto \frac{d}{d t}\right|_{t=0} \exp (t Y) \cdot z_{j}$ identifies $\mathfrak{g}_{\mathbb{C}} / \operatorname{Ad}\left(z_{j}\right) \mathfrak{k}_{\mathbb{C}}$ with $T_{z_{j}}\left(X_{\mathbb{C}}\right)$. Now observe that $\mathfrak{g}_{\mathbb{C}} / \operatorname{Ad}\left(z_{j}\right) \mathfrak{k}_{\mathbb{C}}=$ $\mathfrak{g}_{\mathbb{C}} / \mathfrak{h}_{\mathbb{C}}$ by the equivalence of (ii) and (iii). Thus all we have to show is that $\mathfrak{g}+\mathfrak{h}_{\mathbb{C}} / \mathfrak{h}_{\mathbb{C}}$ is totally real in $\mathfrak{g}_{\mathbb{C}} / \mathfrak{h}_{\mathbb{C}}$, which is apparent.

Remark 2.13. Suppose that $\omega_{j}$ is minuscule. Then $\mathfrak{g}=\mathfrak{g}_{j,-} \oplus \mathfrak{g}_{j, 0} \oplus \mathfrak{g}_{j,+}$ is a 3-graduation and $\tau_{j}=\operatorname{Ad}\left(z_{j}^{2}\right) \circ \theta$ is an involution with fixed-point algebra $\mathfrak{h}_{j}$. In other words $\left(\mathfrak{g}, \mathfrak{h}_{j}\right)$ is a noncompactly causal (NCC) symmetric pair. Moreover all (NCC) symmetric pairs arise in this fashion. For a proof of all this we refer to the paper [Ka2] of Professor Soji Kaneyuki (specifically Th. 3.1).

For the concrete classification of the $\mathfrak{h}_{j}$ in the minuscule case we allow ourselves to refer alternatively to [GiK1, Th. 3.25].

We wish to complete the classification of $\partial_{d} \Xi$ by listing all the nonminuscule cases. The most degenerate situation might deserve special attention.

EXAMPLE 2.14 (The distinguished boundary of $G_{2}$ ). Let us consider the case of $\mathfrak{g}=G_{2}$. We use the terminology of [Bou]. With $\Pi=\left\{\alpha_{1}, \alpha_{2}\right\}$ the positive roots list as

$$
\alpha_{1}, \alpha_{2}, \alpha_{1}+\alpha_{2}, 2 \alpha_{1}+\alpha_{2}, 3 \alpha_{1}+\alpha_{2}, 3 \alpha_{1}+2 \alpha_{2} .
$$


We have $\partial_{e} \Omega=W \cdot \omega_{1} / 3$. Hence

$$
\begin{aligned}
& \mathfrak{g}_{1,0}=\mathfrak{a} \oplus \mathfrak{g}^{\alpha_{2}} \oplus \mathfrak{g}^{-\alpha_{2}} \simeq \mathfrak{s l}(2, \mathbb{R}) \times \mathbb{R} \\
& \mathfrak{g}_{1,1}=\mathfrak{g}^{3 \alpha_{1}+\alpha_{2}} \oplus \mathfrak{g}^{3 \alpha_{1}+2 \alpha_{2}} \simeq \mathbb{R}^{2}, \\
& \mathfrak{g}_{1,-1}=\mathfrak{g}^{-3 \alpha_{1}-\alpha_{2}} \oplus \mathfrak{g}^{-3 \alpha_{1}-2 \alpha_{2}} \simeq \mathbb{R}^{2},
\end{aligned}
$$

and so $\mathfrak{g}_{1} \simeq \mathfrak{s l}(3, \mathbb{R})$. Finally Lemma 2.10 implies $\mathfrak{h}_{1} \simeq \mathfrak{s l}(2, \mathbb{R})$.

Let $z_{j}$ be a non-minuscule boundary points. A glance at Table 1 above shows that $k_{j}=2$ except for $G_{2}$ and one case in $E_{8}$. Thus $\mathfrak{g}=\sum_{j=-k_{j}}^{k_{j}} \mathfrak{g}_{j}$ is a 5 -grading for most of the cases.

Combining Lemma 2.10 and Lemma 2.9 with Kaneyuki's classification of the even part of 5 -graded Lie algebras [Ka1] we arrive at the following two lists. For the exceptional cases we use [Ka1, Tab. I, II].

\begin{tabular}{|c|c|c|c|c|c|}
\hline \multicolumn{7}{|c|}{ Exceptional non-minuscule cases } \\
\hline \hline $\mathfrak{g}$ & $\Sigma$ & $j$ & $\mathfrak{g}_{0, j}$ & $\mathfrak{g}_{j}$ & $\mathfrak{h}_{j}$ \\
\hline \hline$E_{6(2)}$ & $F_{4}$ & 4 & $\mathfrak{s o}(3,5) \times \mathbb{R} \times i \mathbb{R}$ & $\mathfrak{s o}(4,6) \times i \mathbb{R}$ & $\mathfrak{s o}(1,3) \times \mathfrak{s o}(1,5) \times i \mathbb{R}$ \\
\hline$E_{6(-14)}$ & $B C_{2}$ & 2 & $\mathfrak{s o}(1,7) \times \mathbb{R} \times i \mathbb{R}$ & $\mathfrak{s o}(2,8) \times i \mathbb{R}$ & $\mathfrak{s o}(1,7) \times \mathbb{R} \times i \mathbb{R}$ \\
\hline$E_{7(7)}$ & $E_{7}$ & 2 & $\mathfrak{s l}(7, \mathbb{R}) \times \mathbb{R}$ & $\mathfrak{s l}(8, \mathbb{R})$ & $\mathfrak{s o}(1,7)$ \\
\hline$E_{7(-5)}$ & $F_{4}$ & 4 & $\mathfrak{s o}(3,7) \times \mathfrak{s u}(2) \times \mathbb{R}$ & $\mathfrak{s o}(4,8) \times \mathfrak{s u}(2)$ & $\mathfrak{s o}(1,3) \times \mathfrak{s o}(1,7) \times \mathfrak{s u}(2)$ \\
\hline$E_{8(8)}$ & $E_{8}$ & 1 & $\mathfrak{s o}(7,7) \times \mathbb{R}$ & $\mathfrak{s o}(8,8)$ & $\mathfrak{s o}(1,7) \times \mathfrak{s o}(1,7)$ \\
\hline$E_{8(8)}$ & $E_{8}$ & 2 & $\mathfrak{s l}(8, \mathbb{R}) \times \mathbb{R}$ & $\mathfrak{s l}(9, \mathbb{R})$ & $\mathfrak{s o}(1,8)$ \\
\hline$E_{8(-24)}$ & $F_{4}$ & 4 & $\mathfrak{s o}(3,11) \times \mathbb{R}$ & $\mathfrak{s o}(4,12)$ & $\mathfrak{s o}(1,3) \times \mathfrak{s o}(1,11)$ \\
\hline$F_{4(4)}$ & $F_{4}$ & 4 & $\mathfrak{s o}(3,4) \times \mathbb{R}$ & $\mathfrak{s o}(4,5)$ & $\mathfrak{s o}(1,3) \times \mathfrak{s o}(1,4)$ \\
\hline$F_{4(-20)}$ & $B C_{1}$ & 1 & $\mathfrak{s o}(7) \times \mathbb{R}$ & $\mathfrak{s o}(1,8)$ & $\mathfrak{s o}(1,7)$ \\
\hline$G_{2}$ & $G_{2}$ & 1 & $\mathfrak{s l}(2, \mathbb{R}) \times \mathbb{R}$ & $\mathfrak{s l}(3, \mathbb{R})$ & $\mathfrak{s o}(1,2)$ \\
\hline$E_{7}^{\complement}$ & $E_{7}$ & 2 & $\mathfrak{s l}(7, \mathbb{C}) \times \mathbb{C}$ & $\mathfrak{s l}(8, \mathbb{C})$ & $\mathfrak{s u}(1,7)$ \\
\hline$E_{8}^{\complement}$ & $E_{8}$ & 1 & $\mathfrak{s o}(14, \mathbb{C}) \times \mathbb{C}$ & $\mathfrak{s o}(16, \mathbb{C})$ & $\mathfrak{s o}(2,14)$ \\
\hline$E_{8}^{\complement}$ & $E_{8}$ & 2 & $\mathfrak{s l}(8, \mathbb{C}) \times \mathbb{C}$ & $\mathfrak{s l}(9, \mathbb{C})$ & $\mathfrak{s u}(1,8)$ \\
\hline$F_{4}^{\complement}$ & $F_{4}$ & 4 & $\mathfrak{s o}(7, \mathbb{C}) \times \mathbb{C}$ & $\mathfrak{s o}(9, \mathbb{C})$ & $\mathfrak{s o}(2,7)$ \\
\hline$G_{2}^{\complement}$ & $G_{2}$ & 1 & $\mathfrak{s l}(2, \mathbb{C}) \times \mathbb{C}$ & $\mathfrak{s l}(3, \mathbb{C})$ & $\mathfrak{s u}(1,2)$ \\
\hline \hline
\end{tabular}

Table 2

For the classical cases we apply [Ka1, Th.3.2], and note that the first two cases below were already contained in [GiK1, Th. 3.25].

We conclude this section with a discussion of collapsing of boundary orbits. Let $z_{j}, z_{l} \in \partial_{d} \Xi$ with $j \neq l$. If $G_{\mathbb{C}}$ is simply connected and $G \subset G_{\mathbb{C}}$, then $G . z_{j} \neq G . z_{l}$, i.e. G. $z_{j} \cap G . z_{l}=\emptyset$ (cf. [GiK1, Th. 3.6]). In the general case it might happen that $G . z_{j}=G . z_{k}$ and we say that $\omega_{j} / k_{j}$ and $\omega_{l} / k_{l}$ collapse in $\partial_{d} \Xi$. Collapsing appears when there exist outer automorphisms. We refrain from complete results but would like to mention some important examples. 


\begin{tabular}{|c|c|c|c|c|}
\hline \multicolumn{7}{|c|}{ Classical non-minuscule cases } \\
\hline \hline $\mathfrak{g}$ & $\Sigma$ & $j$ & $\mathfrak{g}_{j}$ & $\mathfrak{h}_{j}$ \\
\hline \hline $\mathfrak{s o}(p, q)(3 \leq p<q)$ & $B_{p}$ & $p$ & $\mathfrak{s o}(p, p) \times \mathfrak{s o}(q-p)$ & $\mathfrak{s o}(p, \mathbb{C}) \times \mathfrak{s o}(q-p)$ \\
\hline $\mathfrak{s o}(2 n+1, \mathbb{C})(n \geq 3)$ & $B_{n}$ & $n$ & $\mathfrak{s o}(2 n, \mathbb{C})$ & $\mathfrak{s o}(2 n)$ \\
\hline $\mathfrak{s u}(p, q)(p<q)$ & $B C_{p}$ & $p$ & $\mathfrak{s u}(p, p) \times \mathfrak{s u}(q-p)$ & $\mathfrak{s l}(p, \mathbb{C}) \times \mathbb{R} \times \mathfrak{s u}(q-p)$ \\
\hline $\mathfrak{s p}(p, q)(p<q)$ & $B C_{p}$ & $p$ & $\mathfrak{s p}(p, p) \times \mathfrak{s p}(q-p)$ & $\mathfrak{s l}(p, \mathbb{H}) \times \mathbb{R} \times \mathfrak{s p}(q-p)$ \\
\hline $\mathfrak{s o}^{*}(2 n)(n \geq 5$, odd $)$ & $B C_{[n / 2]}$ & {$[n / 2]$} & $\mathfrak{s o}^{*}(2 n-2)$ & $\mathfrak{s l}((n-1) / 2, \mathbb{H}) \times \mathbb{R}$ \\
\hline \hline
\end{tabular}

Table 3

ExAmPle 2.15. (a) Let $G=\operatorname{PSl}(n, \mathbb{K})$ for $\mathbb{K}=\mathbb{R}, \mathbb{C}, \mathbb{H}$. Then $\omega_{j}$ and $\omega_{l}$ collapse precisely for $j+l=n$.

(b) Let $G=\mathrm{SO}(n, n)$ for $n \geq 4$. Then $\omega_{n-1}$ and $\omega_{n}$ collapse.

\section{New Features of $G=\operatorname{Sl}(2, \mathbb{R})$}

This section is devoted to the crown domain associated to the basic group $G=\operatorname{Sl}(2, \mathbb{R})$. It is divided into two parts. In the first half we give a description of the full boundary $\partial \Xi$ as a cone bundle over the affine symmetric space $G / H=\mathrm{Sl}(2, \mathbb{R}) / \mathrm{SO}(1,1)$. In the second part we give a novel description of the crown as a union of unipotent $G$-orbits. Later, via appropriate $\mathrm{Sl}(2, \mathbb{R})$-reduction, we will use the material collected there for our discussion of cusp forms and proper action.

3.1 Corner view. We change perspective. Instead of regarding the crown from the base point $x_{0}$ as a thickening of $X$, we may view $\Xi$ from a corner point $z_{j}$ as a domain bordered by the homogeneous space $G / H_{j}$. The advantage of this perspective is that it leads to a simple characterization of the full boundary $\partial \Xi$ of $\Xi$.

We will give a detailed discussion of the boundary of the complex crown when $G=\operatorname{Sl}(2, \mathbb{R})$. As $\Xi$ is attached to $X$, and so independent of the specific global structure of $G$, we may replace $\mathrm{Sl}(2, \mathbb{R})$ by $G=\mathrm{SO}_{e}(1,2)$. We regard $K=\mathrm{SO}(2, \mathbb{R})$ as a maximal compact subgroup of $G$ under the standard lower-right corner embedding.

Let us define a quadratic form $Q$ on $\mathbb{C}^{3}$ by

$$
Q(\mathbf{z})=z_{0}^{2}-z_{1}^{2}-z_{2}^{2}, \quad \mathbf{z}=\left(z_{0}, z_{1}, z_{2}\right)^{T} \in \mathbb{C}^{3} .
$$

With $Q$ we declare real and complex hyperboloids by

$$
X=\left\{\mathbf{x}=\left(x_{0}, x_{1}, x_{2}\right)^{T} \in \mathbb{R}^{3} \mid Q(\mathbf{x})=1, x_{0}>0\right\}
$$


and

$$
X_{\mathbb{C}}=\left\{\mathbf{z}=\left(z_{0}, z_{1}, z_{2}\right)^{T} \in \mathbb{C}^{3} \mid Q(\mathbf{z})=1\right\} .
$$

We notice that mapping

$$
G_{\mathbb{C}} / K_{\mathbb{C}} \rightarrow X_{\mathbb{C}}, \quad g K_{\mathbb{C}} \mapsto g \cdot \mathbf{x}_{0} \quad\left(\mathbf{x}_{0}=(1,0,0)\right)
$$

is diffeomorphic and that $X$ is identified with $G / K$.

At this point it is useful to introduce coordinates on $\mathfrak{g}=\mathfrak{s o}(1,2)$. We set

$$
\mathbf{e}_{1}=\left(\begin{array}{lll}
0 & 0 & 1 \\
0 & 0 & 0 \\
1 & 0 & 0
\end{array}\right), \quad \mathbf{e}_{2}=\left(\begin{array}{lll}
0 & 1 & 0 \\
1 & 0 & 0 \\
0 & 0 & 0
\end{array}\right), \quad \mathbf{e}_{3}=\left(\begin{array}{ccc}
0 & 0 & 0 \\
0 & 0 & 1 \\
0 & -1 & 0
\end{array}\right)
$$

We notice that $\mathfrak{k}=\mathbb{R} \mathbf{e}_{3}, \mathfrak{p}=\mathbb{R} \mathbf{e}_{1} \oplus \mathbb{R} \mathbf{e}_{2}$ and make our choice of the flat piece $\mathfrak{a}=\mathbb{R} \mathbf{e}_{\mathbf{1}}$. Then $\Omega=(-1,1) \mathbf{e}_{\mathbf{1}}, \Xi=G \exp \left(i(-\pi / 2, \pi / 2) \mathbf{e}_{\mathbf{1}}\right) \cdot \mathbf{x}_{\mathbf{0}}$ and we obtain Gindikin's favorite model of the crown

$$
\Xi=\left\{\mathbf{z}=\mathbf{x}+i \mathbf{y} \in X_{\mathbb{C}} \mid x_{0}>0, Q(\mathbf{x})>0\right\} .
$$

It follows that the boundary of $\Xi$ is given by

$$
\partial \Xi=\partial_{s} \Xi \amalg \partial_{n} \Xi,
$$

with semisimple part

$$
\partial_{s} \Xi=\left\{i \mathbf{y} \in i \mathbb{R}^{3} \mid Q(\mathbf{y})=-1\right\}
$$

and nilpotent part

$$
\partial_{n} \Xi=\left\{\mathbf{z}=\mathbf{x}+i \mathbf{y} \in X_{\mathbb{C}} \mid x_{0}>0, Q(\mathbf{x})=0\right\} .
$$

Notice that $\mathbf{z}_{\mathbf{1}}=\exp \left(i \pi / 2 \mathbf{e}_{\mathbf{1}}\right) \cdot \mathbf{x}_{\mathbf{0}}=(0,0, i)^{T}$ and that the stabilizer of $\mathbf{z}_{1}$ in $G$ is the symmetric subgroup $H=\mathrm{SO}_{e}(1,1)$, sitting inside of $G$ as the upper-left corner block. Hence

$$
\partial_{s} \Xi=\partial_{d} \Xi=G \cdot \mathbf{z}_{\mathbf{1}} \simeq G / H .
$$

Write $\tau$ for the involution on $G$ with fixed point set $H$ and let $\mathfrak{g}=\mathfrak{h} \oplus \mathfrak{q}$ the corresponding $\tau$-eigenspace decomposition. Clearly, $\mathfrak{h}=\mathbb{R} \mathbf{e}_{2}$ and $\mathfrak{q}=$ $\mathfrak{a} \oplus \mathfrak{k}=\mathbb{R} \mathbf{e}_{\mathbf{1}} \oplus \mathbb{R} \mathbf{e}_{\mathbf{3}}$. Notice that $\mathfrak{q}$ breaks as an $\mathfrak{h}$-module into two pieces

$$
\mathfrak{q}=\mathfrak{q}^{+} \oplus \mathfrak{q}^{-}
$$

with

$$
\mathfrak{q}^{ \pm}=\left\{Y \in \mathfrak{q} \mid\left[e_{2}, Y\right]= \pm Y\right\}=\mathbb{R}\left(\mathbf{e}_{1} \pm \mathbf{e}_{2}\right) .
$$

Let us define the $H$-stable pair of half lines

$$
\mathcal{C}=\mathbb{R}_{\geq 0}\left(\mathbf{e}_{\mathbf{1}} \oplus \mathbf{e}_{\mathbf{3}}\right) \cup \mathbb{R}_{\geq 0}\left(\mathbf{e}_{\mathbf{1}}-\mathbf{e}_{\mathbf{3}}\right)
$$

in $\mathfrak{q}=\mathfrak{q}^{+} \oplus \mathfrak{q}^{-}$. We remark that $\mathcal{C}$ is the boundary of the $H$-invariant open cone

$$
\mathcal{W}=\operatorname{Ad}(H)\left(\mathbb{R}_{>0} \mathbf{e}_{\mathbf{1}}\right)=\mathbb{R}_{>0}\left(\mathbf{e}_{\mathbf{1}}+\mathbf{e}_{\mathbf{3}}\right) \oplus \mathbb{R}_{>0}\left(\mathbf{e}_{\mathbf{1}}-\mathbf{e}_{\mathbf{3}}\right) .
$$

Recall that the tangent bundle $T(G / H)$ naturally identifies with $G \times_{H} \mathfrak{q}$ and let us mention that $G \times{ }_{H} \mathcal{C}$ is a $G$-invariant subset thereof. 
Theorem 3.1. For $G=\mathrm{SO}_{e}(1,2)$, the mapping

$$
b: G \times{ }_{H} \mathcal{C} \rightarrow \partial \Xi, \quad[g, Y] \mapsto g \exp (-i Y) . \mathbf{z}_{1}
$$

is a $G$-equivariant homeomorphism.

Proof. It is of course clear that the map is equivariant and continuous. We move to surjectivity. For $s \in \mathbb{R}$,

$$
\begin{aligned}
\exp \left(i s\left(\mathbf{e}_{\mathbf{1}}+\mathbf{e}_{\mathbf{3}}\right)\right) & =\left(\begin{array}{ccc}
1-s^{2} / 2 & s^{2} / 2 & i s \\
-s^{2} / 2 & 1+s^{2} / 2 & i s \\
i s & -i s & 1
\end{array}\right), \\
\exp \left(i s\left(\mathbf{e}_{\mathbf{1}}-\mathbf{e}_{3}\right)\right) & =\left(\begin{array}{ccc}
1-s^{2} / 2 & -s^{2} / 2 & i s \\
s^{2} / 2 & 1+s^{2} / 2 & -i s \\
i s & i s & 1
\end{array}\right) .
\end{aligned}
$$

Therefore,

$$
b\left(\left[\mathbf{1}, s\left(\mathbf{e}_{\mathbf{1}} \pm \mathbf{e}_{\mathbf{3}}\right)\right]\right)=\exp \left(-i s\left(\mathbf{e}_{\mathbf{1}} \pm \mathbf{e}_{\mathbf{3}}\right)\right) \cdot \mathbf{z}_{\mathbf{1}}=(s, \pm s, i)^{T} .
$$

From (3.1)-(3.3),

$$
\partial \Xi=G \cdot\left\{(s, \pm s, i)^{T} \mid s \geq 0\right\}
$$

and surjectivity is forced by (3.5) and $G$-equivariance.

Next, we prove that $b$ is one-to-one. By $G$-equivariance, all we have to show is that

$$
b\left(\left[g, s\left(\mathbf{e}_{\mathbf{1}} \pm \mathbf{e}_{\mathbf{3}}\right)\right]\right)=b\left(\left[\mathbf{1}, t\left(\mathbf{e}_{\mathbf{1}} \pm \mathbf{e}_{\mathbf{3}}\right)\right]\right),
$$

for some $g \in G$ and $s, t \geq 0$, forces $g \in H$ and $\operatorname{Ad}(g)\left(s\left(\mathbf{e}_{\mathbf{1}} \pm \mathbf{e}_{\mathbf{3}}\right)\right)=t\left(\mathbf{e}_{\mathbf{1}} \pm \mathbf{e}_{\mathbf{3}}\right)$. We write (3.7) out and see

$$
g \cdot(s, \pm s, i)^{T}=(t, \pm t, i)^{T} .
$$

We take imaginary parts of this identity and deduce that $g(0,0, i)^{T}=$ $(0,0, i)^{T}=\mathbf{z}_{\mathbf{1}}$, i.e. $g \in H$. With this information we go back in (3.8), take the real part and get $g(s, \pm s, 0)^{T}=(t, \pm t, 0)^{T}$. We observe that the latter means $\operatorname{Ad}(g)\left(s\left(\mathbf{e}_{\mathbf{1}} \pm \mathbf{e}_{\mathbf{3}}\right)\right)=t\left(\mathbf{e}_{\mathbf{1}} \pm \mathbf{e}_{\mathbf{3}}\right)$ and end the proof of injectivity.

Finally we mention that $b$ is an open mapping and this finishes the proof.

Corollary 3.2. For $G=\mathrm{SO}_{e}(1,2)$ one has

$$
\pi_{1}(\partial \Xi)=\pi_{1}(G / H)=\mathbb{Z} .
$$

3.2 Unipotent parameterization. We give now a novel description of the crown as a union of unipotent $G$-orbits.

If not stated otherwise, $G=\operatorname{Sl}(2, \mathbb{R})$. The standard choices of coordinates are

$$
\mathfrak{a}=\mathbb{R}\left(\begin{array}{cc}
1 & 0 \\
0 & -1
\end{array}\right) \quad \text { and } \quad \mathfrak{n}=\mathbb{R}\left(\begin{array}{ll}
0 & 1 \\
0 & 0
\end{array}\right),
$$

and we observe that $\Omega=(-1 / 2,1 / 2)\left(\begin{array}{cc}1 & 0 \\ 0 & -1\end{array}\right)$. 
The key observation is contained in the following lemma.

Lemma 3.3. Let $G=\operatorname{Sl}(2, \mathbb{R})$. For all $0 \leq|t|<\pi / 4, t \in \mathbb{R}$, one has the identity

$$
G\left(\begin{array}{cc}
1 & i \sin 2 t \\
0 & 1
\end{array}\right) \cdot x_{0}=G\left(\begin{array}{cc}
e^{i t} & 0 \\
0 & e^{-i t}
\end{array}\right) \cdot x_{0} \cdot
$$

Proof. For the proof it is convenient to switch to the hyperbolic model and replace $G$ by $\mathrm{SO}_{e}(1,2)$ (we recall that $X=G / K$ and $\Xi$ are independent of the globalization $G$ of $\mathfrak{g}$; Remark 2.3(c)). As before, we choose $\mathfrak{a}=\mathbb{R} \mathbf{e}_{\mathbf{1}}$. We come to our choice of $\mathfrak{n}$. For $z \in \mathbb{C}$ let

and

$$
n_{z}=\left(\begin{array}{ccc}
1+\frac{1}{2} z^{2} & z & -\frac{1}{2} z^{2} \\
z & 1 & -z \\
\frac{1}{2} z^{2} & z & 1-\frac{1}{2} z^{2}
\end{array}\right)
$$

$$
N_{\mathbb{C}}=\left\{n_{z} \mid z \in \mathbb{C}\right\} .
$$

Further for $t \in \mathbb{R}$ with $|t|<\pi / 2$ we set

$$
a_{t}=\left(\begin{array}{ccc}
\cos t & 0 & -i \sin t \\
0 & 1 & 0 \\
-i \sin t & 0 & \cos t
\end{array}\right) \in \exp (i \Omega)
$$

The statement of the lemma translates into the assertion

$$
G n_{i \sin t} \cdot \mathbf{x}_{0}=G a_{t} \cdot \mathbf{x}_{0} .
$$

Clearly, it suffices to prove that

$$
a_{t} \cdot \mathbf{x}_{0}=(\cos t, 0,-i \sin t)^{T} \in G n_{i \sin t} \cdot \mathbf{x}_{0} .
$$

Now let $k \in K$ and $b \in A$ be elements which we write as

$$
k=\left(\begin{array}{ccc}
1 & 0 & 0 \\
0 & \cos \theta & \sin \theta \\
0 & -\sin \theta & \cos \theta
\end{array}\right) \text { and } b=\left(\begin{array}{ccc}
\cosh r & 0 & \sinh r \\
0 & 1 & 0 \\
\sinh r & 0 & \cosh r
\end{array}\right)
$$

for real numbers $r, \theta$. For $y \in \mathbb{R}$, a simple computation yields that

$$
k b n_{i y} \cdot \mathbf{x}_{0}=\left(\begin{array}{c}
\cosh r\left(1-\frac{1}{2} y^{2}\right)-\frac{1}{2} y^{2} \sinh r \\
i y \cos \theta+\sin \theta\left(\sinh r\left(1-\frac{1}{2} y^{2}\right)-\frac{1}{2} y^{2} \cosh r\right) \\
-i y \sin \theta+\cos \theta\left(\sinh r\left(1-\frac{1}{2} y^{2}\right)-\frac{1}{2} y^{2} \cosh r\right)
\end{array}\right) .
$$

Now we make the choice of $\theta=\pi / 2$ which gives us that

$$
k b n_{i y} \cdot \mathbf{x}_{0}=\left(\begin{array}{c}
\cosh r\left(1-\frac{1}{2} y^{2}\right)-\frac{1}{2} y^{2} \sinh r \\
\sinh r\left(1-\frac{1}{2} y^{2}\right)-\frac{1}{2} y^{2} \cosh r \\
-i y
\end{array}\right) .
$$

As $y=\sin t$ we only have to verify that we can choose $r$ such that $\sinh r\left(1-\frac{1}{2} y^{2}\right)-\frac{1}{2} y^{2} \cosh r=0$. But this is equivalent to

$$
\tanh r=\frac{\frac{1}{2} y^{2}}{1-\frac{1}{2} y^{2}} .
$$


In view of $-1<y=\sin t<1$, the right-hand side is smaller than one and we can solve for $r$.

Let us define a domain in $\mathfrak{n}$

$$
\Lambda=\left\{\left(\begin{array}{ll}
0 & x \\
0 & 0
\end{array}\right) \in \mathfrak{n}|x \in \mathbb{R},| x \mid<1\right\} .
$$

A remarkable consequence of the preceding lemma is the following result which we will establish in full generality later on.

Theorem 3.4. For $G=\operatorname{Sl}(2, \mathbb{R})$ one has

$$
\Xi=G \exp (i \Lambda) \cdot x_{0} \text {. }
$$

Remark 3.5. (a) (Relation to $K N K$ ) As observed by Kostant, for any semisimple Lie group $G$ one has $G=K N K$. As one referee pointed out, a more careful study of the $K N K$-decomposition of $G$ was undertaken by H. Lee Michelson in [Mi]. In particular, Proposition 3.1 in [Mi] applied to $G=\operatorname{Sl}(2, \mathbb{R})$ states that

$$
K\left(\begin{array}{cc}
e^{t} & 0 \\
0 & e^{-t}
\end{array}\right) K=K\left(\begin{array}{cc}
1 & 2 \sinh t \\
0 & 1
\end{array}\right) K
$$

for all $t \in \mathbb{R}$. It is tempting to believe that Lemma 3.3 would follow from some sort of analytic continuation of (3.11). However, we observe a subtle difference in this matter: the location of the even prime. This is surprising and we thank this referee of having raised the question.

(b) It is not a priori clear that $G \exp (i \Lambda) \cdot x_{0}$ is open in $X_{\mathbb{C}}$. This is because of the fact that the natural map

$$
G \times \mathfrak{n} \rightarrow X_{\mathbb{C}}, \quad(g, Y) \mapsto g \exp (i Y) \cdot x_{0}
$$

has singular differential at $(g, 0), g \in G$.

(c) Lemma 3.3 allows us to give a characterization of $\Xi$ as a fiber bundle related to the nilcone. Write $\mathcal{N} \subset \mathfrak{g}$ for the cone of nilpotent elements in $\mathfrak{g}$ and note that $\mathcal{N}=\operatorname{Ad}(K) \mathfrak{n}$. Define a subset of $\Lambda$ by

$$
\Lambda^{+}=\left\{\left(\begin{array}{ll}
0 & x \\
0 & 0
\end{array}\right) \mid x \in \mathfrak{n}, 0 \leq x<1\right\} .
$$

and put $\mathcal{N}^{+}=\operatorname{Ad}(K) \Lambda^{+}$. Then it follows from Lemma 3.3 that the mapping

is a homeomorphism.

$$
G \times{ }_{K} \mathcal{N}^{+} \rightarrow \Xi, \quad[g, Y] \mapsto g \exp (i Y) \cdot x_{0}
$$

While it is not possible to enlarge $\Xi$ to a larger domain in hyperbolic directions, i.e. beyond $\Omega$, the situation is quite different for unipotent elements. 
Lemma 3.6. The differential of the mapping

$$
G \times \mathfrak{n} \rightarrow X_{\mathbb{C}}, \quad(g, Y) \mapsto g \exp (i Y) \cdot x_{0}
$$

is invertible at all points $(g, Y) \in G \times \mathfrak{n}$ with $Y \neq 0$.

Proof. By $G$-equivariance of the map, it will be the sufficient to show that the map is submersive at all points $(\mathbf{1}, Y)$ with $Y \neq 0$. This assertion in turn translates into the identity

$$
e^{-i \operatorname{ad} Y} \mathfrak{g}+i \mathfrak{n}+\mathfrak{k}_{\mathbb{C}}=\mathfrak{g}_{\mathbb{C}}
$$

which is satisfied whenever $Y \neq 0$.

For $a<b$ we define an open subset of $\mathfrak{n}$ by

$$
\Lambda_{a, b}=\left\{\left(\begin{array}{ll}
0 & x \\
0 & 0
\end{array}\right) \in \mathfrak{n} \mid x \in \mathbb{R}, a<x<b\right\},
$$

and declare $G$-invariant connected subsets of $X_{\mathbb{C}}$ by

$$
\Xi_{a, b}=G \exp \left(i \Lambda_{a, b}\right) \cdot x_{0} .
$$

Of further interest for us is the limiting object for $a \rightarrow-\infty, b \rightarrow \infty$,

$$
\Xi_{N}=G \exp (i \mathfrak{n}) \cdot x_{0}=G N_{\mathbb{C}} \cdot x_{0} .
$$

Lemma 3.7. For all $a<b$, the sets $\Xi_{a, b}$ are open. In particular $\Xi_{N}$ is open.

Proof. If $0 \notin(a, b)$, then the assertion follows from Lemma 3.6. Thus we may assume that $0 \in(a, b)$. Suppose first that $|a|,|b| \leq 1$. Then by Lemma 3.3 there exists a symmetric, i.e. $W=\mathbb{Z}_{2}$-invariant, interval $\Omega_{a, b} \subset \Omega$ such that $\Xi_{a, b}=G \exp \left(i \Omega_{a, b}\right) \cdot x_{0}$. The latter set is open by Remark 2.2. Finally assume that $b>1$ or $a<-1$. Then

$$
\Xi_{a, b}=\Xi_{\max \{a,-1\}, \min \{1, b\}} \cup G \exp \left(i \Lambda_{a, b} \backslash\{0\}\right) \cdot x_{0}
$$

is the union of two open sets (use Lemma 3.6 for the second term) and we conclude the proof of the lemma.

We exhibit the structure of the domain $\Xi_{N}$. For that it is useful to move to the hyperboloid picture with $G=\mathrm{SO}_{e}(1,2)$. Define the horocycle space of $X$ by

$$
\operatorname{Hor}(X)=\left\{\xi \in \mathbb{R}^{3} \mid Q(\xi)=0, \xi_{0}>0\right\},
$$

and notice that the map

$$
G / N \rightarrow \operatorname{Hor}(X), \quad g N \mapsto g \cdot \xi_{0}
$$

with $\xi_{0}=(1,0,1)^{T}$ is a diffeomorphism. Let us denote by

$$
\mathbf{z} \cdot \mathbf{w}=z_{0} w_{0}-z_{1} w_{1}-z_{2} w_{2}
$$

the complex bilinear form obtained from polarizing $Q$.

Proposition 3.8. For the domain $\Xi_{N}$ the following assertions hold: 
(i) $\Xi_{N}=\left\{\mathbf{z} \in X_{\mathbb{C}} \mid \mathbf{z} \cdot \xi=1\right.$ for some $\left.\xi \in \operatorname{Hor}(X)\right\}$;

(ii) $\Xi_{N}=X_{\mathbb{C}}-\partial_{d} \Xi-\left\{\mathbf{z} \in X_{\mathbb{C}} \mid Q(\mathbf{x})>0, x_{0}<0\right\}$;

(iii) For all $z \in \Xi_{N}$, the $G$-stabilizer

$$
G_{z}=\{g \in G \mid g . z=z\}
$$

is a compact subgroup of $G$.

Proof. (i) We only have to notice that

$$
N_{\mathbb{C}} \cdot x_{0}=\left\{\mathbf{z} \in X_{\mathbb{C}} \mid \mathbf{z} \cdot \xi_{0}=1\right\} .
$$

(ii) We use the characterization of $\Xi_{N}$ from (i). We have to show that

$$
\Xi_{N}=\left\{\mathbf{z}=\mathbf{x}+i \mathbf{y} \in X_{\mathbb{C}} \mid \mathbf{x} \neq 0\right\} .
$$

For elements $z \in X_{\mathbb{C}}$ we will distinguish three cases: $Q(\mathbf{x})>0, Q(\mathbf{x})<0$ and $Q(\mathbf{x})=0$. Before we do our case by case analysis let us mention the fact that elements $\mathbf{z}=\mathbf{x}+i \mathbf{y} \in \mathbb{C}^{3}$ belong to $X_{\mathbb{C}}$ precisely when

$$
Q(\mathbf{x})-Q(\mathbf{y})=1 \quad \text { and } \quad \mathbf{x} \cdot \mathbf{y}=0 .
$$

Case 1: $Q(\mathbf{x})>0$ and $x_{0}<0$. We claim that the $G$-orbit through $\mathbf{z}$ has a representative of the type $\mathbf{z}=\left(x_{0}, i y_{1}, 0\right)^{T}$. In fact, as $Q(\mathbf{x})>0$, the $G$-orbit through $\mathbf{x}$ has a representative $\left(x_{0}, 0,0\right)^{T}$ with $x_{0}<0$. From $\mathbf{x} \cdot \mathbf{y}=0$ we then conclude that $\mathbf{y}=\left(0, y_{1}, y_{2}\right)^{T}$. Further we may alter $\mathbf{y}$ by the stabilizer $K=\mathrm{SO}(2, \mathbb{R})$ of $\mathbf{x}$. Thus we may assume that $\mathbf{y}=\left(0, y_{1}, 0\right)^{T}$. But then $\mathbf{z} \cdot \xi=1$ for $\xi=\left(1 / x_{0}, 0,1 / x_{0}\right)^{T} \in \operatorname{Hor}(X)$. In particular

$$
\left\{z \in X_{\mathbb{C}} \mid Q(\mathbf{x})>0\right\} \subset \Xi_{N} .
$$

Case 2: $Q(\mathbf{x})<0$. We claim that the $G$-orbit through $\mathbf{z}$ has a representative of the type $\mathbf{z}=\left(0, i y_{1}, x_{2}\right)^{T}$. Indeed, as $Q(\mathbf{x})<0$, we may assume that $\mathbf{x}=\left(0,0, x_{2}\right)^{T}$ with $x_{2}>0$. Orthogonality $\mathbf{x} \cdot \mathbf{y}=0$ then implies that $\mathbf{y}=\left(y_{0}, y_{1}, 0\right)^{T}$. Notice that

$$
Q(\mathbf{y})=y_{0}^{2}-y_{1}^{2}=-1-x_{2}^{2}<0 .
$$

It is allowed to change $\mathbf{y}$ by displacements of $H=\mathrm{SO}(1,1)$, the stabilizer of $\mathbf{x}$. As $H$ acts transitively on all connected components of the level sets of $y_{0}^{2}-y_{1}^{2}$, it is no loss of generality to assume that $\mathbf{y}=\left(0, y_{1}, 0\right)^{T}$. But then $\mathbf{z} \cdot \xi=1$ for $\xi=\left(1 / x_{2}, 0,-1 / x_{2}\right)^{T} \in \operatorname{Hor}(X)$ and we conclude that

$$
\left\{z \in X_{\mathbb{C}} \mid Q(\mathbf{x})<0\right\} \subset \Xi_{N} .
$$

Case 3: $Q(\mathbf{x})=0$ and $\mathbf{x} \neq 0$. We assert that the $G$-orbit through $\mathbf{z}$ has a representative of the type $\mathbf{z}=\left(1+i y_{0}, 1+i y_{0}, \pm 1\right)^{T}$. Namely, as $Q(\mathbf{x})=0$ and $\mathbf{x} \neq 0$, the $G$-orbit through $\mathbf{x}$ contains the element $\mathbf{x}=(1,1,0)^{T}$. Then $\mathbf{x} \cdot \mathbf{y}=0$ and $Q(\mathbf{y})=-1$ force $y=\left(y_{0}, y_{0}, \pm 1\right)$. Hence we can choose $\mathbf{z}$ of the asserted form. But then $\xi=\left(\frac{y_{0}^{2}+1}{2}, \frac{y_{0}^{2}-1}{2}, \mp y_{0}\right)^{T} \in \operatorname{Hor}(X)$ with $\mathbf{z} \cdot \xi=1$. 
Finally, we observe that elements of the type $\mathbf{z}=i \mathbf{y}$ cannot belong to $\Xi_{N}$ by (i).

(iii) Notice that $g \cdot \mathbf{z}=\mathbf{z}$ means that $g \cdot \mathbf{x}=\mathbf{x}$ and $g \cdot \mathbf{y}=\mathbf{y}$. We analyze the three cases in (ii). If $Q(\mathbf{x})>0$, then $G . \mathbf{x} \simeq X$ and $G_{\mathbf{x}}$ is compact. If $Q(\mathbf{x})<0$, then we may assume that $\mathbf{z}=\left(0, i y_{1}, x_{2}\right)^{T}$. Hence $g \cdot \mathbf{x}=\mathbf{x}$ forces $g \in H$ and then $g \cdot \mathbf{y}=\mathbf{y}$ yields $g=\mathbf{1}$. Finally if $Q(\mathbf{x})=0$ and $\mathbf{x} \neq 0$, then our choice of $\mathbf{z}$ can be $\mathbf{z}=\left(1+i y_{0}, 1+i y_{0}, \pm 1\right)^{T}$. Then $g \cdot \mathbf{x}=\mathbf{x}$ implies that $g$ is unipotent while $g \cdot \mathbf{y}=\mathbf{y}$ forces $g$ to be hyperbolic. Hence $g=\mathbf{1}$ in this case also.

The statement in Proposition 3.8 (iii) suggest that the $G$-action on $\Xi_{a, b}$ should be proper. However, this is not always the case as our next result shows.

Proposition 3.9. Suppose that $(a, b) \cap(-1,1) \neq \emptyset$. Then the following assertions hold:

(i) If $\max \{|a|,|b|\} \leq 1$, then the $G$-action on $\Xi_{a, b}$ is proper.

(ii) If $\min \{|a|,|b|\}>1$, then the $G$-action on $\Xi_{a, b}$ is not proper.

Proof. If $|a|,|b| \leq 1$, then $\Xi_{a, b} \subset \Xi$ and as the $G$-action is proper on $\Xi$, the same holds for $\Xi_{a, b}$. We move to (ii). Assume now that $|a|>1$ and $|b|>1$. Then $\Xi_{a, b}$ contains both elements $\mathbf{w}_{+}=n_{i} \cdot \mathbf{x}_{0}$ and $\mathbf{w}_{-}=n_{-i} \cdot \mathbf{x}_{0}$. We note that

$$
\mathbf{w}_{+}:=\left(\begin{array}{c}
1 / 2 \\
i \\
-1 / 2
\end{array}\right)=n_{i} \cdot \mathbf{x}_{0}=\left(\begin{array}{ccc}
1 / 2 & i & 1 / 2 \\
i & 1 & -i \\
-1 / 2 & i & 1 / 2
\end{array}\right) \cdot\left(\begin{array}{l}
1 \\
0 \\
0
\end{array}\right) .
$$

For $n \in \mathbb{N}$ we define elements $\mathbf{z}_{n} \in X_{\mathbb{C}}$ by

$$
\mathbf{z}_{n}=\left(1 / 2,0,-1 / 2+e^{-n}\right)^{T}+i\left(0, \sqrt{3 / 4+\left(e^{-n}-1 / 2\right)^{2}} 0\right)^{T} .
$$

Notice that $\lim _{n \rightarrow \infty} \mathbf{z}_{n}=\mathbf{w}_{+}$. Hence there exists an $n_{0} \in \mathbb{N}$ such that $\mathbf{z}_{n} \in \Xi_{a, b}$ for all $n \geq n_{0}$. Now set

$$
b_{n}=\left(\begin{array}{ccc}
\cosh n & 0 & \sinh n \\
0 & 1 & 0 \\
\sinh n & 0 & \cosh n
\end{array}\right) \in A .
$$

Note that eigenvectors of $b_{n}$ are

$$
\mathbf{f}_{1}=\left(\begin{array}{l}
1 \\
0 \\
1
\end{array}\right), \quad \mathbf{f}_{2}=\left(\begin{array}{l}
0 \\
1 \\
0
\end{array}\right), \quad \mathbf{f}_{3}=\left(\begin{array}{c}
1 \\
0 \\
-1
\end{array}\right),
$$

with eigenvalues $e^{n}, 1$ and $e^{-n}$ respectively. Thus

$$
b_{n} \cdot \mathbf{z}_{n}=b_{n} \cdot \mathbf{x}_{\mathbf{n}}+i \mathbf{y}_{n}
$$




$$
\begin{aligned}
= & e^{n} \cdot \frac{x_{0, n}+x_{2, n}}{2} \cdot \mathbf{f}_{1}+e^{-n} \cdot \frac{x_{0, n}-x_{2, n}}{2} \cdot \mathbf{f}_{2} \\
& \quad+i \sqrt{3 / 4+\left(e^{-n}-1 / 2\right)^{2}} \cdot \mathbf{f}_{3} \\
= & 1 / 2 \cdot \mathbf{f}_{1}+e^{-n}\left(1-e^{-n} / 2\right) \cdot \mathbf{f}_{2}+i \sqrt{3 / 4+\left(e^{-n}-1 / 2\right)^{2}} \cdot \mathbf{f}_{3},
\end{aligned}
$$

and thus $\lim _{n \rightarrow \infty} b_{n} \cdot \mathbf{z}_{n}=\mathbf{w}_{-} \in \Xi_{a, b}$. Hence $\left(b_{n} \cdot \mathbf{z}_{n}\right)_{n \geq n_{0}}$ stays in a compact subset of $\Xi_{a, b}$ but with $\left(b_{n}\right)_{n \geq n_{0}}$ an unbounded sequence. Thus the action of $G$ on $\Xi_{a, b}$ is not proper.

We conclude this section with a final result for proper $G$-action.

Proposition 3.10. Let $G=\operatorname{Sl}(2, \mathbb{R})$ and let $D \subset X_{\mathbb{C}}$ be a $G$-invariant domain with $X \subset D$. If the action of $G$ on $D$ is proper, then

(i) $\partial_{s} \Xi \cap D=\emptyset$;

(ii) $\partial_{n} \Xi \nsubseteq D$.

In particular, if $\partial_{n} \Xi \cap D=\emptyset$, then $D \subseteq \Xi$.

Proof. Let $X \subset D \subset X_{\mathbb{C}}$ be an open $G$-invariant domain with proper $G$-action. Suppose that $D \cap \partial \Xi \neq \emptyset$ and let $z$ be a point thereof. Then $z \notin \partial_{d} \Xi=G / H$ as $H$ is noncompact and the $G$-action on $D$ is proper. Hence $z \in \partial_{n} \Xi$. It follows from Theorem 3.1 that

$$
\partial_{n} \Xi=G n_{i} \cdot \mathbf{x}_{0} \amalg G n_{-i} \cdot \mathbf{x}_{0} .
$$

Thus $D \cap \Xi_{a, b} \neq \emptyset$ for some $a, b$ with $\max \{|a|,|b|\}>1$ - the assertion now follows from the previous proposition.

Remark 3.11. There exist larger $G$-domains $D \supsetneq \Xi$ with the $G$-action proper. We provide the recipe for their construction in case of $G=\operatorname{Sl}(2, \mathbb{R})$. Recall that $X$ identifies with the upper halfplane and henceforth we view $X$ in the projective space $\mathbb{P}^{1}(\mathbb{C})$. Notice that $G_{\mathbb{C}}$ acts on $\mathbb{P}^{1}(\mathbb{C})$ by fractional linear transformation. Denote by $\bar{X}$ the lower half plane and notice that $\Xi$ is $G$-isomorphic to $X \times \bar{X}$. In this realization $X$ sits in $\Xi=X \times \bar{X}$ via $z \mapsto(z, \bar{z})$. We view $\Xi \in \mathbb{P}^{1}(\mathbb{C}) \times \mathbb{P}^{1}(\mathbb{C})$ and note that

$$
X_{\mathbb{C}}=\left\{(z, w) \in \mathbb{P}^{1}(\mathbb{C}) \times \mathbb{P}^{1}(\mathbb{C}) \mid z \neq w\right\} .
$$

Furthermore

$$
\partial_{s} \Xi=\left\{(x, y) \in \mathbb{P}^{1}(\mathbb{R}) \times \mathbb{P}^{1}(\mathbb{R}) \mid x \neq y\right\}
$$

and

$$
\partial_{n} \Xi=X \times \mathbb{P}^{1}(\mathbb{R}) \amalg \mathbb{P}^{1}(\mathbb{R}) \times \bar{X} .
$$

In particular we see that

$$
D=\left(X \times \mathbb{P}^{1}(\mathbb{C})\right) \cap X_{\mathbb{C}}
$$

provides a $G$-domain in $X_{\mathbb{C}}$ such that

- $\partial_{s} \Xi \cap D=\emptyset$; 
- $\partial_{n} \Xi \not \subset D ;$

- $G$ acts properly on $D$.

With this picture of $\Xi$ one can easily sharpen Proposition 3.9 to: $G$ acts properly on $\Xi_{a, b}$ if and only if $\min \{|a|,|b|\} \leq 1$.

\section{Properness and Maximality of Holomorphic Extension}

The first part of this section is valid for general $G$; the subsection after for $G=\operatorname{Sl}(2, \mathbb{R})$ only.

As we mentioned earlier in Remark 2.3(ii), it was proved in [AG], that $\Omega$ is maximal with respect to proper $G$-action. We will refine this result in Theorem 4.1 below. This new geometric fact translates into a maximality assertion for holomorphic extension of representations.

Theorem 4.1. Let $X \subset D$ be a $G$-domain in $X_{\mathbb{C}}$ with the $G$-action proper. Then the following assertions hold:

(i) $\partial_{s} \Xi \cap D=\emptyset$.

(ii) $\partial_{n} \Xi \not \subset D$. In particular, if $\partial_{n} \Xi \cap D=\emptyset$, then $D \subset \Xi$.

Proof. (i) It was shown in [AG] that $G$-stabilizers on $\partial_{s} \Xi$ are noncompact. Hence the assertion.

(ii) Suppose that $\partial_{n} \Xi \subset D$ and let $z$ be a point of $\partial_{n} \Xi$. As $D$ is open we may assume that $z$ is generic in the sense of [FH, §2]. It follows from [FH, Th. 4.3.5], that there is a subgroup $G_{0} \subset G$ which is locally isomorphic to $\operatorname{Sl}(2, \mathbb{R})$ such that the crown $\Xi_{0}$ associated to $G_{0}$ embeds $G_{0}$-equivariantly into $\Xi$ with $z \in \partial_{n} \Xi_{0}$ in addition. As $\partial_{n} \Xi_{0} \subset \partial_{n} \Xi$ we obtain a contradiction to Proposition 3.10.

We turn to applications in representation theory. For that it is convenient to look at the preimage

of $\Xi$ in $G_{\mathbb{C}}$.

$$
\tilde{\Xi}=G \exp (i \pi / 2 \Omega) K_{\mathbb{C}}
$$

We let $(\pi, \mathcal{H})$ be a unitary irreducible representation of $G$ and write $\mathcal{H}_{K}$ for the associated Harish-Chandra module of $K$-finite vectors. Then, for $v \in \mathcal{H}_{K}$, it was shown in [KrS1] that the orbit map

$$
F_{v}: G \rightarrow \mathcal{H}, \quad g \mapsto \pi(g) v
$$

extends to a $G$-equivariant holomorphic map $\tilde{\Xi} \rightarrow \mathcal{H}$, also denoted by $F_{v}$ in the sequel. We wish to show that $\tilde{\Xi}$ is maximal and want to relate this to the properness of the action of $G$ on $\Xi$. The link is established through the following fact. 
Lemma 4.2. Let $(\pi, \mathcal{H})$ be a unitary representation of a reductive group $G$ which does not contain the trivial representation. Then $G$ acts properly on $\mathcal{H}-\{0\}$.

Proof. Let $C \subset \mathcal{H}-\{0\}$ be a compact subset and $C_{G}=\{g \in G \mid \pi(g) C \cap$ $C \neq \emptyset\}$. Suppose that $C_{G}$ is not compact. Then there exists a sequence $\left(g_{n}\right)_{n \in \mathbb{N}}$ in $C_{G}$ and a sequence $\left(v_{n}\right)_{n \in \mathbb{N}}$ in $C$ such that $\pi\left(g_{n}\right) v_{n} \in C$ and $\lim _{n \rightarrow \infty} g_{n}=\infty$. As $C$ is compact we may assume that $\lim _{n \rightarrow \infty} v_{n}=v$ and $\lim _{n \rightarrow \infty} \pi\left(g_{n}\right) v_{n}=w$ with $v, w \in C$. We claim that

$$
\lim _{n \rightarrow \infty}\left\langle\pi\left(g_{n}\right) v, w\right\rangle \neq 0 \text {. }
$$

In fact $\left\|\pi\left(g_{n}\right) v_{n}-\pi\left(g_{n}\right) v\right\|=\left\|v_{n}-v\right\| \rightarrow 0$ and thus $\pi\left(g_{n}\right) v \rightarrow w$ as well. As $w \in C$, it follows that $w \neq 0$ and our claim is established.

Finally we observe that (4.1) contradicts the Riemann-Lebesgue lemma $[\mathrm{HoM}]$ for representations which asserts that the matrix coefficient vanishes at infinity.

From Lemma 4.2 we deduce the following result.

Theorem 4.3. Let $(\pi, \mathcal{H})$ be an irreducible unitary representation of $G$ which is not trivial. Let $v \in \mathcal{H}_{K}, v \neq 0$, be a $K$-finite vector. Let $\tilde{D}$ be

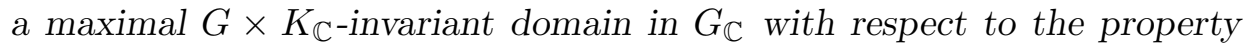
that the orbit map $F_{v}: G \rightarrow \mathcal{H}, g \mapsto \pi(g) v$ extends to a $G$-equivariant holomorphic map $\tilde{\Xi} \rightarrow \mathcal{H}$. Then $G$ acts properly on $\tilde{D} / K_{\mathbb{C}} \subset X_{\mathbb{C}}$.

Proof. We argue by contradiction and assume that $G$ does not act properly on $D=\tilde{D} / K_{\mathbb{C}}$. We obtain sequences $\left(z_{n}^{\prime}\right)_{n \in \mathbb{N}} \subset D$ and $\left(g_{n}\right)_{n \in \mathbb{N}} \subset G$ such that $\lim _{n \rightarrow \infty} z_{n}^{\prime}=z^{\prime} \in D, \lim _{n \rightarrow \infty} g_{n} z_{n}^{\prime}=w^{\prime} \in D$ and $\lim _{n \rightarrow \infty} g_{n}=\infty$. We select preimages $z_{n}, z$ and $w$ of $z_{n}^{\prime}, z^{\prime}$ and $w^{\prime}$ in $\tilde{D}$. We may assume that $\lim _{n \rightarrow \infty} z_{n}=z$ and find a sequence $\left(k_{n}\right)_{n \in \mathbb{N}}$ in $K_{\mathbb{C}}$ such that $\lim _{n \rightarrow \infty} g_{n} z_{n} k_{n}=w$.

Before we continue we claim that

$$
(\forall z \in \tilde{D}) \quad \pi(z) v \neq 0 .
$$

In fact assume $\pi(z) v=0$ for some $z \in \tilde{D}$. Then $\pi(g) \pi(z) v=0$ for all $g \in G$. In particular the map $G \rightarrow \mathcal{H}, g \mapsto \pi(g) v$ is constantly zero. However this map extends to a holomorphic map to a $G$-invariant neighborhood in $G_{\mathbb{C}}$. By the identity theorem for holomorphic functions this map has to be zero as well. We obtain a contradiction to $v \neq 0$ and our claim is established.

Write $V=\operatorname{span}\{\pi(K) v\}$ for the finite-dimensional space spanned by the $K$-translates of $v$. In our next step we claim that

$$
\left(\exists c_{1}, c_{2}>0\right) \quad c_{1}<\left\|\pi\left(k_{n}\right) v\right\|<c_{2} .
$$


In fact from

$$
\lim _{n \rightarrow \infty} \pi\left(g_{n} z_{n} k_{n}\right) v=\pi(w) v \quad \text { and } \quad\left\|\pi\left(g_{n} z_{n} k_{n}\right) v\right\|=\left\|\pi\left(z_{n}\right) \pi\left(k_{n}\right) v\right\|,
$$

we conclude with (4.2) that there are positive constants $c_{1}^{\prime}, c_{2}^{\prime}>0$ such that $c_{1}^{\prime}<\left\|\pi\left(z_{n}\right) \pi\left(k_{n}\right) v\right\|<c_{2}^{\prime}$ for all $n$. We use that $\lim _{n \rightarrow \infty} z_{n}=z \in \tilde{D}$ to obtain $\left.\pi\left(z_{n}\right)\right|_{V}-\left.\pi(z)\right|_{V} \rightarrow 0$ and our claim follows.

We define $C$ to be the closure of the sequences $\left(\pi\left(z_{n} k_{n}\right) v\right)_{n \in \mathbb{N}}$ and $\left(\pi\left(g_{n} z_{n} k_{n}\right) v\right)_{n \in \mathbb{N}}$ in $\mathcal{H}$. With our previous claims (4.2) and (4.3) we obtain that $C \subset \mathcal{H}-\{0\}$ is a compact subset. But $C_{G}=\{g \in G \mid \pi(g) C \cap C \neq \emptyset\}$ contains the unbounded sequence $\left(g_{n}\right)_{n \in \mathbb{N}}$ and hence is not compact - a contradiction to Lemma 4.2.

Remark 4.4. Let $(\pi, \mathcal{H})$ and $v \in \mathcal{H}$ be as in the theorem. Then we might ask whether the stronger statement

$$
\lim _{z \rightarrow \partial \tilde{\Xi}}\|\pi(z) v\|=\infty
$$

holds true. For the special case of $v=v_{K} \in \mathcal{H}_{K}$ a $K$-fixed vector this was established in [KrS2, Th. 2.4].

4.1 Domains of holomorphy for the unitary dual of $G=\operatorname{Sl}(2, \mathbb{R})$. Now let $G=\operatorname{Sl}(2, \mathbb{R})$. With the coordinates of Remark 3.11 we have

$$
\begin{gathered}
X_{\mathbb{C}}=\left(\mathbb{P}^{1}(\mathbb{C}) \times \mathbb{P}^{1}(\mathbb{C}) \backslash \operatorname{diag}\left(\mathbb{P}^{1}(\mathbb{C})\right),\right. \\
\Xi=X \times \bar{X},
\end{gathered}
$$

where $X$ denotes the upper and $\bar{X}$ the lower halfplane. Then there are two interesting $G$-domains in $X_{\mathbb{C}}$ which contain $\Xi$. These are

- $S^{+}=\left(\mathbb{P}^{1}(\mathbb{C}) \times \bar{X}\right) \cap X_{\mathbb{C}}$;

- $S^{-}=\left(X \times \mathbb{P}^{1}(\mathbb{C})\right) \cap X_{\mathbb{C}}$.

Proposition 4.5. The following assertions hold:

(i) $S^{+}=G \exp \left(i \Lambda_{(-1, \infty)}\right) \cdot x_{0}$;

(ii) $S^{-}=G \exp \left(i \Lambda_{(-\infty, 1)}\right) \cdot x_{0}$.

In particular, $S^{ \pm}$are maximal $G$-domains in $X_{\mathbb{C}}$ on which $G$ acts properly.

Proof. The first two assertions come down to a very elementary computation; the last one follows from Proposition 3.10.

REMARK 4.6. As $\mathfrak{g}$ is of Hermitian type, the $\mathfrak{k}_{\mathbb{C}}$-module $\mathfrak{p}_{\mathbb{C}}$ splits into two inequivalent subspaces $\mathfrak{p}_{\mathbb{C}}=\mathfrak{p}^{+} \oplus \mathfrak{p}^{-}$with

$$
\mathfrak{p}^{ \pm}=\mathbb{C} \cdot\left(\begin{array}{cc}
1 & \pm i \\
\pm i & 1
\end{array}\right)
$$

Set $P^{ \pm}=\exp \left(\mathfrak{p}^{ \pm}\right)$. Then the preimages $\tilde{S}^{ \pm}$of $S^{ \pm}$in $G_{\mathbb{C}}$ are given by 
- $\tilde{S}^{+}=G K_{\mathbb{C}} P^{+}$

- $\tilde{S}^{-}=G K_{\mathbb{C}} P^{-}$.

We obtain the following result.

Theorem 4.7. Let $(\pi, \mathcal{H})$ be an irreducible unitary representation of $G=\operatorname{Sl}(2, \mathbb{R})$. Let $v \in \mathcal{H}_{K}$ be a non-zero vector and $f_{v}: G \rightarrow \mathcal{H}, g \mapsto \pi(g) v$ the corresponding orbit map. Then the domains of holomorphy of $f_{v}$ are given by

(i) $G_{\mathbb{C}}$, if $\pi$ is trivial;

(ii) $\tilde{S}^{+}$, if $\pi$ is a non-trivial highest weight representation;

(iii) $\tilde{S}^{-}$, if $\pi$ is a non-trivial lowest weight representation;

(iv) $\tilde{\Xi}$, if $\pi$ is none of the above, i.e. a unitarizable principal series.

Proof. (i) is clear.

(ii) If $\mathcal{H}_{K}$ is a highest weight module, then all its vectors are $K_{\mathbb{C}} P^{+}$finite. Hence $\tilde{S}^{+}=G K_{\mathbb{C}} P^{+}$lies in the domain of holomorphy of $f_{v}$. By the preceding Proposition $\tilde{S}^{+}$is maximal for proper action and the assertion follows from Theorem 4.3.

(iii) Analogous to (ii).

(iv) For $\tilde{\Xi}$ to be contained in the domain of holomorphy we refer to the general result of [KrS2, Th.1.1]. If $\pi$ is $K$-spherical, then $\tilde{\Xi}$ is indeed maximal as it follows from [KrOS, Th. 5.1], and Remark 4.8 below. Finally, the case of non-spherical principal series is similar to the spherical case (the same proof as in [KrOS] applies).

REMARK 4.8 (Correcting literature). In the proof of Theorem 5.1 in [KrOS] there is an inaccuracy which we wish to correct here. Actually we have to address the proof of the key result Theorem 5.4 in [KrOS]: it asserts for $G=\operatorname{Sl}(2, \mathbb{R})$ that a spherical function with imaginary parameter blows up at the boundary of $\Xi$. Now $\partial \Xi=\partial_{s} \Xi \amalg \partial_{n} \Xi$. The arguments given for the blow-up at the semisimple boundary $\partial_{s} \Xi$ are fine; the ones for the blow-up at $\partial_{n} \Xi$ are not correct and should be modified. With the notation of [KrOS] we have for $a_{r}=\left(\begin{array}{cc}r & 0 \\ 0 & 1 / r\end{array}\right) \in A, r>0$, and $-1<t<1$, that

$$
P\left(a_{r}\left(\begin{array}{cc}
1 & i t \\
0 & 1
\end{array}\right) \cdot x_{0}\right)=r^{2}+\frac{1}{r^{2}}-t^{2} r^{2} .
$$

In particular, if $|t|>1$, then there would exist a sequence $r_{n} \rightarrow r_{0}$ such that $P\left(a_{r_{t}}\left(\begin{array}{cc}1 & \text { it } \\ 0 & 1\end{array}\right)\right) \rightarrow-2^{+}$. Now we can use the argument given in the proof of Theorem 5.4 in [KrOS]. 
Secondly, let us mention that Theorem 5.1 and Theorem 5.4 are true for all positive definite spherical functions $\neq \mathbf{1}$, not only for those with imaginary parameters as stated - the argument is literally the same.

REMARK 4.9. Theorem 4.7 says that there are different domains of holomorphy for different series of representations. An analogous result for arbitrary semisimple Lie groups was established after completion of this paper, see $[\mathrm{Kr}]$.

\section{Holomorphic Extension of Spherical Functions}

This section is a short essay on spherical functions on $X$ which highlights their natural holomorphic extension to the crown $\Xi$. Here $G$ is arbitrary semisimple (within our self-imposed restrictions).

As always some notation upfront. For $\alpha \in \Sigma$ write $\mathfrak{g}^{\alpha}$ for the corresponding root space. Choose a positive system $\Sigma^{+}$and define $\mathfrak{n}=$ $\sum_{\alpha \in \Sigma^{+}} \mathfrak{g}^{\alpha}$. Set $N=\exp \mathfrak{n}$. The Iwasawa decomposition $G=N A K$ yields the analytic diffeomorphism

$$
N \times A \stackrel{\simeq}{\longrightarrow} X, \quad(n, a) \rightarrow \text { na. } x_{0} .
$$

In particular, every $x \in X$ can be uniquely written as $x=n(x) a(x) \cdot x_{0}$ with $n(x) \in N$ and $a(x) \in A$ both depending analytically on $x$.

Let $N_{\mathbb{C}}=\exp \mathfrak{n}_{\mathbb{C}}$. If we complexify the Iwasawa decomposition of $X$ we obtain a Zariski open subset $N_{\mathbb{C}} A_{\mathbb{C}} \cdot x_{0} \subsetneq X_{\mathbb{C}}$ which contains the crown, i.e.

$$
\Xi \subset N_{\mathbb{C}} A_{\mathbb{C}} \cdot x_{0}
$$

(see $[\mathrm{KrS1} 1$ for classical groups and $[\mathrm{Hu} 1]+[\mathrm{Hu} 2]$ as well as [Mat] in general). Let us mention that $\Omega \subset \mathfrak{a}$ is a maximal domain for the inclusion (5.2) to hold, i.e. $G \exp (i \pi \tilde{\Omega} / 2) \not \subset N_{\mathbb{C}} A_{\mathbb{C}} \cdot x_{0}$ for any domain $\tilde{\Omega} \subset \mathfrak{a}$ strictly containing $\Omega$ (cf. [B] and [KrS2, Th. 2.4] with proof).

Define the finite 2-group $F=A_{\mathbb{C}} \cap K_{\mathbb{C}}=T \cap K$ and record that the map

$$
N_{\mathbb{C}} \times A_{\mathbb{C}} / F \stackrel{\simeq}{\longrightarrow} N_{\mathbb{C}} A_{\mathbb{C}} \cdot x_{0} \quad(n, a F) \mapsto n a \cdot x_{0}
$$

is biholomorphic. It follows that each element $z \in N_{\mathbb{C}} A_{\mathbb{C}} \cdot x_{0}$ can be uniquely expressed as $z=n_{\mathbb{C}}(z) a_{\mathbb{C}}(z) \cdot x_{0}$ with $n_{\mathbb{C}}(z) \in N_{\mathbb{C}}$ and $a_{\mathbb{C}}(z) \in A_{\mathbb{C}} / F$ both holomorphic in $z$. One obtains an $N$-invariant holomorphic assignment

$$
a_{\mathbb{C}}: \Xi \rightarrow A_{\mathbb{C}} / F
$$

We have already remarked that $\Xi$ is contractible and this yields that $a_{\mathbb{C}}$ lifts to a holomorphic map $\Xi \rightarrow A_{\mathbb{C}}$, as well denoted by $a_{\mathbb{C}}$, such that $a_{\mathbb{C}}\left(x_{0}\right)=e$. Likewise there is a holomorphic logarithm $\log a_{\mathbb{C}}: \Xi \rightarrow \mathfrak{a}_{\mathbb{C}}$ 
extending $\log a: X \rightarrow \mathfrak{a}$. In particular, for all $\lambda \in \mathfrak{a}_{\mathbb{C}}{ }^{*}$ we can define the holomorphic $\lambda$-power of $a_{\mathbb{C}}$ by

$$
a_{\mathbb{C}}(z)^{\lambda}=e^{\lambda\left(\log a_{\mathbb{C}}(z)\right)} \quad(z \in \Xi) .
$$

We would like to mention the complex convexity theorem ([GiK2], $[\mathrm{KrO}])$ which states that

$$
\operatorname{Im} \log a_{\mathbb{C}}\left(G \exp (i Y) \cdot x_{0}\right)=\operatorname{co}(W . Y) \quad(Y \in \pi \Omega / 2)
$$

with $\operatorname{co}(\cdot)$ denoting the convex hull of $(\cdot)$. As a consequence we obtain a refinement of the inclusion (5.2):

$$
\Xi \subset N_{\mathbb{C}} A T_{\Omega} \cdot x_{0} .
$$

For $\alpha \in \Sigma$ let us define $m_{\alpha}=\operatorname{dim} \mathfrak{g}^{\alpha}$ and note that the multiplicity assignment $\alpha \mapsto m_{\alpha}$ is $W$-invariant. As usual we set $\rho=\frac{1}{2} \sum_{\alpha \in \Sigma^{+}} m_{\alpha} \alpha$. Motivated by our previous discussion we define the spherical function with parameter $\lambda \in \mathfrak{a}_{\mathbb{C}}{ }^{*}$ ab initio as a holomorphic function on $\Xi$ :

$$
\phi_{\lambda}(z)=\int_{K} a_{\mathbb{C}}(k z)^{\rho+i \lambda} d k \quad(z \in \Xi) .
$$

Of later relevance for us will be the doubling formula for spherical functions ([KrS1, Th.4.2]). For the convenience of the reader we briefly recall the short argument. We translate the inclusion (5.2) into representation theory: Using the compact realization of a spherical minimal principal series module $\left(\pi_{\lambda}, \mathcal{H}_{\lambda}\right)$ one shows that the orbit map of a spherical vector $v_{\lambda} \in \mathcal{H}_{\lambda}$

$$
F: X \rightarrow \mathcal{H}_{\lambda}, \quad x \rightarrow \pi_{\lambda}(x) v_{\lambda}
$$

extends to a holomorphic map

$$
F: \Xi \rightarrow \mathcal{H}_{\lambda} \quad z \rightarrow \pi_{\lambda}(z) v_{\lambda}
$$

see [KrS1, Prop. 4.1]. This allows us to express the spherical function $\phi_{\lambda}$ as a holomorphic matrix coefficient $\phi_{\lambda}(z)=\left\langle v_{\lambda}, \pi_{\lambda}(z) v_{\lambda}\right\rangle$ for $z \in \Xi$ (where we have adopted the physicists' convention that sesquilinear pairings are linear on the right-hand side, and anti-linear on the left-hand side).

Now let $z \in A T_{\Omega}$ such that $z^{2} \in A T_{\Omega}$ and observe that

$$
\phi_{\lambda}\left(z^{2}\right)=\left\langle\pi_{\lambda}^{*}\left(\overline{z^{-1}}\right) v_{\lambda}, \pi_{\lambda}(z) v_{\lambda}\right\rangle
$$

with $\pi_{\lambda}^{*}$ the conjugate contragredient representation. It follows that $\left.\phi_{\lambda}\right|_{A T_{\Omega}}$ extends to a holomorphic function on $A T_{\Omega}^{2}$. In particular we see from this formula in the case of the unitary spherical minimal principal series $\lambda \in \mathfrak{a}^{*}$ that the function $\phi_{\lambda}$ is positive on $T_{\Omega}^{2}$, and that for all $x=g t . x_{0} \in \Xi$ (recall the notation $\left.v_{\lambda}^{x}:=\pi_{\lambda}(x) v_{\lambda}\right)$ :

$$
\begin{aligned}
\left\langle v_{\lambda}^{x}, v_{\lambda}^{x}\right\rangle & =\phi_{\lambda}\left(t^{2}\right) \\
& =\int_{K}\left|a_{\mathbb{C}}(k t)^{2(\rho+i \lambda)}\right| d k .
\end{aligned}
$$




\section{Sharp Uniform Lower Bound for Holomorphically Extended Orbit Maps of Spherical Representations}

Given a non-trivial unitary spherical representation $(\pi, \mathcal{H})$ of $G$ with normalized $K$-spherical vector $v_{K}$ we wish to control the norm of the holomorphically extended orbit map

$$
F_{\pi}: \Xi \rightarrow \mathbb{C}, \quad z \mapsto \pi(z) v_{K}
$$

in two aspects:

- For $z \in \Xi$ sufficiently close to $\partial_{d} \Xi$ we are aiming to give optimal lower bounds for $\left\|F_{\pi}(z)\right\|$ uniform in the representation parameter $\lambda(\pi) \in \mathfrak{a}_{\mathbb{C}}{ }^{*}$

- For fixed $\pi$ we are looking for optimal upper bounds of $\left\|F_{\pi}(z)\right\|$ for $z$ approaching the distinguished boundary.

In view of the fundamental identity (5.9) we can translate the problems above into growth behavior of analytically continued spherical functions. In this section and the next we will address these two aspects. We begin with the uniform lower bounds.

Fix a distinguished boundary point $t=z_{j}=\exp \left(i \pi \omega_{j} / 2 k_{j}\right) \cdot x_{0}$ of the crown domain. For $0<\epsilon<1$ set

$$
t_{\epsilon}=\exp \left(i(1-\epsilon) \pi \omega_{j} / 2 k_{j}\right) \cdot x_{0} .
$$

The objective of this section is to provide sharp lower estimates for $\phi_{\lambda}\left(t_{\epsilon}^{2}\right)$ which are uniform in $\epsilon$ and $\lambda \in \mathfrak{a}^{*}$. Our approach is based on the doubling identity (5.9) which implies that

$$
\phi_{\lambda}\left(t_{\epsilon}^{2}\right) \geq \int_{U}\left|a_{\mathbb{C}}\left(k t_{\epsilon}\right)^{2(\rho+i \lambda)}\right| d k
$$

where $U$ is any neighborhood of $e \in K$. It turns out that the desired estimate will depend on the nature of the distinguished boundary point $t=z_{j}$, in particular whether $z_{j}$ is minuscule or not. We will treat the minuscule case first and later reduce the general case to the minuscule situation.

If $t=z_{j}$ is minuscule, then $G=Z\left(t^{4}\right)$ by Lemma 2.9, i.e. $t^{4}$ is central. The following lemma, especially seen in the context of (5.4), is quite remarkable.

Lemma 6.1. Let $t=z_{j}$ be a minuscule boundary point and $U$ a connected and simply connected compact neighborhood of $e \in K$ such that $U t \subset N_{\mathbb{C}} A_{\mathbb{C}} \cdot x_{0}$. Then for all $k \in U$ the middle projection $a_{\mathbb{C}}(k t) \in A_{\mathbb{C}}$ is well defined and we have $a_{\mathbb{C}}(k t)=r(k t) t$ with $r(k t) \in A$ continuously depending on $k$. 
Proof. The assertion of the lemma is local and thus it is no loss of generality to assume that $G \subset G_{\mathbb{C}}$ with $G_{\mathbb{C}}$ simply connected. In particular the Cartan involution $\theta: G \rightarrow G$ extends to a holomorphic involution on $G_{\mathbb{C}}$, again denoted by $\theta$. We notice that $G_{\mathbb{C}}{ }^{\theta}=K_{\mathbb{C}}$. Likewise $G_{\mathbb{C}}$ admits a complex conjugation $g \mapsto \bar{g}$ with respect to $G$

Fix $k \in K$. Then $k t=n a k^{\prime}$ for some $n \in N_{\mathbb{C}}, a \in A_{\mathbb{C}}$ and $k^{\prime} \in K_{\mathbb{C}}$. Define $x:=k t \theta(k t)^{-1}$ and note that

$$
x=k t^{2} k^{-1}=n a^{2} \theta(n)^{-1} .
$$

On the other hand, as $t^{4}$ is central,

$$
t^{-4} x=k t^{-2} k^{-1}=\overline{k t^{2} k^{-1}}=\bar{x}=\overline{n a}^{2} \theta(\bar{n})^{-1} .
$$

Combining the information of (6.2) and (6.3) yields

$$
t^{4} \overline{n a}^{2} \theta(\bar{n})^{-1}=x=n a^{2} \theta(n)^{-1} .
$$

Once more we use the fact $t^{4}$ is central and obtain

$$
t^{4} \bar{a}^{2}=\underbrace{\left(\bar{n}^{-1} n\right)}_{\in N_{\mathbb{C}}} a^{2} \underbrace{\theta\left(n^{-1} \bar{n}\right)}_{\in \theta\left(N_{\mathbb{C}}\right)} .
$$

Bruhat implies that $\bar{n}=n$. Consequently $t^{4}=a^{2} \bar{a}^{-2}$, and this forces $a=r(t k) t$ for some $r(t k) \in A$.

Choose $0<\epsilon_{0}<1$ small enough such that $U t_{\epsilon} \subset N_{\mathbb{C}} A_{\mathbb{C}} \cdot x_{0}$ for all $\epsilon \in\left(0, \epsilon_{0}\right)$. In particular $a_{\mathbb{C}}\left(k t_{\epsilon}\right)$ is well defined for all $k \in U$ and $\epsilon \in\left(0, \epsilon_{0}\right)$. As $a_{\mathbb{C}}(k t) \in A t$ for all $k \in U$ by the lemma, linear Taylor approximation yields that there are balls $B_{r}, B_{r^{\prime}}$ in $\mathfrak{a}$ centered at 0 with radii $r, r^{\prime}>0$ such that

$$
a_{\mathbb{C}}\left(k t_{\epsilon}\right) \in t \exp \left(B_{r}\right) \exp \left(i \epsilon B_{r^{\prime}}\right),
$$

for all $k \in U$ and $\epsilon \in\left(0, \epsilon_{0}\right)$. Thus it follows that there exists a constant $c>0$ such that for all $\lambda \in \mathfrak{a}^{*}, k \in U$ and $\epsilon \in\left(0, \epsilon_{0}\right)$ the estimate

holds for some $r^{\prime \prime} \geq r^{\prime}$.

$$
\left|a_{\mathbb{C}}\left(k t_{\epsilon}\right)^{2(\rho+i \lambda)}\right| \geq c e^{\lambda\left(\pi \omega_{j}\right)-r^{\prime \prime} \epsilon|\lambda|}
$$

Proposition 6.2. Let $t=z_{j}=\exp \left(i \pi \omega_{j} / 2\right) \cdot x_{0}$ be a minuscule boundary boundary point of $T_{\Omega}$. Then there exist constants $\epsilon_{0} \in(0,1)$ and $R>0$, $C>0$ such that

$$
\phi_{\lambda}\left(t_{\epsilon}^{2}\right) \geq C \max _{w \in W} e^{\pi \lambda\left(w \omega_{j}\right)(1-R \epsilon)}
$$

for all $\lambda \in \mathfrak{a}^{*}$ and $\epsilon \in\left(0, \epsilon_{0}\right)$.

Proof. According to Harish-Chandra one has $\phi_{\lambda}=\phi_{w \lambda}$ for all $\lambda$. Thus it is no loss of generality to assume that $\lambda\left(\omega_{j}\right)=\max _{w \in W} \lambda\left(w \omega_{j}\right)$. We notice that $\|\lambda\|:=\max _{w \in W} \lambda\left(w \omega_{j}\right)$ defines a norm on $\mathfrak{a}^{*}$. Hence, by the equivalence of norms on Euclidean spaces, there exist a constant $d>0$ such 
that $|\cdot| \leq d\|\cdot\|$. Now the the assertion follows from (6.5) and the basic lower estimate (6.1).

Let us now turn to the general case where $t=z_{j}=\exp \left(i \pi \omega_{j} / 2 k_{j}\right) \cdot x_{0}$ is an arbitrary extremal boundary point of $T_{\Omega}$. We recall the groups $G_{j}=$ $Z\left(t^{4}\right)$ with Lie algebra $\mathfrak{g}_{j}$. The main result of this section is

Theorem 6.3. Let $t=\exp \left(i \pi \omega_{j} / 2 k_{j}\right)$ be an extremal boundary point of $T_{\Omega}$. Then there exist constants $\epsilon_{0} \in(0,1)$ and $R>0, C>0$ such that

$$
\phi_{\lambda}\left(t_{\epsilon}^{2}\right) \geq C \epsilon^{\left(\operatorname{dim} \mathfrak{g}-\operatorname{dim} \mathfrak{g}_{j}\right) / 4} \max _{w \in W} e^{\pi \lambda\left(w \omega_{j}\right)(1-R \epsilon)}
$$

for all $\lambda \in \mathfrak{a}$, and for all $\epsilon \in\left(0, \epsilon_{0}\right)$.

Proof. First, for $\omega_{j}$ minuscule one has $\mathfrak{g}_{j}=\mathfrak{g}$ and the assertion follows from Proposition 6.2 above. The general case will be reduced to this situation.

We begin with some remarks on the reductive Lie algebra $\mathfrak{g}_{j}$. Recall that $\mathfrak{g}_{j}$ is $\theta$-stable and hence $\mathfrak{g}_{j}=\mathfrak{k}_{j} \oplus \mathfrak{p}_{j}$ with $\mathfrak{k}_{j}=\mathfrak{k} \cap \mathfrak{g}_{j}$ and $\mathfrak{p}_{j}=$ $\mathfrak{p} \cap \mathfrak{g}_{j}$. By definition $\mathfrak{a} \subset \mathfrak{g}_{j}$ and hence $\mathfrak{a}$ is maximal abelian in $\mathfrak{p}_{j}$. Let $\Sigma_{j}=\Sigma\left(\mathfrak{g}_{j}, \mathfrak{a}\right)$ be the corresponding reduced root system and $\Omega_{j} \subset \mathfrak{a}$ the associated polyhedron. A quick look at our classification of the $\mathfrak{g}_{j}$ 's shows that $\mathfrak{g}_{j}$ is simple modulo a compact ideal. Hence $\Sigma_{j}$ is irreducible and $\omega_{j} / k_{j}$ becomes a minuscule boundary point of $\Omega_{j}$.

Write $\mathfrak{k}_{j}^{\perp}$ for the orthogonal complement to $\mathfrak{k}_{j}$ in $\mathfrak{k}$ with respect to the Cartan-Killing form of $\mathfrak{g}$. Let $V_{j}, V_{j}^{\prime}$ be small balls around 0 in $\mathfrak{k}_{j}, \mathfrak{k}_{j}^{\perp}$ such that the map

$$
V_{j} \times V_{j}^{\prime} \rightarrow K, \quad\left(v, v^{\prime}\right) \mapsto \exp (v) \exp \left(v^{\prime}\right)
$$

is a diffeomorphism. Set $U_{j}=\exp \left(V_{j}\right), U_{j}^{\prime}=\exp \left(V_{j}^{\prime}\right)$ and define $U=U_{j} U_{j}^{\prime}$. Then $U$ is a connected and simply connected neighborhood of $e$ in $K$. We assume that $U t \subset N_{\mathbb{C}} A_{\mathbb{C}} \cdot x_{0}$ and choose $\epsilon_{0}>0$ such that $U t_{\epsilon} \subset N_{\mathbb{C}} A_{\mathbb{C}} \cdot x_{0}$ for $\epsilon \in\left(0, \epsilon_{0}\right)$ holds in addition.

Our previous discussion combined with Lemma 6.1 implies that

$$
a_{\mathbb{C}}(k t) \in r(k t) t \quad \forall k \in U_{j}^{\prime}
$$

and $r(k t) \in A$ depending continuously on $k$. Next consider the map $\psi$ : $U \rightarrow A_{\mathbb{C}}, k \mapsto a_{\mathbb{C}}(k t)$. We claim that

$$
d \psi(e)=0 .
$$

In fact, this is well known, and follows from $\operatorname{pr}_{\mathfrak{a}_{\mathbb{C}}}\left(\operatorname{Ad}\left(t^{-1}\right) \mathfrak{k}\right)=\{0\}$ with $\operatorname{pr}_{\mathfrak{a}_{\mathbb{C}}}: \mathfrak{g}_{\mathbb{C}} \rightarrow \mathfrak{a}_{\mathbb{C}}$ the linear projection along $\mathfrak{n}_{\mathbb{C}} \oplus \mathfrak{k}_{\mathbb{C}}$

Using the information of (6.8) and (6.9), linear Taylor approximation yields constants $r, r^{\prime}>0$ such that

$$
a_{\mathbb{C}}\left(\exp (v) \exp \left(v^{\prime}\right) t_{\epsilon}\right) \in t \exp \left(B_{r}\right) \exp \left(i\left(\epsilon+\left\|v^{\prime}\right\|^{2}\right) B_{r^{\prime}}\right)
$$


for all $\left(v, v^{\prime}\right) \in V_{j} \times V_{j}^{\prime}$ and $\epsilon \in\left(0, \epsilon_{0}\right)$. It follows from equation (6.1) that there exists a constant $c>0$ such that

$$
\phi_{\lambda}\left(t_{\epsilon}^{2}\right) \geq c \int_{V_{j}} \int_{V_{j}^{\prime}}\left|a_{\mathbb{C}}\left(\exp (v) \exp \left(v^{\prime}\right) t_{\epsilon}\right)^{2(\rho+i \lambda)}\right| d v d v^{\prime}
$$

for all $\lambda \in \mathfrak{a}^{*}$ and $\epsilon \in\left(0, \epsilon_{0}\right)$. Thus if we choose $V_{j}^{\prime}$ to be ball of radius $\sqrt{\epsilon}$, then (6.10) and (6.11) yield constants $r^{\prime \prime}, c^{\prime}>0$ such that

$$
\phi_{\lambda}\left(t_{\epsilon}^{2}\right) \geq c^{\prime} \epsilon^{\left(\operatorname{dim} \mathfrak{k}_{j}^{\perp}\right) / 2} e^{\lambda\left(\pi \omega_{j} / k_{j}\right)-r^{\prime \prime} \epsilon|\lambda|}
$$

for all $\lambda \in \mathfrak{a}^{*}$ and $\epsilon \in\left(0, \epsilon_{0}\right)$ (note that the $\epsilon$-dependence of $V_{j}^{\prime}$ is incorporated in the factor $\left.\epsilon^{\left(\operatorname{dim} \mathfrak{k}_{j}^{\perp}\right) / 2}\right)$. We observe that $\operatorname{dim} \mathfrak{g}-\operatorname{dim} \mathfrak{g}_{j}=2 \operatorname{dim} \mathfrak{k}_{j}^{\perp}$ and finish the proof with the same argument for Proposition 6.2 as before.

REMARK 6.4. We consider the lower estimate in Theorem 6.3 is optimal. This is for the following reason: the crucial point in the above argumentation was the fact that $d \psi(e)=0$, to be very precise it was the fact $\left.d(\operatorname{Im} \log \psi)(e)\right|_{\mathfrak{k}_{j}^{\perp}}=0$ which entered. This is actually the best one can hope for as the second derivative $d^{2}(\operatorname{Im} \log \psi)(e)$ is already non-degenerate on $\mathfrak{k}_{j}^{\perp} \times \mathfrak{k}_{j}^{\perp}$. In fact, fix $\lambda \in \mathfrak{a}^{*}$ regular, and set $F_{\lambda}=\lambda \circ \operatorname{Im} \log \psi$. Then [DuKV, pp. 343-346] implies

$$
d^{2} F_{\lambda}(e)(Z, W)=-\frac{1}{2} \sum_{\alpha \in \Sigma^{+}}\langle\alpha, \lambda\rangle \operatorname{Im}\left(1-t^{-2 \alpha}\right)\left\langle Z_{\alpha}, W_{\alpha}\right\rangle \quad(Z, W \in \mathfrak{k})
$$

where $Z_{\alpha}$, resp. $W_{\alpha}$ is the orthogonal projection of $Z$, resp. $W$, onto $\mathfrak{k} \cap\left(\mathfrak{g}^{\alpha}+\mathfrak{g}^{-\alpha}\right)$. In particular if $\alpha \in \Sigma \backslash \Sigma_{j}$, then $\operatorname{Im}\left(1-t^{-2 \alpha}\right) \neq 0$. It follows that $d^{2} F_{\lambda}(e)$ and hence $d^{2}(\operatorname{Im} \log \psi)(e)$ is non-degenerate on $\mathfrak{k}_{j}^{\perp} \times \mathfrak{k}_{j}^{\perp}$.

\section{Sharp Upper Bound for Holomorphically Extended Orbit Maps of Spherical Representations}

In this section we consider the problem to give an upper estimate for the square norm of the holomorphic extension of the orbit map $\Xi \ni x \rightarrow v^{x} \in \mathcal{H}$. Recall from (5.9) that

$$
\left(v_{i \lambda}^{x}, v_{i \lambda}^{x}\right)=\phi_{i \lambda}^{X}\left(t^{2} \cdot x_{0}\right)
$$

if $x=$ gt. $x_{0} \in \Xi$ with $g \in G$ and $t \in T_{\Omega}$. Here $\phi_{\mu}^{X}$ denotes the elementary spherical function on $X$ with spectral parameter $\mu \in \mathfrak{a}_{\mathbb{C}}{ }^{*}$. Therefore we concentrate on the question of estimating the singular behavior of the holomorphic extension of the restriction of the elementary spherical function $\left.\phi_{\mu}^{X}\right|_{A}$ to $A_{\mathbb{C}} \supset A$ when we approach $t^{2} \cdot x_{0} \in T \cdot x_{0} \subset A_{\mathbb{C}} \cdot x_{0}$ where $t=t(\eta)=\exp (i \pi \eta / 2)$ with $\eta=\omega_{j} / k_{j} \in \mathfrak{a}_{\mathbb{C}}$ an extremal boundary point of $\Omega$ in $\Omega \cap C$. Thus we are interested in the singular expansion 
in $\epsilon$ of the pull back of (the holomorphic continuation of) spherical functions $\phi_{\mu}$ via the embedding $\mathbb{D}^{\times} \ni \epsilon \rightarrow A_{\mathbb{C}} \cdot x_{0}$ of a small punctured disk $\mathbb{D}_{r}^{\times}=\{\epsilon \in \mathbb{C}|0<| \epsilon \mid<r\}$ given by $\epsilon \rightarrow t_{\epsilon}^{2} \cdot x_{0}$. For $\mu$ fixed, the restriction of $\phi_{\mu}^{X}$ has a convergent logarithmic singular expansion at $\epsilon=0$. This means that there exists a finite set $S \subset \mathbb{C} \times \mathbb{Z}_{\geq 0}$ such that if $(s, l),\left(s^{\prime}, l^{\prime}\right) \in S$ then $s-s^{\prime} \notin \mathbb{Z} \backslash\{0\}$ and such that we have a unique decomposition (for $\epsilon$ varying in any sector $S_{r, \theta_{1}, \theta_{2}}=\left\{\epsilon \in \mathbb{D}_{r}^{\times} \mid \theta_{1}<\arg (\epsilon)<\theta_{2}\right\}$ of $\left.\mathbb{D}_{r}^{\times}\right)$of the form

$$
\phi_{\mu}^{X}\left(t_{\epsilon}^{2} \cdot x_{0}\right)=\sum_{(s, l) \in S} \epsilon^{s} \log ^{l} \epsilon f_{s, l}(\epsilon)
$$

where each $f_{s, l}$ is holomorphic on $\mathbb{D}_{r}$ and such that if $(s, l) \in S$ then there exist $l^{\prime} \in \mathbb{Z}_{\geq 0}$ such that $f_{s, l^{\prime}}(0) \neq 0$.

The projection of the set $S$ on the first factor $\mathbb{C}$ is called the set of exponents of the pull back of $\phi_{\mu}^{X}$ to $\mathbb{D}_{r}^{\times}$. In our case this set will always belong to $\mathbb{R}$. The minimum of this set is denoted by $s_{\eta, \mu}^{X}$ and is called the leading exponent of the singular expansion of the pull back of $\phi_{\mu}^{X}$ to $\mathbb{D}_{r}^{\times}$. We call the largest $l \in \mathbb{Z}_{\geq 0}$ such that there exists a pair $(s, l) \in S$ with $s=s_{\eta, \mu}^{X}$ and such that $f_{(s, l)}(0) \neq 0$ the logarithmic degeneracy of the leading exponent.

So our problem boils down to the determination of the leading exponent $s_{\eta, \mu}^{X}$ at $\epsilon=0$ of the pull back of $\phi_{\mu}^{X}$ on the distinguished embedded punctured disk given above, and its logarithmic degeneracy. In the Appendix 13 we define an appropriate notion of the exponent of a regular holonomic system of differential equations and using the basic properties of these exponents we compute the exponents of $\phi_{\mu}$ at the extremal boundary points $\eta$ of $\Omega \cap C$ for $\phi_{\mu}$ a solution of a more general system of differential equations, namely the system of hypergeometric equations associated with the root system $\Sigma$. This system of equations is a parameter deformation of the system of equations for the restriction for the elementary spherical functions $\phi_{\mu}^{X}$ to $A_{\mathbb{C}} \cdot x_{0}$. This deformation is an essential ingredient for the computations of the exponents. These results imply the following:

Theorem 7.1. We use the notation as introduced above. Consider the functions $s_{\eta}(m)$ and $d_{\eta}(m)$ of the multiplicity parameters $m=\left(m_{\alpha}\right)$ as listed in the table in Theorem 7.9. Suppose that the Riemannian symmetric space $X$ has root system $\Sigma$ and root multiplicity parameters $m^{X}=\left(m_{\alpha}^{X}\right)$, then we put $s_{\eta}^{X}:=s_{\eta}\left(m^{X}\right)$ and $d_{\eta}^{X}=d_{\eta}\left(m^{X}\right)$.

For all $\mu \in \mathfrak{a}_{\mathbb{C}^{*}}$ we have $s_{\eta, \mu}^{X} \geq s_{\eta}^{X}$, and if $s_{\eta, \mu}^{X}=s_{\eta}^{X}$ then $d_{\eta}^{X}$ is an upper bound for the logarithmic degeneracy of $s_{\eta, \mu}^{X}$. 
We postpone the proof of this theorem in the general case to the Appendix section 13. For the complex cases (i.e. when $X$ is a Riemannian symmetric space of type IV) the proof will be given below.

As an immediate consequence of theorem 7.1 we have

Theorem 7.2. We use the notation as introduced above. Given an extremal boundary point $\eta=\omega_{j} / k_{j}$ of $\Omega$, we consider $t_{\epsilon}=\exp (i \pi \eta / 2) \in A_{\mathbb{C}}$. Fix $-\pi<\theta_{1}<\theta_{2}<\pi$. Let $\mu \in \mathfrak{a}_{\mathbb{C}}{ }^{*}$, then there exist constants $r>0$, $C>0$, such that, for all $\epsilon \in S_{r, \theta_{1}, \theta_{2}}$,

$$
\left|\phi_{\mu}^{X}\left(t_{\epsilon}^{2} \cdot x_{0}\right)\right| \leq C \epsilon^{s_{\eta}^{X}}|\log (\epsilon)|^{d_{\eta}^{X}},
$$

where $s_{\eta}^{X}=s_{\eta}\left(m^{X}\right)$ and $d_{\eta}^{X}=d_{\eta}\left(m^{X}\right)$ for the functions $s_{\eta}$ and $d_{\eta}$ listed in Theorem 7.9.

For later applications it is useful to have a slightly weaker but more handy version of the estimate above. Let us define

$$
s^{X}:=\max _{\eta} s_{\eta}^{X} \text { and } d^{X}:=\max _{\eta: s_{\eta}^{X}=s^{X}} d_{\eta}^{X} .
$$

The theorem above combined with the maximum principle of holomorphic functions then yields

Theorem 7.3. For each $\mu \in \mathfrak{a}_{\mathbb{C}}{ }^{*}$ there exists a constant $C=C(\mu)>0$ such that for all $Y \in \partial \Omega$ and $0<\epsilon<1$

$$
\left|\phi_{\mu}^{X}\left(\exp (i(1-\epsilon) \pi Y) \cdot x_{0}\right)\right| \leq C \epsilon^{s^{X}}|\log (\epsilon)|^{d^{X}} .
$$

Since $\Xi$ only depends on the isogeny class of $G$ (Remark 2.3(c)) it suffices to do the analysis in the situation where $G_{\mathbb{C}}$ is simply connected. In addition we assume the restricted root system $\Sigma$ of $X$ to be irreducible. Recall that twice the character lattice of $A_{\mathbb{C}}$ is equal to the weight lattice of the restricted root system $\Sigma^{l}$. The categorical quotient $W \backslash A_{\mathbb{C}}$ (as well as $\left.W \backslash A_{\mathbb{C}} / F\right)$ is affine space.

There are two special cases which can be treated by direct methods, the real rank-one case and the complex case. It is both instructive and useful to consider these cases first before going to the general case which is treated in the Appendix 13.

7.0.1 The real rank one case. This case was treated in detail in [KrS1, Th. 5.1] but it is useful to discuss the difference between the approach used in [KrS1] and the approach in the present paper.

Let $G$ be a real semisimple group with real rank one. Then $\Sigma=\Sigma(\mathfrak{g}, \mathfrak{a})$ is of the form $\Sigma=\{ \pm \alpha\}$ (reduced case) or $\Sigma=\{ \pm \alpha / 2, \pm \alpha\}$ (non-reduced case). Let $\eta \in \mathfrak{a}$ be such that $\alpha(\eta)=1$, so that $\eta$ is at the boundary of $\Omega$. We put $q:=\operatorname{dim} \mathfrak{g}^{\alpha}$ and $p:=\operatorname{dim} \mathfrak{g}^{\alpha / 2}$ (so that $q \geq 1$, with $p=0$ (reduced 
case) or $p \geq q$ (non-reduced case); this is convenient but admittedly a bit unconventional in the reduced case).

In [KrS1] the Harish-Chandra integral representation for the spherical function $\phi_{\lambda}$ is analyzed directly to obtain the precise asymptotic behaviour of the holomorphic extension of $\phi_{\lambda}(\exp (i \pi(1-\epsilon) \eta))$ as $\epsilon \rightarrow 0$ if $\lambda \in i \mathfrak{a}^{*}$. The result of [KrS1] says that for $\lambda\left(\alpha^{\vee}\right) \in i \mathbb{R}$ one has

$$
\phi_{\lambda}(\exp (i \pi(1-\epsilon) \eta)) \asymp|\log \epsilon|
$$

if $q=1$, and

$$
\phi_{\lambda}(\exp (i \pi(1-\epsilon) \eta)) \asymp \epsilon^{1-q}
$$

if $q>1$.

The method of the present paper is not based on the analysis of the integral representation of the spherical function $\phi_{\lambda}$ but rather on the analysis of the radial system of eigenfunction equations for $\phi_{\lambda}$ with respect to the commutative algebra of $G_{\mathbb{C}}$-invariant differential operators on $\Xi$. In the case at hand, this amounts to the well-known fact that

$$
\phi_{\lambda}(\exp (i \pi(1-\epsilon) \eta))=F(a, b, c ; z),
$$

with

$$
\begin{aligned}
& a=\lambda\left(\alpha^{\vee}\right)+p / 4+q / 2, \\
& b=-\lambda\left(\alpha^{\vee}\right)+p / 4+q / 2, \\
& c=1 / 2+p / 2+q / 2, \\
& z=(1-\cos (\pi(1-\epsilon))) / 2=1-\pi^{2} \epsilon^{2} / 4+\ldots
\end{aligned}
$$

where $F(a, b, c ; z)$ (with $a, b, c \in \mathbb{C},|z|<1$ ) denotes the Gauss hypergeometric function. This function is the unique holomorphic solution of the hypergeometric differential equation

$$
z(1-z) \frac{d^{2} F}{d^{2} z}+(c-(a+b+1) z) \frac{d F}{d z}-a b F=0
$$

on the unit disc, normalized by $F(a, b, c ; 0)=1$. The hypergeometric differential equation is a second order Fuchsian equation with regular singularities at $z=0, z=1$ and $z=\infty$. The exponents of (7.9) at $z=1$ are easily seen to be equal to 0 and $c-a-b=1 / 2(1-q)$. Hence the problem of finding the singular expansion of $\phi_{\lambda}(\exp (i \pi(1-\epsilon) \eta))$ at $\epsilon=0$ is now transformed to a simple exercise on the analytic continuation of the solutions of (7.9). If $\phi_{\lambda}(\exp (i \pi(1-\epsilon) \eta))=F(a, b, c ; z)$ is holomorphic at $z=1$ (i.e. at $\epsilon=0)$ then $F(a, b, c ; z)$ extends holomorphically to $\mathbb{C}$. Since $\infty$ is a regular singular point of (7.9) this implies that $F(a, b, c ; z)$ is a polynomial in $z$ in this case (essentially a so-called Jacobi polynomial). The hypergeometric series terminates iff $a$ or $b$ is a non-positive integer (since $c$ is not a negative 
integer in our situation), i.e. iff $\lambda\left(\alpha^{\vee}\right)= \pm(p / 4+q / 2+n)$ with $n \in \mathbb{Z}_{\geq 0}$. Hence for these values of $\lambda$ the function $\phi_{\lambda}(\exp (i \pi(1-\epsilon) \eta))$ is asymptotic to a constant (for $\epsilon \rightarrow 0$ ), and for all other values of $\lambda$ we find (7.6) or (7.7) (whichever is relevant).

In the general case we need to deal with Harish-Chandra's radial system of eigenfunction equations for the $G$-invariant differential operators, which is a holonomic system of differential equations on $W \backslash A_{\mathbb{C}} / F$ with regular singularities along the discriminant locus. This gives rise to various questions and problems which are addressed in the Appendix 13. First of all it is not clear in general how to define a set of singular exponents at a point of the singular locus of a regular singular holonomic system. In subsection 13.1 we give an appropriate definition of the notion of exponents in the special case of a holonomic system of differential equations with regular singularities along an affine hyperplane arrangement. We apply this definition to the pull back to $\mathfrak{a}_{\mathbb{C}}$ of the Harish-Chandra system of equations. The next problem is the actual computation of the set of exponents at $\eta$. We do not know how to do this directly in an algebraic way in general; instead we use the relation between the exponents and monodromy of the solutions of the system of equations and, in a crucial way, the parameter deformation of the Harish-Chandra system provided by the so-called hypergeometric system of differential equations for root systems. Via the monodromy representation affine Hecke algebras play a role in dealing with these computations. A final problem in the higher rank case is the selection of those exponents among the full set of exponents at $\eta$ which may be involved in the expansion of $\phi_{\lambda}$ at $\eta$, and among those, the leading exponent. Again some basic representation theory of the affine Hecke algebra plays an important role at this stage. The outcome is remarkable; the leading exponent is attached to a specific irreducible character of the isotropy group $W_{\eta}^{a}$ of $\eta$ in the affine Weyl group $W^{a}$. We call this irreducible character the leading character at $\eta$. It turns out that the leading character (for generic $\lambda$ ) depends on the local geometry of $\Omega$ at $\eta$, but not on the root multiplicities.

7.0.2 The complex case. We consider the complex case $X=G / U$ where $G$ is the connected simply connected complex simple group and $U$ its maximal compact subgroup. As is well known, by the work of HarishChandra [H1] the spherical functions are of an elementary nature in this case, allowing us to analyze the desired exponents directly. The restricted root system $\Sigma$ of $X$ is equal to twice the root system of $G$, and all root multiplicities are equal to 2 . We introduce the Weyl denominator $\delta$ on 
$A_{\mathbb{C}} / F$ by

$$
\delta(a)=\prod_{\alpha \in \Sigma_{+}}\left(\alpha(a)-\alpha(a)^{-1}\right),
$$

and we denote by $A_{\mathbb{C}}{ }^{\text {reg }} / F$ the complement of the set $\delta=0$ in $A_{\mathbb{C}} / F$. The algebra $\mathcal{R}_{X}$ of radial parts of invariant differential operators on $X$ consists of the differential operators on $A_{\mathbb{C}}{ }^{\text {reg }} / F$ of the form

$$
\mathcal{R}_{X}=\left\{\delta^{-1} \circ \partial(p) \circ \delta \mid p \in \mathbb{C}\left[\mathfrak{a}^{*}\right]^{W}\right\} .
$$

The Harish-Chandra isomorphism $\gamma_{X}: \mathcal{R}_{X} \rightarrow \mathbb{C}\left[\mathfrak{a}^{*}\right]^{W}$ is given by $\gamma_{X}\left(\delta^{-1}\right.$ 。 $\partial(p) \circ \delta)=\partial(p)$. Now $\left.\phi_{\mu}^{X}\right|_{A_{\mathbb{C}}}$ satisfies the following system of eigenfunction equations

$$
D \phi=\gamma_{X}(D)(\mu) \phi, \quad \forall D \in \mathcal{R}_{X} .
$$

This is a $W$-equivariant system of differential equations on $A_{\mathbb{C}}{ }^{\text {reg }} / F$. It is also equivariant for the action of the 2-group $F=A_{\mathbb{C}} \cap U$. Therefore we can view (7.12) as a system of differential equations on $W \backslash A_{\mathbb{C}} / F-\{d=0\}$, where $d=\delta^{2}$ is the discriminant of $W$, viewed as a polynomial on $W \backslash A_{\mathbb{C}} / F$. We call $\mathcal{L}_{X}$ the sheaf of local solutions of (7.12).

A general local solution to this set of equations is of the form

$$
\phi=\delta^{-1} \psi
$$

where $\psi$ is a local solution of the constant coefficient system

$$
\partial(p) \psi=p(\mu) \psi, \quad \forall p \in \mathbb{C}\left[\mathfrak{a}^{*}\right]^{W} .
$$

Let $\exp : \mathfrak{a}_{\mathbb{C}} \rightarrow A_{\mathbb{C}}$ denote the exponential map such that $\exp (2 \pi i X)=1$ iff $X \in Q\left(\Sigma^{\vee}\right)$. Consider the covering map

$$
\pi: \mathfrak{a}_{\mathbb{C}}{ }^{\text {reg }} \rightarrow W \backslash A_{\mathbb{C}}{ }^{\text {reg }} / F
$$

where $\mathfrak{a}_{\mathbb{C}}{ }^{\text {reg }}$ is the complement in $\mathfrak{a}_{\mathbb{C}}$ of the set of affine root hyperplanes, the zero sets of the affine roots $a=\alpha-n$ (with $\alpha \in \Sigma$ and $n \in \mathbb{Z}$ ), and $\pi$ is given by $\pi(X)=W \exp (\pi i X) F$. The following proposition is well known.

Proposition 7.4. The space of solutions of (7.12) on a nonempty open ball $U \subset W \backslash A_{\mathbb{C}}{ }^{\text {reg }} / F$ consists of holomorphic functions and has dimension $|W|$ (independent of $\mu$ ). Let $V \subset \pi^{-1}(U)$ be a connected component. The pull back of a local solution of (7.12) on $U$ via $\left.\pi\right|_{V}$ extends to a global holomorphic function on $\mathfrak{a}_{\mathbb{C}}{ }^{\text {reg }}$.

Proof. We use the general form (7.13) of the local solutions. By a wellknown result of Steinberg [St1] the global solution space of (7.14) on $\mathfrak{a}_{\mathbb{C}}$ has dimension equal to $|W|$ (independent of $\mu$ ) and consists of entire functions. On the other hand, the left ideal in the ring of differential operators with holomorphic coefficients on $\mathfrak{a}_{\mathbb{C}}$ generated by the operators $\partial(p)-p(\lambda)$ (with $p \in \mathbb{C}\left[\mathfrak{a}^{*}\right]^{W}$ ) is cofinite, a complement being generated by constant 
coefficient operators $\partial(q)$ where $q$ is running over a set of polynomials representing a basis of the coinvariant algebra. Hence the local solution space is at most of dimension $|W|$. The proposition follows.

Corollary 7.5. The system (7.12) is holonomic of rank $|W|$. Upon choosing a base point $p \in \mathfrak{a}_{\mathbb{C}}{ }^{\text {reg }}$ we may view the monodromy representation as a representation of the group of deck transformations of the covering map $\pi$, which is the affine Weyl group $W^{a}=W \ltimes Q\left(\Sigma^{\vee}\right)$ acting on $\mathfrak{a}_{\mathbb{C}}$. The restriction of the monodromy representation to $W$ is equivalent to the regular representation.

Proof. All is clear except for the last assertion. By equation (7.13) it is enough to know this for the space of solutions of (7.14). This is well known, and follows from the case $\mu \in \mathfrak{a}_{\mathbb{C}}{ }^{\text {reg }}$ by rigidity of characters of a finite group.

Notice that the center of the group ring of $W^{a}$ is $\mathbb{C}\left[Q\left(\Sigma^{\vee}\right)\right]^{W}$. Thus the central characters of irreducible representations of $W^{a}$ correspond to $W$-orbits of points of the complex algebraic torus

$$
T^{L}=\mathfrak{a}_{\mathbb{C}}{ }^{*} / P(\Sigma)
$$

whose exponential map we will denote by $\exp ^{L}$ (i.e. $\exp ^{L}(\mu)=1$ iff $\mu \in P(\Sigma)$ ). Then $T^{L}$ is the dual torus of $A_{\mathbb{C}}$.

Proposition 7.6. The monodromy representation of (7.12) has central character $W \exp ^{L}(\mu) \in W \backslash T^{L}$ (observe that this central character is unitary iff $\mu \in \mathfrak{a}^{*}$ is real). The monodromy representation is irreducible iff $\exp ^{L}(\mu)$ has trivial isotropy for the action of $W$ on $T^{L}$.

Proof. The monodromy representation clearly has central character $W \exp ^{L} \mu$, and dimension $|W|$ by Proposition 7.4. The last assertion follows easily from the Mackey induction procedure.

The spherical function $\left.\phi_{\mu}^{X}\right|_{A_{\mathbb{C}}}$ is a special solution of (7.12), which can be characterized by saying that it is a nonzero $W$-fixed vector in the monodromy representation. By the above corollary the space of $W$-fixed vectors is one-dimensional. Looking at (7.13) we see that $\left.\phi_{\mu}^{X}\right|_{A_{\mathbb{C}}}$ is of the form $\delta^{-1} \psi$ where $\psi$ is a $W$-skew solution (say on $\mathfrak{a}_{\mathbb{C}}$ ) of (7.14). Then $\psi$ is divisible by $\prod_{\alpha \in \Sigma_{+}} \alpha$, and thus $\left.\phi_{\mu}^{X}\right|_{A_{\mathbb{C}}}$ extends to a holomorphic solution on $A T_{\Omega}^{2}$.

We continue the discussion by considering $\left.\phi_{\mu}^{X}\right|_{A_{\mathbb{C}}}$ via $\pi$ as a holomorphic function on $i \mathfrak{a}+\Omega$ of the form $\delta^{-1} \psi$ with $\psi$ a $W$-skew solution of (7.14). Let $\eta=\omega_{i} / k_{i}$ be as before. We denote by $W_{\eta}$ the isotropy group of $\eta$ in $W$, and by $W_{\eta}^{a}$ the isotropy group of $\eta$ in the affine Weyl group $W^{a}$. 
Lemma 7.7. The natural homomorphism $W^{a} \rightarrow W$ restricts to an isomorphism from $W_{\eta}^{a}$ onto the isotropy group $W_{t(\eta)^{2} F} \subset W$ of $t(\eta)^{2} F=$ $\exp (i \pi \eta) F$ for the action of $W$ on $A_{\mathbb{C}} / F$. In particular $W_{t(\eta)^{2} F}=W$ if $\eta \in \partial(\Omega)$ is minuscule.

Proof. The injectivity is clear. The isomorphism of complex algebraic tori $A_{\mathbb{C}} / F \approx A_{\mathbb{C}}$ given by $a F \rightarrow a^{2}$ is $W$-equivariant. Therefore $W_{t(\eta)^{2} F}$ is equal to the isotropy group of $W_{t(\eta)^{4}}$ for the action of $W$ on $A_{\mathbb{C}}$. Since $A_{\mathbb{C}}$ has the weight lattice $P(\Sigma)$ as its character lattice the group $W_{t(\eta)^{4}}$ is generated by reflections (by a well-known result of Steinberg [St2]). Now suppose that $s=s_{\alpha} \in W_{t(\eta)^{4}}$ is a reflection. But $s \in W_{t(\eta)^{4}} \Longleftrightarrow s(\eta)-\eta \in Q\left(\Sigma^{\vee}\right) \Longleftrightarrow$ $\alpha(\eta)=n \in \mathbb{Z}$. Hence the affine reflection $s_{\alpha-n}$ satisfies $s_{\alpha-n} \in W_{\eta}^{a}$ and is mapped to $s$, proving the surjectivity.

Let $\Sigma_{+, \eta}^{a}$ be the set of positive affine roots which vanish on $\eta$, and let $\Sigma_{+, \eta}=\Sigma_{+, \eta}^{a} \cap \Sigma$. Then the Dynkin diagram of $\Sigma_{+, \eta}^{a}$ is $D^{*}-\left\{\alpha_{\eta}\right\}$ where $\alpha_{\eta}$ is the unique simple root of $\Sigma$ such that $\alpha_{\eta}(\eta) \neq 0$, and $\Sigma_{\eta} \subset \Sigma_{\eta}^{a}$ is the maximal standard parabolic subsystem in which we delete $\alpha_{0}$ from the set of simple roots of $\Sigma_{\eta}^{a}$.

In a small neighborhood of the extremal boundary point $\eta$ of $\Omega$ we consider the Taylor expansion of $\psi$. The lowest homogeneous term $h_{\eta, \mu}$ of $\psi$ at $\eta$ is a $W$-harmonic polynomial which is $W_{\eta}$ skew, and $\left.\phi_{\mu}^{X}\right|_{A_{\mathbb{C}}} \circ \pi$ can be uniquely expressed in the form

$$
\left.\phi_{\mu}^{X}\right|_{A_{\mathbb{C}}} \circ \pi=\left(\prod_{a \in \Sigma_{\eta,+}^{a}} a\right)^{-1}\left(h_{\eta, \mu}+\text { higher order terms at } \eta\right) .
$$

Since $h_{\eta, \mu}$ is divisible by $\prod_{\alpha \in \Sigma_{\eta,+}} \alpha$ we see that in this case the leading exponent $s_{\eta, \mu}^{X}$ satisfies

$$
s_{\eta, \mu}^{X} \geq s_{\eta}^{X}:=-\left|\Sigma_{\eta,+}^{a}-\Sigma_{\eta,+}\right| .
$$

For $\mu$ in an open, dense subset of $\mathfrak{a}_{\mathbb{C}}{ }^{*}$ this bound is sharp. The bound is sharp if $\mu=i \lambda$ with $\lambda \in \mathfrak{a}^{*}$. It is not so easy to describe the function $\mu \rightarrow s_{\eta, \mu}^{X}$ exactly. We observe that this function is upper semi-continuous.

The above analysis cannot be used directly in general, since the spherical functions do not have a simple factorization formula like (7.13) in general. For our later use it is helpful to describe the above result in terms of the monodromy representation. By the above we see that $h_{\eta, \mu}$ belongs to space of $W$-harmonic polynomials which transform by the sign representation under the action of $W_{\eta}$. This means that $h_{\eta}$ is a $W$-harmonic polynomial in the direct sum of the isotypical components of the irreducible characters of $W_{\eta}^{a}$ which are induced from the sign representation $\operatorname{det}_{\eta}$ of $W_{\eta}$. Therefore 
the homogeneous degree of $h_{\eta}$ at $\eta$ is at least equal to the harmonic birthday of the irreducible character (the leading character) $\tilde{\sigma}_{\eta} \in \operatorname{Irr}\left(W_{\eta}^{a}\right)$ given by the truncated induction

$$
\tilde{\sigma}_{\eta} \in \operatorname{Irr}\left(W_{\eta}^{a}\right)=j_{W_{\eta}}^{W_{\eta}^{a}}\left(\operatorname{det}_{\eta}\right)
$$

It follows by truncated induction (see $[\mathrm{C}, \S 11.2]$ ) that the harmonic birthday of this irreducible character is equal to $\left|\Sigma_{\eta,+}\right|$, and that this representation has multiplicity 1 in this degree. Moreover, the same is true in the space of $W$-harmonic polynomials.

Proposition 7.8. Assume we are in the complex case, so $X$ is a Riemannian symmetric space of type $I V$ with restricted root system $\Sigma$. Let $\eta \in \partial_{e} \Omega \cap \mathbb{C}$ be an extremal boundary point of $\Omega$ as before, and define $-s_{\eta}^{X}:=\left|\Sigma_{\eta,+}^{a}-\Sigma_{\eta,+}\right|$ as the number of roots $\alpha$ in $\Sigma_{+}$with $\alpha(\eta)=1$. Let $s_{\eta, \mu}^{X}$ be the leading exponent at $z=t(\eta)^{2} \cdot x_{0}$ in the sense of (7.2). For all $\mu \in \mathfrak{a}_{\mathbb{C}}{ }^{*}$ the logarithmic degeneracy of the leading exponent $s_{\eta, \mu}^{X}$ is 0 . For all $\mu \in \mathfrak{a}_{\mathbb{C}}{ }^{*}$ we have $s_{\eta, \mu}^{X} \geq s_{\eta}^{X}$. For $\mu$ in a dense open set of $\mathfrak{a}_{\mathbb{C}}{ }^{*}$ containing $i \mathfrak{a}^{*}$ this inequality is an equality (cf. Theorem 7.9).

Theorem 7.9. In Table 4 below we have used the numbering of the extremal boundary points $\eta_{j}=\omega_{j} / k_{j} \in \partial \Omega$ corresponding to the distinguished boundary orbits as in Table 1. Table 4 displays lower bounds for the leading exponents of the holomorphically extended elementary spherical functions at the extremal points $\eta_{j}$ in the sense of (7.2). In the case where this lower bound is attained the table displays the corresponding logarithmic degeneracy and a leading character (leading characters are explained in Appendix 13).

The convention for the root multiplicities is as follows. We use $m_{1} \geq 1$ for the root multiplicity of a long root $\alpha$ (or simply $m$ if $\Sigma$ is reduced and simply laced). The multiplicity of half a long root in $\Sigma$ is denoted by $m_{1 / 2} \geq 0$ (i.e. we view $C_{n}$ as the special case of $B C_{n}$ where $m_{1 / 2}=0$ ). The multiplicity of unmultipliable roots $\beta \in \Sigma$ (i.e. $2 \beta \notin \Sigma$ ) which are not long roots is denoted by $m_{2} \geq 1$ (if such roots exist).

The proof Table 4 and these facts is given in the Appendix in section 13. In the parameter family of hypergeometric functions $\phi_{\mu, m}$ (cf. Appendix 13) with real multiplicities $m$ the indicated lower bounds are sharp generically in $m$, provided that $m$ satisfies the inequalities $1 \leq m_{1} \leq m_{2}$. The leading character is independent of $m$ in this cone (hence only depends on the geometry of $\Omega$ at the extremal point $\eta$ ). 


\begin{tabular}{|c|c|c|c|c|c|}
\hline \multicolumn{6}{|c|}{ The leading character $\sigma_{\eta} \in \operatorname{Irr} W_{\eta}^{a}$, the leading exponent $s_{\eta}$ and its degeneracy $d_{\eta}$} \\
\hline $\bar{\Sigma}$ & $\eta$ & $\overline{\Sigma_{\eta}^{a}}$ & $\sigma_{\eta}$ & $s_{\eta}$ & $d_{\eta}=1$ iff \\
\hline$A_{2 r}(r \geq 1)$ & $\omega_{j}(j \leq r)$ & $A_{2 r}$ & $\overline{(2 r-j+1, j)}$ & $j(1-(2 r+2-j) m / 2)$ & \\
\hline$A_{2 r-1}(r \geq 2)$ & $\omega_{j}(j \leq r)$ & $A_{2 r-1}$ & $(2 r-j, j)$ & $j(1-(2 r+1-j) m / 2)$ & $m=1 \& j=r$ \\
\hline$B_{l}(l \geq 3)$ & $\omega_{1}$ & $\overline{B_{l}}$ & $(l-1,1)$ & $1-(l-1) m_{1}-m_{2}$ & \\
\hline$B_{3}$ & $\overline{\omega_{3} / 2}$ & $A_{3}$ & $(3,1)$ & $1-2 m_{1}$ & \\
\hline$B_{2 r}(r \geq 2)$ & $\omega_{2 r} / 2$ & $D_{2 r}$ & $(r, r)$ & $r\left(1-r m_{1}\right)$ & \\
\hline$B_{2 r+1}(r \geq 2)$ & $\omega_{2 r+1} / 2$ & $D_{2 r+1}$ & $(r+1, r)$ & $r\left(1-(r+1) m_{1}\right)$ & \\
\hline$B C_{1}$ & $\omega_{1}$ & $\overline{A_{1}}$ & 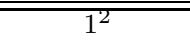 & c1-m & $\overline{m_{1}=1}$ \\
\hline$B C_{2 r}(r \geq 1)$ & $\omega_{2 r}$ & $C_{2 r}$ & $(r, r)$ & $r\left(1-r m_{2}-m_{1}\right)$ & \\
\hline$B C_{2 r+1}(r \geq 1)$ & $\omega_{2 r+1}$ & $C_{2 r+1}$ & $(r, r+1)$ & $(r+1)\left(1-r m_{2}-m_{1}\right)$ & $m_{1}=1$ \\
\hline$\overline{D_{l}(l \geq 4)}$ & $\overline{\omega_{1}}$ & $\overline{D_{l}}$ & $(((l-1,1),-)$ & $2-\operatorname{lm}$ & $\bar{m} m=1$ \\
\hline$D_{2 r}(r \geq 2)$ & $\omega_{2 r}$ & $\overline{D_{2 r}}$ & $(r, r)^{\prime}$ & $r(1-r m)$ & \\
\hline$D_{2 r+1}(r \geq 2)$ & $\omega_{2 r+1}$ & $D_{2 r+1}$ & $(r+1, r)$ & $r(1-(r+1) m)$ & \\
\hline 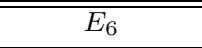 & $\omega_{1}$ & $\bar{E}_{6}$ & $\phi_{20,2}$ & $2-9 m$ & \\
\hline$E_{7}$ & $\omega_{7}$ & $E_{7}$ & $\phi_{21,3}$ & $3-15 m$ & $m=1$ \\
\hline & $\omega_{2} / 2$ & $A_{7}$ & $(7,1)$ & $1-4 m$ & \\
\hline$E_{8}$ & 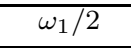 & $D_{8}$ & $((7,1),-)$ & $2-8 m$ & $m=1$ \\
\hline & $\omega_{2} / 3$ & $A_{8}$ & $(8,1)$ & $1-9 / 2 m$ & \\
\hline$F_{4}$ & $\omega_{4} / 2$ & $B_{4}$ & $\overline{(3,1)}$ & $1-3 m_{1}-m_{2}$ & \\
\hline$G_{2}$ & $\overline{\omega_{1} / 3}$ & $A_{2}$ & $(2,1)$ & $1-3 / 2 m_{1}$ & \\
\hline
\end{tabular}

Table 4

\section{Unipotent Model for the Crown Domain}

In this section we give a new geometrical characterization of the crown by unipotent $G$-orbits. For the beginning there is no restriction on $G$ and we define a connected $G$-subset of $X_{\mathbb{C}}$ by

$$
\Xi_{N}=G \exp (i \mathfrak{n}) \cdot x_{0}=G N_{\mathbb{C}} \cdot x_{0} .
$$

For $G=\operatorname{Sl}(2, \mathbb{R})$ we have shown that $\Xi_{N}$ is an open subset, but in the general case this is not clear to us. The next lemma contains the crucial information.

Lemma 8.1. $\quad \Xi \subset \Xi_{N}$.

Proof. Let $Y \in \pi \Omega / 2$. We recall the complex convexity theorem (5.4)

$$
\operatorname{Im} \log a_{\mathbb{C}}\left(K \exp (i Y) \cdot x_{0}\right)=\operatorname{co}(W . Y) .
$$

In particular, there exists a $k \in K$ such that $\operatorname{Im} \log a_{\mathbb{C}}\left(k \exp (i Y) \cdot x_{0}\right)=0$, or, in other words,

$$
k \exp (i Y) \in N_{\mathbb{C}} A K_{\mathbb{C}}=A N_{\mathbb{C}} K_{\mathbb{C}} .
$$


We conclude that $G \exp (i Y) \subset G N_{\mathbb{C}} K_{\mathbb{C}}$ and then $G \exp (i \Omega) \subset G N_{\mathbb{C}} K_{\mathbb{C}}$, i.e. $\Xi \subset \Xi_{N}$.

Let us define a domain $\Lambda \subset \mathfrak{n}$ by

$$
\Lambda=\left\{Y \in \mathfrak{n} \mid \exp (i Y) \cdot x_{0} \in \Xi\right\}_{0}
$$

where $\{\cdot\}_{0}$ stands for the connected component of $\{\cdot\}$ containing 0 . As $\Xi$ is open, it is clear that $\Lambda$ is open as well.

Lemma 8.2. Suppose that $\Omega_{c} \subset \Omega$ is a compact subset. Then the set

$$
\Lambda_{c}=\left\{Y \in \mathfrak{n} \mid \exp (i Y) \cdot x_{0} \in G \exp \left(i \Omega_{c}\right) \cdot x_{0}\right\}
$$

is compact in $\mathfrak{n}$.

Proof. First we observe that $\Lambda_{c}$ is closed as $G \exp \left(i \Omega_{c}\right) \cdot x_{0}$ is closed in $X_{\mathbb{C}}$.

Let $Y \in \Lambda_{c}$. Then $\exp (i Y)=g \exp (i Z) \cdot x_{0}$ for some $g \in G$ and $Z \in \Omega_{c}$. With $g=n^{-1} a^{-1} k$ for $n \in N, a \in A$ and $k \in K$ we obtain that

$$
k \exp (i Z) \cdot x_{0}=\text { an } \exp (i Y) \cdot x_{0} .
$$

We recall that $\Xi \subset A_{\mathbb{C}} N_{\mathbb{C}} \cdot x_{0}$ and that there is a well-defined holomorphic projection

$$
\tilde{n}: A_{\mathbb{C}} N_{\mathbb{C}} \cdot x_{0} \rightarrow N_{\mathbb{C}} .
$$

Further we note that the map

$$
N \times \mathfrak{n} \rightarrow N_{\mathbb{C}}, \quad(n, Y) \mapsto n \exp (i Y)
$$

is a diffeomorphism. In particular $N \backslash N_{\mathbb{C}} \simeq \mathfrak{n}$ under a homeomorphic map $\psi$. Consider the continuous map

$$
f=\psi \circ \tilde{n}: A_{\mathbb{C}} N_{\mathbb{C}} \cdot x_{0} \rightarrow \mathfrak{n},
$$

and note that (8.1) shows that $f\left(k \exp (i Z) \cdot x_{0}\right)=Y$. Therefore

$$
\Lambda_{c} \subset f\left(K \exp \left(i \Omega_{c}\right) \cdot x_{0}\right) .
$$

Since $K \exp \left(i \Omega_{c}\right) \cdot x_{0}$ is compact and $f$ is continuous, the assertion of the lemma follows.

We arrive at the main result of this section.

Theorem 8.3. $\Xi=G \exp (i \Lambda) \cdot x_{0}$.

Proof. We argue by contradiction. Suppose that the assertion is false. Then there exists $Z \in \Omega$ such that $\exp (i Z) \cdot x_{0} \notin G \exp (i \Lambda) \cdot x_{0}$ and sequences $\left(Z_{n}\right)_{n \in \mathbb{N}} \subset \Omega,\left(g_{n}\right)_{n \in \mathbb{N}} \subset G$ and $\left(Y_{n}\right)_{n \in \mathbb{N}} \subset \Lambda$ such that $Z_{n} \rightarrow Z$ and $\exp \left(i Z_{n}\right) \cdot x_{0}=g_{n} \exp \left(i Y_{n}\right) \cdot x_{0}$. Let $\Omega_{c} \subset \Omega$ be a compact subset of $\Omega$ with $\left(Z_{n}\right)_{n \in \mathbb{N}} \subset \Omega_{c}$. Then $\exp \left(i Y_{n}\right) \cdot x_{0} \in G \exp \left(i \Omega_{c}\right) \cdot x_{0}$ and we conclude from Lemma 8.2 that $\left(Y_{n}\right)_{n \in \mathbb{N}}$ is a bounded sequence in $\mathfrak{n}$. W.l.o.g. we may assume that $Y_{n} \rightarrow Y \in \mathfrak{n}$. As $\Omega_{c}$ is compact, the set $G \exp \left(i \Omega_{c}\right) \cdot x_{0}$ is closed in $X_{\mathbb{C}}$ (cf. Remark 2.2) and thus $\exp (i Y) \cdot x_{0} \in G \exp \left(i \Omega_{c}\right) \cdot x_{0} \subset \Xi$. Hence 
$Y \in \Lambda$. Because $G$ acts properly on $\Xi$ we conclude that $\left(g_{n}\right)_{n \in \mathbb{N}}$ is bounded and it is no loss of generality to assume that $\lim _{n \rightarrow \infty} g_{n}=g$. But then $\exp (i Z) \cdot x_{0}=g \exp (i Y) \cdot x_{0} \in G \exp (i \Lambda) \cdot x_{0}$, a contradiction.

REMARK 8.4. The determination of the precise shape of $\Lambda$ is a difficult problem, especially for higher rank groups. Generally one might ask: Is $\Lambda$ always bounded? Is $\Lambda$ convex?

Recall the fact that $G \exp (i Z) \cdot x_{0}=G \exp \left(i Z^{\prime}\right) \cdot x_{0}$ for $Z, Z^{\prime} \in \pi \Omega / 2$ means that $W \cdot Z=W \cdot Z^{\prime}$. Thus we obtain a well-defined map

$$
p: \Lambda \rightarrow \Omega / W
$$

via $G \exp (i Y) \cdot x_{0}=G \exp (i \pi p(Y) / 2) \cdot x_{0}$ for $Y \in \Lambda$. The following would be interesting to know: What are the fibers of the map $p$ ? What are the preimages of the extreme points? Is there an expressible relationship between $Y$ and $p(Y)$ ?

8.1 The case of real rank one. In this subsection we will determine the precise shape of $\Lambda$ for groups $G$ with real rank one. We begin with a criterion which is useful for later computations.

Lemma 8.5. Suppose that $G$ has real rank one. Then

$$
\Lambda=\left\{Y \in \mathfrak{n} \mid N \exp (i Y) \cdot x_{0} \subset \bar{N}_{\mathbb{C}} A_{\mathbb{C}} \cdot x_{0}\right\}_{0}
$$

with $\{\cdot\}_{0}$ denoting the connected component of $\{\cdot\}$ containing 0 .

Proof. Set

$$
\Lambda_{1}=\left\{Y \in \mathfrak{n} \mid N \exp (i Y) \cdot x_{0} \subset \bar{N}_{\mathbb{C}} A_{\mathbb{C}} \cdot x_{0}\right\}_{0},
$$

and note that

$$
\Lambda_{1}=\left\{Y \in \mathfrak{n} \mid \exp (i Y) \cdot x_{0} \subset \bigcap_{n \in N} n \bar{N}_{\mathbb{C}} A_{\mathbb{C}} \cdot x_{0}\right\}_{0} .
$$

We recall the fundamental fact on (general) complex crowns that

$$
\Xi=\left[\bigcap_{g \in G} g \bar{N}_{\mathbb{C}} A_{\mathbb{C}} \cdot x_{0}\right]_{0}
$$

with $[\cdot]_{0}$ denoting the connected component of $[$,$] containing x_{0}$. We are now going to use the fact that $G$ has real rank one. In particular $W=$ $\{1, w\}=\mathbb{Z}_{2}$ and the Bruhat decomposition of $G$ reads $G=N M A \bar{N} \cup w M A \bar{N}$. Hence

$$
\Xi=\left[\bigcap_{n \in N} n \bar{N}_{\mathbb{C}} A_{\mathbb{C}} \cdot x_{0} \cap N_{\mathbb{C}} A_{\mathbb{C}} \cdot x_{0}\right]_{0} .
$$

As a result (8.2) translates into

$$
\Lambda_{1}=\{Y \in \mathfrak{n} \mid \exp (i Y) \in \Xi\}_{0}=\Lambda,
$$

and the proof is complete. 
We introduce coordinates on $\mathfrak{n}=\mathfrak{g}^{\alpha}+\mathfrak{g}^{2 \alpha}$. As usual we write $p=\operatorname{dim} \mathfrak{g}^{\alpha}$ and $q=\operatorname{dim} \mathfrak{g}^{2 \alpha}$ and let $c=\frac{1}{4(p+4 q)}$. We endow $\mathfrak{n}$ with the inner product $\left\langle Y_{1}, Y_{2}\right\rangle=-\kappa\left(Y_{1}, \theta\left(Y_{2}\right)\right)$ where $\kappa$ denotes the Cartan-Killing form of $\mathfrak{g}$.

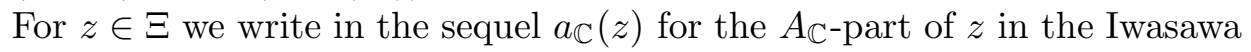
decomposition $\bar{N}_{\mathbb{C}} A_{\mathbb{C}} \cdot x_{0}$. For $Y \in \mathfrak{g}^{\alpha}, Z \in \mathfrak{g}^{2 \alpha}$ we recall the formula

$$
a_{\mathbb{C}}\left(\exp (Y+Z) \cdot x_{0}\right)^{\rho}=\left[\left(1+c\|Y\|^{2}\right)^{2}+4 c\|Z\|^{2}\right]^{\frac{p+2 q}{4}} .
$$

The complex linear extension of $\langle\cdot, \cdot\rangle$ to $\mathfrak{n}_{\mathbb{C}}$ shall be denoted by the same symbol. We obtain the following criterion for $\Lambda$.

LEMma 8.6. If $G$ has real rank one, then

$$
\begin{aligned}
\Lambda=\{(Y, Z) & \in \mathfrak{g}^{\alpha} \oplus \mathfrak{g}^{2 \alpha} \mid\left(\forall\left(Y^{\prime}, Z^{\prime}\right) \in \mathfrak{n}\right) \quad\left(1+c\left\langle Y^{\prime}+i Y, Y^{\prime}+i Y\right\rangle\right)^{2} \\
& \left.+4 c\left\langle Z^{\prime}+i Z+i 1 / 2\left[Y^{\prime}, Y\right], Z^{\prime}+i Z+i 1 / 2\left[Y^{\prime}, Y\right]\right\rangle \neq 0\right\}_{0} .
\end{aligned}
$$

Proof. A standard argument (see [KrS1, Lem.1.6]) combined with Lemma 8.5 yields that

$$
\Lambda=\left\{Y \in \mathfrak{n} \mid(\forall n \in N) a_{\mathbb{C}}(n \exp (i Y)) \text { is defined }\right\}_{0} .
$$

Now for $n=\exp \left(Y^{\prime}+Z^{\prime}\right) \in N$ with $\left(Y^{\prime}, Z^{\prime}\right) \in \mathfrak{n}$ and $(Y, Z) \in \Lambda$ one has

$$
n \exp (i(Y+Z))=\exp \left(Y^{\prime}+i Y+Z^{\prime}+i\left(Z+1 / 2\left[Y^{\prime}, Y\right]\right)\right),
$$

and the assertion follows in view of the explicit formula (8.3).

We use the criterion in Lemma 8.6 to determine $\Lambda$ explicitly. However, this is not so easy as it looks in the beginning. We shall begin with two important special cases and start with the Lorentz groups $G=\mathrm{SO}_{e}(1, p+1)$ where $q=0$.

Lemma 8.7. Assume that $G$ is locally $\mathrm{SO}_{e}(1, p+1)$. Then $c=1 / 4 p$ and

$$
\Lambda=\left\{Y \in \mathfrak{n}=\mathbb{R}^{p} \mid c\|Y\|^{2}<1\right\} .
$$

Proof. In view of the previous lemma we have to look at the connected component of those $Y \in \mathfrak{n}$ such that

$$
1+c\left\langle Y^{\prime}+i Y, Y^{\prime}+i Y\right\rangle=1+c\left(\left\|Y^{\prime}\right\|^{2}-\|Y\|^{2}-2 i\left\langle Y, Y^{\prime}\right\rangle\right) \neq 0
$$

for all $Y^{\prime} \in \mathfrak{n}$. The assertion follows.

Next we consider the case of the group $G=\mathrm{SU}(2,1)$. Here $p=2$ and $q=1$ and so $c=1 / 24$. Define matrix elements

$$
X_{\alpha}=\left(\begin{array}{ccc}
0 & 1 & 0 \\
-1 & 0 & 1 \\
0 & 1 & 0
\end{array}\right), \quad Y_{\alpha}=\left(\begin{array}{ccc}
0 & i & 0 \\
i & 0 & -i \\
0 & i & 0
\end{array}\right), \quad X_{2 \alpha}=\frac{1}{2}\left(\begin{array}{ccc}
i & 0 & -i \\
0 & 0 & 0 \\
i & 0 & -i
\end{array}\right)
$$

and note that

$$
\mathfrak{g}^{\alpha}=\mathbb{R} X_{\alpha} \oplus \mathbb{R} Y_{\alpha} \quad \text { and } \quad \mathfrak{g}^{2 \alpha}=\mathbb{R} X_{2 \alpha}
$$


We record the commutator relation $\left[X_{\alpha}, Y_{\alpha}\right]=4 X_{2 \alpha}$ and the orthogonality relation $\left\langle X_{\alpha}, Y_{\alpha}\right\rangle=0$. Finally we need that $\left\|X_{\alpha}\right\|^{2}=\left\|Y_{\alpha}\right\|^{2}=1 / c$ and $\left\|X_{2 \alpha}\right\|^{2}=1 / 4 c$.

Lemma 8.8. For $G$ locally $\mathrm{SU}(2,1)$ one has

$$
\begin{aligned}
\Lambda & =\left\{x X_{\alpha}+y Y_{\alpha}+z X_{2 \alpha} \in \mathfrak{n}\left|2\left(x^{2}+y^{2}\right)+\right| z \mid<1\right\} \\
& =\left\{(Y, Z) \in \mathfrak{n} \mid 2 c\|Y\|^{2}+2 \sqrt{c}\|Z\|<1\right\} .
\end{aligned}
$$

Proof. We want to determine those $Y \in \mathfrak{n}$ which belong to $\Lambda$. By the $M$-invariance of $\Lambda$ we may restrict our attention to elements of the form $Y=x X_{\alpha}+z X_{2 \alpha} \in \Lambda$. We have to find the connected component of those $x, z$ such that

$$
\begin{aligned}
& \left(1+c\left\langle(u+i x) X_{\alpha}+v Y_{\alpha},(u+i x) X_{\alpha}+v Y_{\alpha}\right\rangle\right)^{2} \\
& \quad+4 c\left\langle(w+i z) X_{2 \alpha}+\frac{1}{2} i x v\left[Y_{\alpha}, X_{\alpha}\right],(w+i z) X_{2 \alpha}+\frac{1}{2} i x v\left[Y_{\alpha}, X_{\alpha}\right]\right\rangle=0
\end{aligned}
$$

has no solution for $u, v, w \in \mathbb{R}$. We employ the precedingly collected material on commutators, orthogonality and norms and obtain the equivalent version

$$
\left(1+(u+i x)^{2}+v^{2}\right)^{2}+(w+i(z+2 x v))^{2}=0
$$

for $u, v, w \in \mathbb{R}$. However, this is equivalent to

$$
1+(u+i x)^{2}+v^{2}= \pm i(w+i(z+2 x v))= \pm i w \mp(z+2 x v) .
$$

Comparing real and imaginary parts yields the system of equations

$$
\begin{gathered}
\left(1-x^{2} \pm 2 z\right)+u^{2}+v^{2}=\mp 2 x v, \\
2 u x= \pm w .
\end{gathered}
$$

We can always choose $w$ so that the second equation is satisfied. Hence we look for $x, z$ such that the quadratic equation in $v$

$$
v^{2}-2 x v+\left(1-x^{2} \pm z+u^{2}\right)=0
$$

has no solution for all $u$. Clearly we can take $u=0$ and assume $\pm z=-|z|$. We are left with analyzing the discriminant

$$
4 x^{2}-4\left(1-x^{2}-|z|\right)<0 .
$$

This inequality translates into $2 x^{2}+|z|<1$ and concludes the proof of the lemma.

REMARK 8.9. Consider the domain

$$
\tilde{\Lambda}=\left\{Y \in \mathfrak{n} \mid \exp (i Y) \cdot x_{0} \in \bar{N}_{\mathbb{C}} A_{\mathbb{C}} \cdot x_{0}\right\}_{0} .
$$

It is clear that $\Lambda \subset \tilde{\Lambda}$ and it is easy to determine $\tilde{\Lambda}$ explicitly:

$$
\begin{aligned}
\tilde{\Lambda} & =\left\{(Y, Z) \in \mathfrak{n} \mid\left(1-c\|Y\|^{2}\right)^{2}-4 c\|Z\|^{2}>0\right\} \\
& =\left\{(Y, Z) \in \mathfrak{n} \mid c\|Y\|^{2}+2 \sqrt{c}\|Z\|<1\right\} .
\end{aligned}
$$

Now for $q=0$ we have seen that $\tilde{\Lambda}=\Lambda$. However, as our previous analysis of the $\mathrm{SU}(2,1)$-case shows, one has $\Lambda \neq \tilde{\Lambda}$ in general. 
Before we come to the determination of $\Lambda$ for all rank-one cases some remarks concerning the nature of the constant $c=\frac{1}{4(p+4 q)}$ are appropriate. REMARK 8.10. Let $\mathfrak{g}$ be of real rank one. Let $E \in \mathfrak{g}^{\alpha}$, resp. $E \in \mathfrak{g}^{2 \alpha}$ and set $F=\theta(E), H=[E, F]$. If we assume that $\{H, E, F\}$ is an $\mathfrak{s t}(2)$-triple, i.e. $[H, E]=2 E$ and $[H, F]=-2 F$, then elementary $\mathfrak{s l}(2)$-representation theory gives $\|E\|^{2}=1 / c$ if $E \in \mathfrak{g}^{\alpha}$ and $\|E\|^{2}=1 / 4 c$ if $E \in \mathfrak{g}^{2 \alpha}$.

Theorem 8.11. Let $G$ be a simple Lie group of real rank one.

(i) If $q=0$, then

$$
\Lambda=\left\{Y \in \mathfrak{n} \mid c\|Y\|^{2}<1\right\} .
$$

(ii) If $q>0$, then

$$
\Lambda=\left\{(Y, Z) \in \mathfrak{n} \mid 2 c\|Y\|^{2}+2 \sqrt{c}\|Z\|<1\right\} .
$$

Proof. (i) Lemma 8.7.

(ii) Set

$$
\tilde{\Lambda}=\left\{(Y, Z) \in \mathfrak{n} \mid 2 c\|Y\|^{2}+2 \sqrt{c}\|Z\|<1\right\} .
$$

We first show that $\tilde{\Lambda} \subset \Lambda$. Let $(Y, Z) \in \tilde{\Lambda}, Y \neq 0$ and $Z \neq 0$. Consider the Lie algebra $\mathfrak{g}_{0}$ generated by $Y, \theta(Y), Z, \theta(Z)$. Standard structure theory says that $\mathfrak{g}_{0} \simeq \mathfrak{s u}(2,1)$, see [Hel, Ch.IX, §3]. Choose $E_{\alpha} \in \mathbb{R} Y$ and $E_{2 \alpha} \in \mathbb{R} Z$ such that $\left\{\left[E_{\alpha}, \theta\left(E_{\alpha}\right)\right], E_{\alpha}, \theta\left(E_{\alpha}\right)\right\}$ as well as $\left\{\left[E_{2 \alpha}, \theta\left(E_{2 \alpha}\right)\right]\right.$, $\left.E_{2 \alpha}, \theta\left(E_{2 \alpha}\right)\right\}$ are $\mathfrak{s l}(2)$-triples. Let $y, z \in \mathbb{R}$ such that $Y=y E_{\alpha}$ and $Z=z E_{2 \alpha}$. In view of Remark 8.10, the condition $2 c\|Y\|^{2}+2 \sqrt{c}\|Z\|<1$ is equivalent to $2 y^{2}+|z|<1$. But this is just the condition for $\exp (i(Y+Z)) \cdot x_{0}$ to be contained in the crown domain $\Xi_{0}$ for the group $G_{0}=\left\langle\exp \mathfrak{g}_{0}\right\rangle<G$ (see Lemma 8.8). Now, with the obvious notation, we have $\Omega=\Omega_{0}$ and so $\Xi_{0} \subset \Xi$. This concludes the proof of $\tilde{\Lambda} \subset \Lambda$.

It remains to verify that $\Lambda \subset \tilde{\Lambda}$. For that it is sufficient to show the following: if $(Y, Z) \in \partial \tilde{\Lambda}$, then $(Y, Z) \notin \Lambda$. Let also $(Y, Z) \in \partial \tilde{\Lambda}$ with $(Y, Z) \in \Lambda$ and let $\mathfrak{g}_{0}$ as before. Note that $\exp (i(Y+Z)) \cdot x_{0} \in \partial \Xi_{0}$. As $\Xi_{0} \subset \Xi$ is closed and $\exp (i(Y+Z)) \cdot x_{0} \in \Xi$, there would exist $G_{0}$-domain $\Xi_{0}^{\prime} \subset X_{0, \mathbb{C}}$, properly containing $\Xi_{0}$, and on which $G_{0}$ acts properly. But this contradicts Theorem 4.1.

8.2 The case where $\Xi$ is a Hermitian symmetric space. It can happen that $\Xi$ allows additional symmetries, i.e. the group of holomorphic automorphisms is strictly larger then $G$. For example, when $X=G / K$ is a Hermitian symmetric space, then $\Xi$ is biholomorphic to $X \times \bar{X}$ where $\bar{X}$ denotes $X$ endowed with the opposite complex structure (see $[\mathrm{KrS} 2$, Th. 7.7]). In this example $\Xi=X \times \bar{X}$ is again a Hermitian symmetric space and $\operatorname{Aut}(\Xi)=G \times G$ is twice the size of $G=\operatorname{Aut}(X)$. In [KrS2, Th. 7.8], one 
can find a classification of all those cases where $\Xi$ is a Hermitian symmetric space for a larger group $S$. For all these cases it turns out that there is an interesting subset $\Lambda^{+} \subset \Lambda$ such that $\Xi=G \exp \left(i \Lambda^{+}\right) \cdot x_{0}$, and moreover, it is possible to give a precise relation between unipotent $G$-orbits through $\exp \left(i \Lambda^{+}\right) \cdot x_{0}$ and the elliptic $G$-orbits through $\exp (i \Omega) \cdot x_{0}$. As the case of the symplectic group is of special interest, in particular for later applications to automorphic forms, and as it is always good to have a illustrating example, we shall begin with a discussion for this group.

8.2.1 The symplectic group. In this section $G=\operatorname{Sp}(n, \mathbb{R})$ for $n \geq 1$. Let us denote by $\operatorname{Sym}(n, \mathbb{R})$, resp. $M_{+}(n, \mathbb{R})$, the symmetric, resp. strictly upper triangular, matrices in $M(n, \mathbb{R})$. Our choices of $\mathfrak{a}$ and $\mathfrak{n}$ shall be

$$
\mathfrak{a}=\left\{\operatorname{diag}\left(t_{1}, \ldots, t_{n},-t_{1}, \ldots,-t_{n}\right) \mid t_{i} \in \mathbb{R}\right\}
$$

and

$$
\mathfrak{n}=\left\{\left(\begin{array}{cc}
Y & Z \\
0 & -Y^{T}
\end{array}\right) \mid Z \in \operatorname{Sym}(n, \mathbb{R}), Y \in M_{+}(n, \mathbb{R})\right\} .
$$

Of special interest is an abelian subalgebra of $\mathfrak{n}$

$$
\mathfrak{n}^{+}=\left\{\left(\begin{array}{cc}
0 & Z \\
0 & 0
\end{array}\right) \mid Z \in \operatorname{Sym}(n, \mathbb{R})\right\} .
$$

We recall that the maximal compact subgroup $K<G$ is isomorphic to $U(n)$ and that $X=G / K$ admits a natural realization as a Siegel upper halfplane:

$$
X=\operatorname{Sym}(n, \mathbb{R})+i \operatorname{Sym}^{+}(n, \mathbb{R}) \subset \operatorname{Sym}(n, \mathbb{C})
$$

where $\operatorname{Sym}^{+}(n, \mathbb{R})$ denotes the positive definite symmetric matrices. The action of $G$ on $X$ is given by generalized fractional transformations: if $g=\left(\begin{array}{cc}A & B \\ C & D\end{array}\right) \in G$ and $Z \in X$, then

$$
g(Z)=(A Z+B)(C Z+D)^{-1} .
$$

Notice that the base point $x_{0}$ with stabilizer $K$ becomes $x_{0}=i I_{n}$ with $I_{n}$ the identity matrix. The natural realization of $\bar{X}$ is the lower half plane

$$
\bar{X}=\operatorname{Sym}(n, \mathbb{R})-i \operatorname{Sym}^{+}(n, \mathbb{R}) .
$$

In the sequel we view $X$ inside of $X \times \bar{X}$ as a totally real submanifold via the embedding

$$
X \hookrightarrow X \times \bar{X}, \quad Z \mapsto(Z, \bar{Z})
$$

where $\bar{Z}$ denotes the complex conjugation in $\operatorname{Sym}(n, \mathbb{C})$ with respect to the real form $\operatorname{Sym}(n, \mathbb{R})$. As we remarked earlier, $\Xi$ is naturally biholomorphic to $X \times \bar{X}$. Let us now consider a domain in $\mathfrak{n}^{+}$

$$
\Lambda^{+}=\left\{Y \in \mathfrak{n}^{+} \mid \exp (i Y) \cdot x_{0} \in \Xi\right\}_{0} .
$$


Theorem 8.12. For $G=\operatorname{Sp}(n, \mathbb{R})$ the following assertions hold:

(i) If $\|\cdot\|$ denotes the operator norm on $M(n, \mathbb{R})$, then

$$
\Lambda^{+}=\left\{\left(\begin{array}{cc}
0 & Z \\
0 & 0
\end{array}\right) \in \mathfrak{n}^{+} \mid\|Z\|<1\right\} .
$$

(ii) With $\Lambda^{++}=\Lambda \cap \operatorname{diag}(n, \mathbb{R})$ one has

(a) $\Lambda^{++}=\left\{\operatorname{diag}\left(t_{1}, \ldots, t_{n}\right) \in \mathfrak{n}^{+}|| t_{i} \mid<1\right\}$

(b) $\Lambda^{+}=\operatorname{Ad} K_{0}\left(\Lambda^{++}\right)$with $K_{0}=\mathrm{SO}(n, \mathbb{R})<K$.

(iii) $\Xi=G \exp \left(i \Lambda^{+}\right) \cdot x_{0}=G \exp \left(i \Lambda^{++}\right) \cdot x_{0}$.

Proof. (i) Let $Z \in \operatorname{Sym}(n, \mathbb{R})$ and $\tilde{Z}=\left(\begin{array}{ll}0 & Z \\ 0 & 0\end{array}\right)$ the corresponding element in $\mathfrak{n}^{+}$. Then

and accordingly

$$
\exp (i \tilde{Z})=\left(\begin{array}{cc}
I_{n} & i Z \\
0 & I_{n}
\end{array}\right)
$$

$$
\exp (i \tilde{Z}) \cdot x_{0}=\exp (i \tilde{Z})\left(i I_{n},-i I_{n}\right)=\left(i\left(I_{n}+Z\right),-i\left(I_{n}-Z\right)\right) .
$$

Therefore $\exp (i \tilde{Z}) \cdot x_{0} \in X \times \bar{X}$ if and only if $I_{n}+Z \in \operatorname{Sym}^{+}(n, \mathbb{R})$ and $I_{n}-Z \in \operatorname{Sym}^{+}(n, \mathbb{R})$. Clearly, this is equivalent to $\|Z\|<1$ and the proof of (i) is finished.

(ii) This is immediate from (i).

(iii) It is enough to show that $\Xi=G \exp \left(i \Lambda^{++}\right) \cdot x_{0}$ and for that it suffices to verify that $\exp (i \Omega) \cdot x_{0} \subset G \exp \left(i \Lambda^{++}\right) \cdot x_{0}$. Now, as $\Sigma$ is of type $C_{n}$, the domain $\Omega$ is a cube

$$
\Omega=\left\{\operatorname{diag}\left(t_{1}, \ldots, t_{n},-t_{1}, \ldots,-t_{n}\right)|| t_{i} \mid<\frac{\pi}{4}\right\} .
$$

Let us write $E_{i j}$ for the elementary matrices in $M(2 n, \mathbb{R})$. Then for each $1 \leq j \leq n$ we define an $\mathfrak{s l}(2)$-subalgebra $\mathfrak{g}_{j}$ by

$$
\mathfrak{g}_{j}=\operatorname{span}_{\mathbb{R}}\left\{E_{j j}-E_{j+n, j+n}, E_{j, j+n}, E_{n+j, j}\right\} .
$$

We note that the $\mathfrak{g}_{j}$ pairwise commute and so $\mathfrak{g}_{0}=\mathfrak{g}_{1} \oplus \ldots \oplus \mathfrak{g}_{n}$ is a subalgebra of $\mathfrak{g}$ which contains $\mathfrak{a}$. Now we are in the situation to use $\mathfrak{s l}(2)$ reduction and the assertion becomes a consequence of Lemma 3.3.

For later reference we wish to make the last part of the above theorem more precise. For $\mathbf{z}=\left(z_{1}, \ldots, z_{n}\right) \in \mathbb{C}^{n}$ let us define a matrix in $N_{\mathbb{C}}+$

$$
n_{\mathbf{z}}=\left(\begin{array}{cc}
I_{n} & \operatorname{diag}(\mathbf{z}) \\
0 & I_{n}
\end{array}\right) .
$$

Moreover if $\mathbf{z} \in\left(\mathbb{C}^{*}\right)^{n}$, then we set

$$
a_{\mathbf{z}}=\operatorname{diag}\left(z_{1}, \ldots, z_{n}, z_{1}^{-1}, \ldots, z_{n}^{-1}\right) \in A_{\mathbb{C}} .
$$

In the course of the proof of Theorem 8.12 (iii) we have shown the following: 
Lemma 8.13. Let $\mathbf{t}=\left(t_{1}, \ldots, t_{n}\right) \in \mathbb{R}^{n}$ with $\left|t_{i}\right|<\pi / 4$. Set $\mathbf{e}^{i \mathbf{t}}=$ $\left(e^{i t_{1}}, \ldots, e^{i t_{n}}\right)$ and $\sin (2 \mathbf{t})=\left(\sin 2 t_{1}, \ldots, \sin 2 t_{n}\right)$. Then

$$
G n_{\sin (2 \mathbf{t})} \cdot x_{0}=G a_{\mathbf{e}^{i t}} \cdot x_{0} .
$$

8.2.2 The general case of Hermitian $\boldsymbol{\Xi}$. In this subsection we will assume that $\Xi$ is a Hermitian symmetric space for an overgroup $S \supset G$. From a technical point of view it is however better to work with an alternative characterization, namely (cf. [KrS2, Th. 7.8]) $\Sigma$ is of type $C_{n}$ or $B C_{n}$ for $n \geq 2$ or $\mathfrak{g}=\mathfrak{s o}(1, k)$ with $k \geq 2$ for the rank one cases.

If $\Sigma$ is of type $C_{n}$ or $B C_{n}$, then

$$
\Sigma=\left\{ \pm \gamma_{i} \pm \gamma_{j} \mid 1 \leq i, j \leq n\right\} \backslash\{0\} \cup\left\{ \pm \frac{1}{2} \gamma_{i}: 1 \leq i \leq n\right\}
$$

with the second set on the right to be considered not present in the $C_{n^{-}}$-case. As a positive system of $\Sigma$ we choose

$$
\Sigma^{+}=\left\{\gamma_{i} \pm \gamma_{j} \mid 1 \leq i \leq j \leq n\right\} \backslash\{0\} \cup\left\{\frac{1}{2} \gamma_{i}: 1 \leq i \leq n\right\} .
$$

Further we consider the $A_{n-1}$-subsystem

$$
\Sigma_{0}=\left\{ \pm \gamma_{i} \mp \gamma_{j} \mid 1 \leq i \neq j \leq n\right\}
$$

and set

$$
\Sigma^{++}=\Sigma^{+} \cap\left(\Sigma^{+} \backslash \Sigma_{0}\right) \text { and } \Sigma^{--}=-\Sigma^{++} .
$$

Next we define subalgebras of $\mathfrak{g}$ by

$$
\mathfrak{n}^{+}=\bigoplus_{\alpha \in \Sigma^{++}} \mathfrak{g}^{\alpha}, \quad \mathfrak{n}^{-}=\bigoplus_{\alpha \in \Sigma^{--}} \mathfrak{g}^{\alpha} \quad \text { and } \quad \mathfrak{g}(0)=\mathfrak{a} \oplus \mathfrak{m} \oplus \bigoplus_{\alpha \in \Sigma_{0}} \mathfrak{g}^{\alpha} .
$$

We note that $\mathfrak{n}^{+}$is a subalgebra of $\mathfrak{n}$ and that

$$
\mathfrak{g}=\mathfrak{n}^{-} \oplus \mathfrak{g}(0) \oplus \mathfrak{n}^{+}
$$

is a direct decomposition with $\left[\mathfrak{g}(0), \mathfrak{n}^{ \pm}\right] \subset \mathfrak{n}^{ \pm}$. Define elements $T_{j} \in \mathfrak{a}$ by the requirement $\gamma_{i}\left(T_{j}\right)=\delta_{i j}$ and note that

$$
\left.\Omega=\bigoplus_{j=1}^{n}\right]-\frac{\pi}{4}, \frac{\pi}{4}\left[T_{j}\right.
$$

Now for an element $Y_{j} \in \mathfrak{g}^{2 \gamma_{j}}$ we find $E_{j} \in \mathbb{R} Y_{j}$, unique up to sign, such that $\left\{T_{j}, E_{j}, \theta\left(E_{j}\right)\right\}$ form an $\mathfrak{s l}(2)$-triple. Define $y_{j} \in \mathbb{R}$ by $Y_{j}=y_{j} E_{j}$. With that we can define an open ball in $\bigoplus_{j=1}^{n} \mathfrak{g}^{2 \gamma_{j}}$ by

$$
\Lambda^{++}=\left\{Y=\sum_{j=1}^{n} Y_{j} \in \bigoplus_{j=1}^{n} \mathfrak{g}^{2 \gamma_{j}}|| y_{j} \mid<1\right\}
$$

Further write $\mathfrak{k}(0)=\mathfrak{g}(0) \cap \mathfrak{k}$ and set $K(0)=\exp \mathfrak{k}(0)$. Finally we define a subset of $\mathfrak{n}^{+}$by

$$
\Lambda^{+}=\operatorname{Ad} K_{0}\left(\Lambda^{++}\right)
$$


REMARK 8.14. Define an abelian subspace of $\mathfrak{n}^{+}$by $\mathfrak{n}^{++}=\bigoplus_{\alpha \in \Sigma^{++} \cap C_{n}} \mathfrak{g}^{\alpha}$. Then $\Lambda^{+}$is a bounded convex domain in $\mathfrak{n}^{++}$.

Theorem 8.15. If $\Xi$ is a Hermitian symmetric space for an overgroup $S \supset G$, then the following assertions hold:

(i) If $T=\sum_{j=1}^{n} t_{j} T_{j} \in \Omega$, and $\left\{T_{j}, E_{j}, \theta\left(E_{j}\right)\right\}$ is any $\mathfrak{s l}(2)$-triple with $E_{j} \in \mathfrak{g}^{2 \gamma_{j}}$, then

$$
G \exp \left(i \sum_{j=1}^{n} \sin \left(2 t_{j}\right) E_{j}\right) \cdot x_{0}=G \exp \left(i \sum_{j=1}^{n} t_{j} T_{j}\right) \cdot x_{0} .
$$

(ii) $\Xi=G \exp \left(i \Lambda^{+}\right) \cdot x_{0}=G \exp \left(i \Lambda^{++}\right) \cdot x_{0}$.

Proof. (i) We define subalgebras of $\mathfrak{g}$ which are isomorphic to $\mathfrak{s l}(2, \mathbb{R})$ by $\mathfrak{g}_{j}=\mathbb{R} T_{j} \oplus \mathbb{R} E_{j} \oplus \mathbb{R} \theta\left(E_{j}\right)$. The $\mathfrak{g}_{j}$ 's commute in $\mathfrak{g}$ and so $\mathfrak{g}_{0}=\mathfrak{g}_{1} \oplus \ldots \oplus \mathfrak{g}_{n}$ defines a subalgebra of $\mathfrak{g}$. In view of Lemma 3.3, the assertion now follows by $\mathfrak{s l}(2)$-reduction.

(ii) This is a consequence of (i).

REMARK 8.16. If $\mathfrak{g}$ is Hermitian and of tube type, then $\Lambda^{+}$is a bounded open convex set in $\mathfrak{n}^{+}$and

$$
\Lambda^{+}=\left\{Z \in \mathfrak{n}^{+} \mid \exp (i Z) \cdot x_{0} \in \Xi\right\}_{0} .
$$

This can be proved as in the $\operatorname{Sp}(n)$-case by employing the machinery of Jordan algebras.

8.3 Some partial results for the special linear groups. In this subsection we exclusively deal with $G=\operatorname{Sl}(n, \mathbb{R})$. To determine the exact shape of $\Lambda$ for $n \geq 3$ seems to be very challenging; already the case of $n=3$ appears to be very intricate. Instead we will exhibit a fairly large cubedomain inside of $\Lambda$; further we will estimate the corresponding hyperbolic parameterization.

In order to perform reasonably efficient computations we use the matrix model for $X_{\mathbb{C}}$. Let us denote by $\operatorname{Sym}(n, \mathbb{C})_{\operatorname{det}=1}$ the affine variety of complex symmetric matrices with unit determinant. The map

$$
X_{\mathbb{C}}=\operatorname{Sl}(n, \mathbb{C}) / \operatorname{SO}(n, \mathbb{C}) \rightarrow \operatorname{Sym}(n, \mathbb{C})_{\operatorname{det}=1}, \quad g K_{\mathbb{C}} \mapsto g g^{t}
$$

is an isomorphism. Within this model for $X_{\mathbb{C}}$, the Riemannian symmetric space $X$ identifies with $\operatorname{Sym}(n, \mathbb{R})_{\operatorname{det}=1}^{+}$, the determinant one section in the cone of positive definite symmetric matrices. Now the crown domain $\Xi$ contains the determinant one cut $\Xi_{0}$ of the tube domain, i.e.

$$
\Xi_{0}=\left\{Z \in X_{\mathbb{C}} \mid \operatorname{Re} Z \gg 0\right\}
$$


As usual we write $E_{i j}=\left(\delta_{k i} \delta_{i j}\right)_{l j}$ for the elementary matrices. We choose $N$ to be the group of unipotent upper triangular matrices and consider the mapping

$m: \mathbb{C}^{n-1} \rightarrow N_{\mathbb{C}}, \quad\left(z_{1}, \ldots, z_{n-1}\right) \mapsto \exp \left(z_{1} E_{12}\right) \cdot \ldots \cdot \exp \left(z_{n-1} E_{n-1 n}\right)$.

In matrix notation $m$ is given by

$$
m\left(z_{1}, \ldots, z_{n-1}\right)=\left(\begin{array}{ccccc}
1 & z_{1} & & & \\
& 1 & z_{2} & & \\
& & \ddots & \ddots & \\
& & & 1 & z_{n-1} \\
& & & & 1
\end{array}\right) .
$$

We define a subset of $N_{\mathbb{C}}$ by

and claim

$$
\mathcal{N}^{+}=m\left(i \prod_{j=1}^{n-1}(-1,1)\right),
$$

Proposition 8.17. $\mathcal{N}^{+} \cdot x_{0} \subset \Xi_{0}$. In particular $G \mathcal{N}^{+} \cdot x_{0} \subset \Xi_{0} \subset \Xi$.

Proof. This is an elementary matrix computation. Let $\left(t_{1}, \ldots, t_{n-1}\right) \in \mathbb{R}^{n-1}$, $\left|t_{i}\right|<1$ and set

$$
n=\left(\begin{array}{ccccc}
1 & i t_{1} & & & \\
& 1 & i t_{2} & & \\
& & \ddots & \ddots & \\
& & & 1 & i t_{n-1} \\
& & & & 1
\end{array}\right) .
$$

One has to verify that $\operatorname{Re}\left(n n^{t}\right) \gg 0$. A straightforward calculation yields

Therefore

$$
n n^{t}=\left(\begin{array}{ccccc}
1 & i t_{1} & & & \\
i t_{1} & 1-t_{1}^{2} & i t_{2} & & \\
& i t_{2} & \ddots & \ddots & \\
& & \ddots & 1-t_{n-2}^{2} & i t_{n-1} \\
& & & i t_{n-1} & 1-t_{n-1}^{2}
\end{array}\right) .
$$

$$
\operatorname{Re}\left(n n^{t}\right)=\left(\begin{array}{cccc}
1 & & & \\
& 1-t_{1}^{2} & & \\
& & \ddots & \\
& & & 1-t_{n-1}^{2}
\end{array}\right) \gg 0 .
$$

Next we discuss hyperbolic parameterization for elements in $\mathcal{N}^{+}$. Here our results are somewhat partial but perhaps still interesting. For what 
follows we are indebted to Philip Foth. For $t \in \mathbb{R}$ with $|t|<1$ we consider the element

$$
z(t)=m(i(t, \ldots, t))=\left(\begin{array}{ccccc}
1 & i t & & & \\
& 1 & i t & & \\
& & \ddots & \ddots & \\
& & & 1 & i t \\
& & & & 1
\end{array}\right) .
$$

We wish to estimate the element $a(t) \in \exp (i \pi \Omega / 2)$ for which

$$
G z(t) \cdot x_{0}=G a(t) \cdot x_{0}
$$

holds. The result is as follows.

Proposition 8.18. Let $G=\operatorname{Sl}(n, \mathbb{R})$. Fix $t \in \mathbb{R},|t|<1$ and set $z(t)=m(i(t, \ldots, t))$. Then $G z(t) \cdot x_{0}=G a(t) \cdot x_{0}$ with

$$
a(t)=\operatorname{diag}\left(e^{i \phi_{1}(t)}, \ldots, e^{i \phi_{n}(t)}\right), \quad \operatorname{diag}\left(\phi_{1}(t), \ldots, \phi_{n}(t)\right) \in \pi \Omega / 2
$$

and

$$
\left|\phi_{j}(t)\right| \leq\left|\frac{1}{2} \tan ^{-1}\left(\frac{2 t}{1-t^{2}}\right)\right| \quad(1 \leq j \leq n) .
$$

Proof. We proceed indirectly and use the complex convexity Theorem 5.4. For $k \in K$ we have to show that the components of $\operatorname{Im} \log a_{\mathbb{C}}(k z(t))$ satisfy the estimate (8.4). To compute $a_{\mathbb{C}}(k z(t))$ we write out the corresponding matrix identity:

$$
\begin{aligned}
& \underbrace{\left(\begin{array}{ccccc}
1 & * & \ldots & \ldots & * \\
& 1 & * & \ldots & * \\
& & \ddots & \ddots & \vdots \\
& & & 1 & * \\
& & & & 1
\end{array}\right)}_{\in N_{\mathbb{C}}} \cdot \underbrace{\left(\begin{array}{cccc}
* & \ldots & * \\
* & \ldots & * \\
\vdots & \vdots & \vdots \\
* & \ldots & * \\
k_{1} & \ldots & k_{n}
\end{array}\right)}_{\in K} \cdot \underbrace{\left(\begin{array}{ccccc}
1 & i t & & & \\
& 1 & i t & & \\
& & \ddots & \ddots & \\
& & & 1 & i t \\
& & & & 1
\end{array}\right)}_{=z(t)} \\
& =\underbrace{\left(\begin{array}{cccc}
a_{\mathbb{C}, 1}(t) & & & \\
& a_{\mathbb{C}, 2}(t) & & \\
& & \ddots & \\
& & & a_{\mathbb{C}, n}(t)
\end{array}\right)}_{\in A \exp (i \pi \Omega / 2)} \cdot \underbrace{\left(\begin{array}{ccc}
* & \ldots & * \\
* & \ldots & * \\
\vdots & \vdots & \vdots \\
k_{1}^{\prime} & \ldots & k_{n}^{\prime}
\end{array}\right)}_{\in K_{\mathbb{C}}} .
\end{aligned}
$$

We match the bottom rows and arrive at

$$
\left(k_{1}, i t k_{1}+k_{2}, i t k_{2}+k_{3}, \ldots, i t k_{n_{1}}+k_{n}\right)=a_{\mathbb{C}, n}(t)\left(k_{1}^{\prime}, \ldots, k_{n}^{\prime}\right) .
$$

We square the entries and sum them up:

$$
1-t^{2}\left(k_{1}^{2}+\ldots+k_{n-1}^{2}\right)+2 i t\left(k_{1} k_{2}+\ldots+k_{n-1} k_{n}\right)=a_{\mathbb{C}, n}(t)^{2} .
$$


The result is

$$
\left|\phi_{n}(t)\right|=\left|\frac{1}{2} \tan ^{-1}\left(\frac{2 t\left(k_{1} k_{2}+\ldots+k_{n-1} k_{n}\right)}{1-t^{2}\left(k_{1}^{2}+\ldots+k_{n-1}^{2}\right)}\right)\right| .
$$

Finally we use the estimates $k_{1}^{2}+\ldots+k_{n-1}^{2} \leq 1$ and $k_{1} k_{2}+\ldots+k_{n-1} k_{n} \leq 1$ and obtain that

$$
\left|\phi_{n}(t)\right|=\left|\frac{1}{2} \tan ^{-1}\left(\frac{2 t}{1-t^{2}}\right)\right| .
$$

This proves (8.4) for the last entry. The general case follows by Weyl group invariance.

REMARK 8.19. One can show that $z(t) \cdot x_{0} \in \Xi$ precisely for $|t|<1$.

\section{Exponential Decay of Maaß Cusp Forms I: The Example of $G=\operatorname{Sl}(2, \mathbb{R})$}

It is a result obtained by Langlands that cuspidal automorphic forms are of rapid decay. But actually more is true and the decay is of exponential type. The purpose of this section is to give an introduction to this circle of problems with a solid discussion of the case of $G=\operatorname{Sl}(2, \mathbb{R})$. We will restrict our attention to Maaß cusp forms and to the modular group $\Gamma=\operatorname{Sl}(2, \mathbb{Z})$ in order to keep the exposition basic. It is possible to verify the exponential decay by our explicit knowledge of the Whittaker functions in this case. This will be presented first. In general however, concrete knowledge of the Whittaker functions is not available and an alternative approach is needed. It was Joseph Bernstein who came up with the idea to use analytic continuation to obtain exponential decay. We shall present his ideas in the geometric framework which we developed in the preceding section.

9.1 Concrete approach. For the rest of this section we let $G=\operatorname{Sl}(2, \mathbb{R})$ and keep our choices including notation from section 3 . In the sequel we will identify $X=G / K$ with the upper half plane, i.e. $X=\{z \in \mathbb{C} \mid \operatorname{Im} z>0\}$ and $G$ acting by fractional linear transformations:

$$
g(z)=\frac{a z+b}{c z+d} \quad \text { for } g=\left(\begin{array}{ll}
a & b \\
c & d
\end{array}\right) \in G, z \in X .
$$

In these coordinates the base point $x_{0}$ is the imaginary unit $x_{0}=i$ and the Iwasawa decomposition states that the map

$$
N \times A \rightarrow X, \quad\left(n_{x}, a_{y}\right) \mapsto n_{x} a_{y}(i)=x+i y,
$$

where

$$
n_{x}=\left(\begin{array}{cc}
1 & x \\
0 & 1
\end{array}\right) \in N \quad \text { and } \quad a_{y}=\left(\begin{array}{cc}
\sqrt{y} & 0 \\
0 & \frac{1}{\sqrt{y}}
\end{array}\right) \in A
$$


with $x \in \mathbb{R}, y>0$, is a diffeomorphism. The Laplace-Beltrami operator of $X$ is given by $\Delta=-y^{2}\left(\partial_{x}^{2}+\partial_{y}^{2}\right)$ and we note that $\mathbb{D}(X)=\mathbb{C}[\Delta]$. We make a simpler choice for a lattice $\Gamma<G$, namely $\Gamma=\operatorname{Sl}(2, \mathbb{Z})$ the modular group. Then by a Maaß automorphic form we understand an analytic function $\phi: X \rightarrow \mathbb{C}$ such that

- $\phi$ is $\Gamma$-invariant;

- $\phi$ is an eigenfunction for $\mathbb{D}(X)$, i.e. there exists $\lambda \in \mathbb{C}$ such that $\Delta \phi=\lambda(1-\lambda) \phi$

- $\phi$ is of moderate growth, i.e. there exists $\alpha \in \mathbb{R}$ such that

$$
|\phi(x+i y)| \ll y^{\alpha} \quad(y>1) .
$$

Moreover, a Maaß automorphic form is called a cusp form if

$$
\int_{N \cap \Gamma \backslash N} \phi(n z) d n=0 \text { for all } z \in X .
$$

Note that $N \cap \Gamma=\left(\begin{array}{ll}1 & \mathbb{Z} \\ 0 & 1\end{array}\right)$ so that $N \cap \Gamma \backslash N \simeq \mathbb{Z} \backslash \mathbb{R}$ is a circle. In our special case the results of Langlands reads as follows.

Theorem 9.1. Maaß cusp forms $\phi$ are of rapid decay, i.e.

for any $\alpha \in \mathbb{R}$.

$$
|\phi(x+i y)| \ll y^{\alpha} \quad(y>1)
$$

However, more is true and we can state

Theorem 9.2. Maaß cusp forms $\phi$ are of exponential decay, i.e. there is a constant $C>0$ such that

$$
|\phi(x+i y)| \leq C e^{-2 \pi y} \quad(y>1) .
$$

Before we prove this theorem, we will recall the Whittaker expansion of a Maaß cusp form: If $\phi$ is a Maaß cusp form with $\Delta \phi=\lambda(1-\lambda) \phi$, then

$$
\phi(x+i y)=\sum_{n \in \mathbb{Z}^{x}} a_{n} \sqrt{y} K_{\nu}(2 \pi|n| y) e^{2 \pi i n x},
$$

where $K_{\nu}$ is the McDonald Bessel function

$$
K_{\nu}(y)=\frac{1}{2} \int_{0}^{\infty} e^{-y\left(t+\frac{1}{t}\right) / 2} t^{\nu} \frac{d t}{t} \quad(y>0)
$$

with parameter $\lambda=\frac{1}{2}+\nu$ and the $a_{n}$ are complex numbers satisfying the Hecke bound

$$
\left|a_{n}\right| \ll|n|^{1 / 2} .
$$

As a final piece of information we need the asymptotic expansion of the Bessel function

$$
K_{\nu}(y) \sim\left(\frac{\pi}{2 y}\right)^{1 / 2} e^{-y} \cos (\nu \pi) .
$$

We can now prove Theorem 9.2. 
Proof. We plug the estimates (9.2) and (9.3) into the Fourier expansion (9.1) and use the convention that $C$ denotes a positive constant whose actual value may change from line to line: for $y$ large we obtain

$$
\begin{aligned}
|\phi(x+i y)| & \leq \sum_{n \neq 0}\left|a_{n}\right| \sqrt{y} \cdot\left|K_{\nu}(2 \pi|n| y)\right| \\
& \leq C \sum_{n \neq 0}|n|^{\frac{1}{2}} \sqrt{y}\left(\frac{\pi}{2 \pi|n| y}\right)^{1 / 2} e^{-2 \pi|n| y} \\
& \leq C \sum_{n \neq 0} e^{-2 \pi|n| y} \\
& \leq C \frac{e^{-2 \pi y}}{1-e^{-2 \pi y}} \\
& \leq C e^{-2 \pi y} .
\end{aligned}
$$

9.2 The method of analytic continuation. We now present an alternative approach to Theorem 9.2, essentially due to Bernstein, which uses the method of analytic continuation. The final result is slightly weaker than the optimal estimate in Theorem 9.2, but this will be balanced by the conceptionality of the approach.

Let $\phi$ be a Maaß cusp form. Let us fix $y>0$ and consider the 1-periodic function

$$
F_{y}: \mathbb{R} \rightarrow \mathbb{C}, \quad u \mapsto \phi\left(n_{u} a_{y}(i)\right)=\phi(u+i y) .
$$

This function being smooth and periodic admits a Fourier expansion

$$
F_{y}(u)=\sum_{n \neq 0} A_{n}(y) e^{2 \pi i n x} .
$$

Here, $A_{n}(y)$ are complex numbers depending on $y$. Now observe that

and so

$$
n_{u} a_{y}=a_{y} a_{y}^{-1} n_{u} a_{y}=a_{y} n_{u / y}
$$

$$
F_{y}(u)=\phi\left(a_{y} n_{u / y} \cdot x_{0}\right) \text {. }
$$

As $\phi$ is a $\mathbb{D}(X)$-eigenfunction, it admits a holomorphic continuation to $\Xi$ and thus it follows from Lemma 3.3 and Theorem 3.4 that $F_{y}$ admits a holomorphic continuation to the strip domain

$$
S_{y}=\{w=u+i v \in \mathbb{C}|| v \mid<y\} .
$$

Now, let $\epsilon>0, \epsilon$ small. Then, for $n>0$, we proceed with Cauchy

$$
\begin{aligned}
A_{n}(y) & =\int_{0}^{1} F_{y}(u-i(1-\epsilon) y) e^{-2 \pi i n(u-i(1-\epsilon) y)} d u \\
& =e^{-2 \pi n(1-\epsilon) y} \int_{0}^{1} F_{y}(u-i(1-\epsilon) y) e^{-2 \pi i n u} d u
\end{aligned}
$$




$$
=e^{-2 \pi n(1-\epsilon) y} \int_{0}^{1} \phi\left(a_{y} n_{u / y} n_{-i(1-\epsilon)} \cdot x_{0}\right) e^{-2 \pi i n u} d u .
$$

Thus we get, for all $\epsilon>0$ and $n \neq 0$ the inequality

$$
\left|A_{n}(y)\right| \leq e^{-2 \pi|n| y(1-\epsilon)} \sup _{\Gamma g \in \Gamma \backslash G}\left|\phi\left(\Gamma g n_{ \pm i(1-\epsilon)} \cdot x_{0}\right)\right| .
$$

We need an estimate.

Lemma 9.3. Let $\phi$ be a Maaß cusp form. Then there exists a constant $C$ only depending on $\lambda$ such that for all $0<\epsilon<1$

$$
\sup _{\Gamma g \in \Gamma \backslash G}\left|\phi\left(\Gamma g n_{i(1-\epsilon)} \cdot x_{0}\right)\right| \leq C|\log \epsilon|^{1 / 2} .
$$

Proof. Let $-\pi / 4<t_{\epsilon}<\pi / 4$ be such that $\pm(1-\epsilon)=\sin 2 t_{\epsilon}$. Then, by Lemma 3.3 we have $G n_{ \pm i(1-\epsilon)} \cdot x_{0}=G a_{\epsilon} \cdot x_{0}$ with $a_{\epsilon}=\left(\begin{array}{cc}e^{i \epsilon_{\epsilon}} & 0 \\ 0 & e^{-i t_{\epsilon}}\end{array}\right)$. Now note that $t_{\epsilon} \approx \pi / 4-\sqrt{2 \epsilon}$ and thus [KrS1, Th. $5.1 \&$ Th. 6.17], give that

$$
\sup _{\Gamma g \in \Gamma \backslash G}\left|\phi\left(g a_{\epsilon} \cdot x_{0}\right)\right| \leq C|\log \epsilon|^{1 / 2} .
$$

This concludes the proof of the lemma.

We use the estimates in Lemma 9.3 in (9.4) and get

$$
\left|A_{n}(y)\right| \leq C e^{-2 \pi|n| y(1-\epsilon)}|\log \epsilon|^{1 / 2}
$$

and specializing to $\epsilon=1 / y$ gives that

$$
\left|A_{n}(y)\right| \leq C e^{-2 \pi|n|(y-1)}(\log y)^{1 / 2} .
$$

This in turn yields for $y>2$ that

$$
\begin{aligned}
|\phi(i y)| & =\left|F_{y}(0)\right| \leq \sum_{n \neq 0}\left|A_{n}(y)\right| \\
& \leq C(\log y)^{1 / 2} \sum_{n \neq 0} e^{-2 \pi|n|(y-1)} \\
& \leq C(\log y)^{1 / 2} \cdot e^{-2 \pi y} .
\end{aligned}
$$

It is clear, that we can replace $F_{y}$ by $F_{y}(\cdot+x)$ for any $x \in \mathbb{R}$ without altering the estimate. Thus we have proved:

Theorem 9.4. Let $\phi$ be a Maaß cusp form. Then there exists a constant $C>0$, only depending on $\lambda$, such that

$$
|\phi(x+i y)| \leq C(\log y)^{1 / 2} \cdot e^{-2 \pi y} \quad(y>2) .
$$

REMARK 9.5. It is not too hard to make the constant in the theorem precise. We will do this in the next section when we give a general discussion of the rank-one cases. 


\section{Exponential Decay of Maaß Cusp Forms II: The Rank-One Cases}

The example of $G=\operatorname{Sl}(2, \mathbb{R})$ admits a straightforward generalization to all rank-one cases and this will be outlined below. Throughout this section we let $G$ be of real rank one, i.e. $\operatorname{dim} \mathfrak{a}=1$. We fix a noncocompact lattice $\Gamma<G$ and call a parabolic subgroup $M A N$ cuspidal for $\Gamma$ if $\Gamma \cap N$ is a lattice in $N \cap \Gamma$. Notice that this implies that $\Gamma \cap Z(N)$ is a lattice in $Z(N)$ where $Z(N)$ is the center of $N$. Recall the constant $c=\frac{1}{4(p+4 q)}$ and let $d=1 / \sqrt{c}$ if $q=0$ and $d=1 / 2 \sqrt{c}$ otherwise. We define the period $r_{\Gamma}$ of $\Gamma$ to be the positive number

$$
r_{\Gamma}=\frac{1}{d} \min \{\|\log \gamma\|: \gamma \in Z(N) \cap \Gamma, \gamma \neq \mathbf{1}, N \text { cuspidal }\} .
$$

We now fix $M A N$ and $E^{\prime} \in \log (Z(N) \cap \Gamma), E^{\prime} \neq 0$, such that $\left\|E^{\prime}\right\|$ is minimal for all possible choices of $N$. Then $\left\|E^{\prime}\right\|=d r_{\Gamma}$.

Next let $E \in \mathbb{R}^{+} E^{\prime}$ be such that for $F=\theta(E)$ and $H=[E, F]$ the set $\{H, E, F\}$ forms an $\mathfrak{s l}(2)$-triple. Recall from Remark 8.10 that $\|E\|=d$ so that

$$
E^{\prime}=r_{\Gamma} E
$$

For $y>1$ we set $a_{y}=\exp (\log y \cdot H / 2) \in A$.

We fix a Maaß cusp form $\phi$ for $\Gamma$, fix $n \in N$ and $y>1$ and consider the function

$$
F_{n, y}: \mathbb{R} \rightarrow \mathbb{C}, \quad u \mapsto \phi\left(\exp (u E) n a_{y} \cdot x_{0}\right)
$$

From the relation (10.1), it follows that $F_{n, y}$ is periodic with period $r_{\Gamma}$. Thus $F_{n, y}$ admits a Fourier expansion

$$
F_{n, y}(u)=\sum_{k \in \mathbb{Z}^{\times}} A_{k}(n, y) e^{\frac{2 \pi i k}{r_{\Gamma}} u}
$$

As $\exp (u E) \in Z(N)$ we notice next that

$$
\exp (u E) n a_{y}=n a_{y} \exp (u / y E)
$$

and we conclude with Theorem 8.11 that $F_{n, y}$ extends a holomorphic function on the strip domain $S_{y}=\{u+i v \in \mathbb{C}|| v \mid<y\}$. For $0<\epsilon<1$ we obtain, as in the previous section, the coefficient estimate

$$
\left|A_{k}(n, y)\right| \leq e^{-\frac{2 \pi y(1-\epsilon)}{r_{\Gamma}}} \sup _{\Gamma g \in \Gamma \backslash G}\left|\phi\left(\Gamma g \exp (i(1-\epsilon) E) \cdot x_{0}\right)\right| .
$$

From now on we make the slightly restrictive assumption that $\phi$ corresponds to a spherical principal series representation $\pi_{\lambda}$ with $\lambda \in i \mathfrak{a}^{*}$. Often we will identify $i \mathfrak{a}^{*}$ with $i \mathbb{R}$ via $\lambda=\lambda \cdot \rho$. 
Lemma 10.1. Let $\phi$ be a Maaß cusp form associated to $\pi_{\lambda}$. Then for all $0<\epsilon<1$ the following estimate holds:

$$
\sup _{\Gamma g \in \Gamma \backslash G}\left|\phi\left(\Gamma g \exp (i(1-\epsilon) E) \cdot x_{0}\right)\right| \leq C(\lambda) \begin{cases}|\log \epsilon|^{1 / 2} & \text { if } p=1 \text { and } q=0, \\ \epsilon^{\frac{1-p}{4}} & \text { if } p>1 \text { and } q=0, \\ |\log \epsilon|^{1 / 2} & \text { if } q=1, \\ \epsilon^{\frac{1-q}{4}} & \text { if } q>1,\end{cases}
$$

where

$$
C(\lambda)=C \cdot e^{\frac{\pi}{2}|\lambda|}(|\lambda|+1)^{1+[\operatorname{dim} X / 2]},
$$

and $C>0$ a constant independent of $\lambda$.

Proof. In first order approximation we have $G \exp (i(1-\epsilon) E) \cdot x_{0}=$ $G \exp (i(\pi / 4-2 \sqrt{\epsilon}) H) . x_{0}$ as in the proof of Lemma 9.3. Now for fixed $\lambda$, the assertion follows from [KrS1, Th. $5.1 \&$ Th. 6.17] (or alternatively from our table in Theorem 7.9). The precise shape of the constant $C(\lambda)$ is found by tracing the proofs in [KrS1].

Finally, specializing to $y=1 / \epsilon$ in (10.2) we obtain from Lemma 10.1 the following result:

Theorem 10.2. Let $\phi$ be a Maaß cusp form associated to $\pi_{\lambda}$ with $\lambda \in i \mathfrak{a}^{*}$. Then, for all $n \in N$ and $y>2$ the following estimate holds:

$$
\sup _{n \in N}\left|\phi\left(n a_{y} \cdot x_{0}\right)\right| \leq C(\lambda) e^{-\frac{2 \pi y}{r_{\Gamma}}} \begin{cases}(\log y)^{1 / 2} & \text { if } p=1 \text { and } q=0 \\ y^{\frac{p-1}{4}} & \text { if } p>1 \text { and } q=0 \\ (\log y)^{1 / 2} & \text { if } q=1 \\ y^{\frac{q-1}{4}} & \text { if } q>1\end{cases}
$$

\section{Exponential Decay of Maaß Cusp Forms III: The Higher-Rank Cases}

Throughout this section we denote by $G$ a simple Lie group and by $\Gamma<G$ a noncocompact lattice. We say that a parabolic subgroup $P=M A N$ is cuspidal if $\Gamma_{N}=\Gamma \cap N$ is a lattice in $N$.

Definition 11.1. A $\Gamma$-invariant smooth $\mathbb{D}(X)$-eigenfunction on $X$ is called a weak Maaß automorphic form. A weekly automorphic Maaß form is called a Maaß cusp form if it is of moderate growth and

$$
\int_{\Gamma_{N} \backslash N} f\left(\Gamma_{N} n g\right) d\left(\Gamma_{N} n\right)=0
$$

for all $g \in G$ and all proper cuspidal parabolic subgroups $P=M A N$. 
REMARK 11.2. The crucial fact for us is that all $\mathbb{D}(X)$-eigenfunctions on $X$ extend holomorphically to $\Xi$ (cf. [KrS2, Th. 1.1]). Hence all weak Maaß automorphic forms extend to holomorphic functions on $\Xi$. If moreover $\Gamma$ is torsion free, then $\Gamma$ acts properly on $\Xi$ (as the $G$-action is proper) and we can form the quotient $\Gamma \backslash \Xi$ in the category of complex manifolds. Thus Maaß forms have $\Gamma \backslash \Xi$ as their natural domain of definition.

For the rest of this section we let $P=M A N$ be a minimal parabolic subgroup which is cuspidal. In addition we make the following

Assumption. For each root $\alpha \in \Sigma^{+}$the group $\Gamma \cap \exp \left(\mathfrak{g}^{\alpha}\right)$ is a lattice in $\exp \left(\mathfrak{g}^{\alpha}\right)$.

For each $\alpha \in \Sigma^{+}$and $E_{\alpha} \in \mathfrak{g}^{\alpha}$ we set $F_{\alpha}=\theta\left(E_{\alpha}\right)$ and $H_{\alpha}=\left[E_{\alpha}, F_{\alpha}\right]$. We always normalize $E_{\alpha}$ in such a way that $\left\{E_{\alpha}, F_{\alpha}, H_{\alpha}\right\}$ forms an $\mathfrak{s l}(2)$ triplet.

For $\alpha \in \Pi$ we define an ideal in $\Sigma$ by

$$
\Sigma_{\alpha}=\left\{\beta=\sum_{\gamma \in \Pi} n_{\gamma} \gamma \mid n_{\alpha}>0\right\} \subset \Sigma^{+},
$$

and write $\mathfrak{u}_{\alpha}=\bigoplus_{\beta \in \Sigma_{\alpha}} \mathfrak{g}^{\beta}$ for the corresponding ideal in $\mathfrak{n}$. We set $U_{\alpha}=$ $\exp \left(\mathfrak{u}_{\alpha}\right)$ and notice that $U_{\alpha}$ is the nilradical of the cuspidal parabolic subgroup $P_{\alpha}$ attached to $\alpha \in \Pi$.

Associated to $\alpha \in \Pi$ we define positive constants

$$
r_{\alpha, \Gamma}=\max _{\beta \in \Sigma_{\alpha}} \min \left\{c>0 \mid \exp \left(c E_{\beta}\right) \in \Gamma, E_{\beta} \in \mathfrak{g}^{\beta} \text { normalized }\right\},
$$

and

$$
c_{\alpha}=\min \left\{c>0 \mid \frac{c}{2} H_{\beta} \in \partial \Omega, \beta \in \Sigma_{\alpha}\right\} .
$$

REMARK 11.3. The relevance of the number $c_{\alpha}$ is the following: it is the maximal number such that $\exp \left(i t E_{\beta}\right) \cdot x_{0} \in \Xi$ for all $0 \leq t<c_{\alpha}$ and $\beta \in \Sigma_{\alpha}$.

For a subgroup $U<N$ with $\Gamma_{U} \backslash U$ and compact we define the constant term of a function $f \in C^{\infty}\left(\Gamma_{N} \backslash G\right)$ with respect to $U$ as

$$
\pi_{U} f(U g)=\int_{U_{N} \backslash U} f\left(\Gamma_{N} u g\right) d\left(U_{N} u\right) .
$$

Note that $\pi_{U} f \in C^{\infty}\left(\Gamma_{N} U \backslash G\right)$. For $U=U_{\alpha}$ we use the simplifying notation $\pi_{\alpha}=\pi_{U_{\alpha}}$ for the constant term with respect to $U_{\alpha}$.

We can now state the holomorphic analog of the Main Lemma (Lemma 10) in [H2].

Lemma 11.4 (Main Lemma). Let $\alpha \in \Pi$ and $0<\epsilon<1$. Let $f \in C^{\infty}\left(\Gamma_{N} \backslash G\right)$ such that $f$ admits a holomorphic continuation to $\Gamma_{N} \backslash \tilde{\Xi}$. Then there exists 
a constant $C_{\alpha}>0$, only depending on $\alpha$, such that

$$
\left|\left(f-\pi_{\alpha} f\right)\left(\Gamma_{N} a\right)\right| \leq C_{\alpha} e^{-\frac{a^{\alpha}(1-\epsilon)}{r_{\alpha, \Gamma}}} \cdot \sup _{\substack{g \in G \\ \beta \in \Sigma_{\alpha}}}\left|f\left(\Gamma_{N} g \exp \left(i(1-\epsilon) c_{\alpha} E_{\beta}\right)\right)\right|
$$

for all $a \in A^{+}$.

Proof. We follow [H2, Ch. I, $\S 7]$. We order the roots of $\Sigma_{\alpha}$, say

$$
\beta_{1}>\beta_{2}>\ldots>\beta_{s}
$$

and form ideals of $\mathfrak{n}$ by

$$
\mathfrak{n}_{i}=\bigoplus_{j=1}^{i} \mathfrak{g}^{\beta_{j}} \quad(0 \leq i \leq s)
$$

Set $U_{i}=\exp \left(\mathfrak{n}_{i}\right)$. Note that $\Gamma \cap U_{i}$ is cocompact in $U_{i}$ by our assumption on $\Gamma$. Thus $\pi_{U_{i}}$ is defined. Note that $\pi_{U_{0}}=\mathrm{id}$ and $\pi_{U_{s}}=\pi_{\alpha}$. We now verify the stronger statement (cf. [H2, Lem. 19]):

$$
f-\pi_{U_{j}} f \text { satisfies (11.1). }
$$

We prove (11.2) by induction, following the arguments for the proof of [H2, Lem. 19]. The case $i=0$ is clear. Notice that $\mathfrak{u}_{i}=\mathfrak{u}_{i-1} \oplus \mathfrak{g}^{\beta_{i}}$. Choose a basis $E_{1}, \ldots, E_{p}$ of $\mathfrak{g}^{\beta_{i}}$ of normalized elements. We require in addition that $\exp \left(r_{\alpha, \Gamma} E_{j}\right) \in \Gamma_{N}$. For $0 \leq j \leq p$ we set

$$
\mathfrak{v}_{j}=\mathfrak{u}_{i} \oplus \bigoplus_{k=1}^{j} \mathbb{R} E_{k} \quad \text { and } \quad V_{j}=\exp \left(\mathfrak{v}_{j}\right) .
$$

Observe that each $V_{j}$ is a normal subgroup of $N$ with $\Gamma \cap V_{j}<V_{j}$ cocompact. In particular $\pi_{V_{j}}$ is defined. Set $\phi_{f, j}=\pi_{V_{j}} f$ for $0 \leq j \leq p$ and

$$
\psi_{f, j}=\phi_{f, j-1}-\phi_{f, j} \quad(1 \leq j \leq p) .
$$

Then

$$
\pi_{U_{i-1}} f-\pi_{U_{i}} f=\phi_{f, 0}-\phi_{f, p}=\sum_{j=1}^{p} \psi_{f, j}
$$

and

$$
f-\pi_{U_{j}} f=\sum_{i=1}^{j} \pi_{U_{i-1}} f-\pi_{U_{i}} f
$$

imply that it is sufficient to establish for all $1 \leq j \leq p$ (cf. [H2, Lem. 20]):

$$
\psi_{f, j} \text { satisfies (11.1). }
$$

For that let us fix $j$ and write $\phi_{f}=\phi_{f, j-1}, V=V_{j}$. So $\phi_{f}=\pi_{V} f$. Consider the mapping

$$
V_{j-1} \backslash V_{j} \rightarrow \mathbb{C}, \quad \mapsto \phi_{f}(v a),
$$


and note that this function is left invariant under $\Gamma \cap V_{j} . \operatorname{As} \exp \left(r_{\alpha, \Gamma} E_{j}\right) \in$ $\Gamma \cap V_{j}$ we obtain that

$\phi_{f}(a)=\sum_{q \in \mathbb{Z}} \vartheta_{f, q}(a) \quad$ where $\quad \vartheta_{f, q}(a)=\frac{1}{r_{\alpha, \Gamma}} \int_{0}^{r_{\alpha, \Gamma}} \phi_{f}\left(\exp \left(t E_{j}\right) a\right) e^{-\frac{2 \pi i q t}{r_{\alpha, \Gamma}}} d t$.

We fix $a \in A_{+}, q \in \mathbb{Z}$ and consider the function

$$
F_{f, q}(z)=\phi_{f}\left(\exp \left(z E_{j}\right) a\right)=\phi_{f}\left(a \exp \left(a^{-\beta_{j}} z E_{j}\right)\right)
$$

for $z \in \mathbb{R}$. We conclude that $F_{f, q}$ admits a holomorphic continuation to the strip domain

$$
S=\left\{z \in \mathbb{C}|| \operatorname{Im} z \mid<c_{\alpha} \cdot a^{\beta_{j}}\right\} .
$$

Thus, as in the preceding two sections, we obtain for $0<\epsilon<1$ the estimate

$$
\left|\theta_{f, q}(a)\right| \leq M_{\epsilon} \cdot e^{\frac{-2 \pi q a^{\beta} \beta_{j}(1-\epsilon) c_{\alpha}}{r_{\alpha, \Gamma}}}
$$

where

$$
M_{\epsilon}=\sup _{g \in G} \mid f\left(\Gamma_{N} g \exp \left(i(1-\epsilon) c_{\alpha} E_{j}\right) \mid .\right.
$$

We sum up the geometric series and note that $\vartheta_{f, 0}=\phi_{f, j}$ and obtain the desired estimate for $\psi_{f, j}=\phi_{f}-\phi_{f, j}$. This proves the lemma.

This lemma has an an immediate consequence the following important result (compare to [H2, Cor. to Lem. 10]).

Corollary 11.5 (Main Estimate). Suppose that $f$ is Maaß cusp form. Then there exist a constant $C>0$, independent from $f$, such that for all $a \in A^{+}$

$$
|f(\Gamma a)| \leq C \cdot \min _{\alpha \in \Pi} e^{-\frac{2 \pi(1-\epsilon) a^{\alpha} c_{\alpha}}{r_{\alpha, \Gamma}}} \cdot M_{\epsilon}
$$

with

$$
M_{\epsilon}=\sup _{g \in G} \sup _{\substack{\beta \in \Sigma_{\alpha} \\ \alpha \in \Pi}} \mid f\left(\Gamma g \exp \left(i(1-\epsilon) c_{\alpha} E_{\beta}\right) \mid .\right.
$$

EXAMPLE 11.6. It is instructive to consider the following example

$$
G=\operatorname{Sl}(n, \mathbb{R}) \quad \text { and } \quad \Gamma=\operatorname{Sl}(n, \mathbb{Z}) .
$$

In this situation we have $r_{\alpha, \Gamma}=c_{\alpha}=1$ for all $\alpha$ and the estimate in the corollary becomes

$$
|f(\Gamma a)| \leq C \cdot e^{-2 \pi(1-\epsilon) \cdot \max _{1 \leq i \leq n-1} \frac{a_{i}}{a_{i+1}}} \cdot M_{\epsilon}
$$

with

$$
M_{\epsilon}=\sup _{g \in G} \sup _{1 \leq i<j \leq n}\left|f\left(\Gamma g \exp \left(i(1-\epsilon) E_{i j}\right)\right)\right| .
$$


11.1 Refinements of the main estimate. It is possible to do a little bit better as in the main estimate once we apply the more refined geometric results from subsections 8.2.2 and 8.3. To state the inequalities in a more compact form we define

$$
r_{\Gamma}=\sup _{\alpha} r_{\alpha, \Gamma}
$$

We begin with the Hermitian cases, i.e. where $\Sigma$ is of type $C_{n}$. We restrict our attention to the maximal Siegel parabolic with abelian nilradical and proceed as in Lemma 11.4 going simultaneously in the direction of the strongly orthogonal $E_{j}$ (cf. the notation in subsection 8.2.2). Then Theorem 8.15 gives the following result.

Lemma 11.7 (Main Estimate refined - the Hermitian case). Suppose that $\Xi$ is a Hermitian symmetric space and $f$ is a Maaß cusp form on $X$. Then, with the notation of subsection 8.2.2, there exists a constant $C>0$, independent from $f$, such that for all $t_{i} \geq 0$

$$
\left|f\left(\Gamma \exp \left(\sum_{j=1}^{n} t_{j} T_{j}\right)\right)\right| \leq C \cdot e^{-\frac{2 \pi(1-\epsilon)\left(\sum_{j=1}^{n} t_{j}\right)}{r_{\Gamma}}} \cdot M_{\epsilon}
$$

with

$$
\left.M_{\epsilon}=\sup _{g \in G} \mid f\left(\Gamma g \exp \left(i(1-\epsilon) \sum_{j=1}^{n} E_{j}\right)\right)\right) \mid .
$$

Finally we draw attention to the case of $G=\operatorname{Sl}(n, \mathbb{R})$ and our fine geometric results in subsection 8.3. We will state our result for the Whittaker functionals of a Maaß cusp form. It is no loss of generality to assume that $N$, the group of unipotent upper triangular matrices, is cuspidal for the lattice $\Gamma$. Let us fix a unitary character $\chi: N \rightarrow \mathbb{S}^{1}$. As $\chi$ is necessarily trivial on $[N, N]$, it is clear that $\chi$ is given by a parameter $\mathbf{m}=\left(m_{1}, \ldots, m_{n-1}\right) \in \mathbb{R}^{n-1}$, namely

$$
\chi\left(\begin{array}{ccccc}
1 & t_{1} & * & \ldots & * \\
& \ddots & \ddots & * & * \\
& & 1 & t_{n-1} & * \\
& & & & 1
\end{array}\right)=e^{2 \pi i \sum_{j=1}^{n-1} t_{j} m_{j}} .
$$

In the sequel we assume that $\chi$ is trivial on $\Gamma_{N}$.

For a cusp form $f$ we then define the Whittaker function with respect to $\chi$ by

$$
W(f, \chi)(g)=\int_{\Gamma_{N} \backslash N} f(\Gamma n g) \chi(n) d\left(\Gamma_{N} n\right) \quad(g \in G),
$$

and note that $W(f, \chi) \in C^{\infty}(G / N, \chi)$. The obvious application of our standard technique yields 
Lemma 11.8 (Main Estimate refined - Whittaker functionals for the special linear group). Let $G=\operatorname{Sl}(n, \mathbb{R})$ and $f$ be a Maaß cusp form on $X$. Then, with the notation of subsection 8.3, there exists a constant $C>0$, independent from $f$, such that for all $a=\operatorname{diag}\left(a_{1}, \ldots, a_{n}\right) \in A^{+}$

with

$$
|W(f, \chi)(a)| \leq C \cdot e^{-\frac{2 \pi(1-\epsilon)}{r_{\Gamma}} \sum_{j=1}^{n-1}\left|m_{j}\right| \cdot \frac{a_{j}}{a_{j+1}}} \cdot M_{\epsilon}
$$

$$
M_{\epsilon}=\sup _{g \in G}|f(\Gamma g z(1-\epsilon))| .
$$

11.2 A Bergman estimate on the local crown domains. To proceed with our estimates on Maaß cusp forms we need to control the quantities

$$
M_{\epsilon}=\sup _{g \in G}\left|f\left(\gamma g n_{\epsilon}\right)\right|
$$

for certain $n_{\epsilon} \in N_{\mathbb{C}}$. In order to do so we estimate $M_{\epsilon}$ against an $L^{2}$-norm, which can be controlled in terms of representation theory.

We state the result.

Proposition 11.9. Let $\Gamma$ be a lattice in the semi-simple group $G$. Fix an element $Z_{0} \in \partial \pi \Omega / 2$ and a constant $0<\epsilon<1$. Let $f$ be a $\Gamma$ invariant holomorphic function on $\Xi$. Then there exists a constant $C>0$, independent of $f$, such that for all $g \in G$

$$
\begin{aligned}
& \left|f\left(\Gamma g \exp \left(i(1-\epsilon) Z_{0}\right) \cdot x_{0}\right)\right| \leq C \cdot \epsilon^{-\operatorname{dim} X+\frac{1}{2} \operatorname{rank} X+\frac{1}{2} .} \\
& \cdot \sup _{\left\{Z \in \Omega \mid\left\|Z-(1-\epsilon) Z_{0}\right\|<\epsilon / 2\right\}}\left(\int_{\Gamma \backslash G}\left|f\left(\Gamma g \exp (i Z) \cdot x_{0}\right)\right|^{2} d(\Gamma g)\right)^{1 / 2} .
\end{aligned}
$$

Before we start with the proof let us recall the basic Bergman estimate for polydiscs in $\mathbb{C}^{n}$. Fix $z_{0} \in \mathbb{C}^{n}$. For $r>0$ let us define the polydisc centered at $z_{0}$ with radius $r$ by

$$
P\left(z_{0}, r\right)=\left\{z \in \mathbb{C}^{n} \mid\left\|z-z_{0}\right\|_{\infty}<r\right\} .
$$

One expands a holomorphic function $f \in \mathcal{O}\left(P\left(z_{0}, r\right)\right)$ in a power series at $z_{0}$, and uses orthogonality of the monomials; the result is the Bergman estimate

$$
\left|f\left(z_{0}\right)\right| \leq \frac{1}{\pi^{n} \cdot r^{n}} \cdot\|f\|_{L^{2}\left(P\left(z_{0}, r\right)\right)} .
$$

We turn to the proof of the proposition.

Proof. We normalize the Killing norm $\|\cdot\|$ on $\mathfrak{p}$ such that $\left\|Z_{0}\right\|=1$. Let $Z_{\epsilon}=(1-\epsilon) Z_{0}$. We define various balls in $\mathfrak{p}$ and $\mathfrak{p}_{\mathbb{C}}$ :

$$
\begin{aligned}
& B_{1}=\left\{U \in \mathfrak{p} \mid\left\|U-Z_{\epsilon}\right\|<\epsilon / 2\right\}, \\
& B_{2}=\{V \in \mathfrak{p} \mid\|V\|<\epsilon / 2\},
\end{aligned}
$$




$$
B=\left\{Z=U+i V \mid U \in B_{1}, V \in B_{2}\right\} .
$$

If necessary we may replace $\epsilon$ by $c \epsilon$ for some positive constant in the definition of $B_{1}$ and henceforth assume that $B_{1} \subset \pi \hat{\Omega} / 2$. Then it is clear that $\exp \left(B_{2}\right) \exp \left(B_{1}\right) \cdot x_{0} \subset \Xi$, but what about $\exp (B) \cdot x_{0}$ ? This is not clear, but after some controlled shrinking we are in good shape:

Lemma 11.10. There exists $c>0$ such that for all $0<\epsilon<1$

$$
\exp \left(B_{2}\right) \exp \left(i B_{1}\right) \cdot x_{0} \supset \exp \left(\left\{Z=U+i V \in \mathfrak{p}_{\mathbb{C}}:\left\|Z-Z_{\epsilon}\right\|<c \epsilon / 2\right\}\right) .
$$

Proof. We remark that $\pi \hat{\Omega} / 2$ is compact and that

$$
d \exp (i Z): \mathfrak{p}_{\mathbb{C}} \rightarrow T_{\exp (i Z) \cdot x_{0}} X_{\mathbb{C}}
$$

is invertible for all $Z \in \pi \overline{\hat{\Omega}} / 2$. In fact, the Jacobian of exp at $i Z$ is given by

$$
|\operatorname{det} d \exp (i Z)|=\left|\prod_{\alpha \in \Sigma^{+}} \frac{\sinh \alpha(i Z)}{\alpha(i Z)}\right| \text {. }
$$

The assertion follows from the implicit function theorem.

It is no loss of generality to assume that the constant $c$ in the previous lemma is 1. At any rate the previous lemma combined with the Bergman estimate yields

$$
\left|\phi\left(\exp \left(i Z_{\epsilon}\right) \cdot x_{0}\right)\right| \leq C \cdot \frac{1}{\epsilon^{\operatorname{dim} X}}\left(\int_{\exp \left(B_{2}\right) \exp \left(i B_{1}\right) \cdot x_{0}} \|\left.\phi(z)\right|^{2} d z\right)^{1 / 2}
$$

for a constant $C>0$ and all functions $\phi \in \mathcal{O}(\Xi)$. Here, $d z$ denotes the Haar measure on $X_{\mathbb{C}}$.

Next we set $B_{\mathfrak{a}, 1}=B_{1} \cap \mathfrak{a}$ and note that

$$
\operatorname{Ad}(K) B_{\mathfrak{a}, 1} \supseteq B_{1}^{\prime}
$$

with $B_{1}^{\prime}=\left\{U \in \mathfrak{p} \mid\left\|U-Z_{\epsilon}\right\|<c \epsilon / 2\right\}$ for some constant $c>0$. Again it is no loss of generality to assume that $B_{1}^{\prime}=B_{1}$. As a consequence we derive from (11.11) that

$$
\left|\phi\left(\exp \left(i Z_{\epsilon}\right) \cdot x_{0}\right)\right| \leq C \cdot \frac{1}{\epsilon^{\operatorname{dim} X}}\left(\int_{\Gamma \backslash G \exp \left(i B_{1}\right) \cdot x_{0}}|\phi(z)|^{2} d z\right)^{1 / 2}
$$

for all $\Gamma$-invariant holomorphic functions $\phi$ on $\Xi$. Finally we use the integration formula [KrS2, Prop.,4.6], and obtain with

$$
\int_{\Gamma \backslash G \exp \left(i B_{1}\right) \cdot x_{0}}^{\text {that }}|\phi(z)|^{2} d z=\int_{\Gamma \backslash G} \int_{B_{\mathfrak{a}, 1}}\left|\phi\left(\Gamma g \exp (i Y) \cdot x_{0}\right)\right|^{2} \cdot J(Y) d(\Gamma g) d Y \text {. }
$$

$$
J(Y)=\prod_{\alpha \in \Sigma^{+}}|\sin 2 \alpha(Y)|^{m_{\alpha}} \quad(Y \in \mathfrak{a})
$$


Notice that $J(Y) \leq 2 \epsilon$ as at least one root is going to vanish on $Z_{\epsilon}$ for $\epsilon \rightarrow 0$. Thus after combining (11.12) and (11.13) we obtain for all $g \in G$ that

$$
\begin{aligned}
\left|f\left(g \exp \left(i Z_{\epsilon}\right) \cdot x_{0}\right)\right| \leq C \cdot \epsilon^{-\operatorname{dim} X+\frac{1}{2}(1+\operatorname{rank} X)} & \cdot \sup _{Y \in B_{\mathfrak{a}, 1}}\left(\int_{\Gamma \backslash G} f\left(\left.\Gamma g \exp (i Y) \cdot x_{0}\right|^{2} d z\right)^{1 / 2}\right.
\end{aligned}
$$

for all $\Gamma$-invariant $f \in \mathcal{O}(\Xi)$.

11.3 Main estimates in final form. In this concluding subsection we put our previously obtained results together in order to obtain final version of our main estimates Corollary 11.5, Lemma 11.7 and Lemma 11.8. The main task is to obtain estimates for the quantities $M_{\epsilon}$ in (11.5), (11.7) and (11.9). We give details for the main case in (11.5), and confine ourselves to stating the analogous results for the remaining two cases. So we wish to control the behavior of

$$
M_{\epsilon}=\sup _{g \in G} \sup _{\substack{\beta \in \Sigma_{\alpha} \\ \alpha \in \Pi}} \mid f\left(\Gamma g \exp \left(i(1-\epsilon) c_{\alpha} E_{\beta}\right) \mid .\right.
$$

First we deduce from Lemma 3.3 that

Set

$$
M_{\epsilon} \leq \sup _{g \in G} \sup _{Y \in \partial \Omega} \mid f(\Gamma g \exp (i(1-2 \sqrt{\epsilon}) \pi Y / 2) \mid .
$$

$$
r^{X}:=-\operatorname{dim} X+\frac{1}{2} \operatorname{rank} X+\frac{1}{2} .
$$

Then it follows from Proposition 11.9 and (11.14) that

$$
M_{\epsilon} \leq C \cdot \epsilon^{r^{X} / 2} \sup _{Y \in \partial \Omega} \cdot\left(\int_{\Gamma \backslash G}\left|f\left(\Gamma g \exp (i(1-c \sqrt{\epsilon}) \pi Y / 2) \cdot x_{0}\right)\right|^{2} d(\Gamma g)\right)^{1 / 2}
$$

for constants $C, c>0$ only depending on $X$. Assume that $f$ corresponds to the spherical representation $\pi_{\mu}$. Recall the exponents $s^{X}$ and $d^{X}$ from (7.4). Now, Theorem 7.3 applies and we arrive at

$$
M_{\epsilon} \leq C(\mu) \cdot \epsilon^{r^{X} / 2+s^{X} / 4}|\log \epsilon|^{d^{X} / 2}
$$

for a constant $C=C(\mu)$ depending on $\mu$ and the geometry of $X$. If we specialize in Corollary 11.5 to $\epsilon=\min _{\alpha \in \Pi} a^{-\alpha}$ we get the following from (11.16):

Theorem 11.11 (Main estimate). Suppose that $f$ is Maaß cusp form corresponding to $\pi_{\mu}$. Then there exist a constant $C=C(\mu)>0$ such that for all $a \in A^{+}$

$$
|f(\Gamma a)| \leq C \cdot \min _{\alpha \in \Pi} e^{-\frac{2 \pi a^{\alpha} c_{\alpha}}{r_{\alpha, \Gamma}}} \cdot \max _{\alpha \in \Pi} a^{-\alpha\left(r^{X} / 2+s^{X} / 4\right)} \cdot|\alpha(\log a)|^{d^{X} / 2} .
$$


In similar manner we obtain a more concrete version of Lemma 11.7:

Theorem 11.12 (Main estimate refined - the Hermitian case). Suppose that $\Xi$ is a Hermitian symmetric space and $f$ is a Maaß cusp form on $X$ corresponding to $\pi_{\mu}$. Then, with the notation of subsection 8.2.2, there exists a constant $C=C(\mu)>0$ such that for all $t_{i} \geq 0$

$$
\left|f\left(\Gamma \exp \left(\sum_{j=1}^{n} t_{j} T_{j}\right)\right)\right| \leq C \cdot e^{-\frac{2 \pi\left(\sum_{j=1}^{n} t_{j}\right)}{r_{\Gamma}}} \cdot\left(\sum_{j=1}^{n} t_{j}\right)^{-r^{X} / 2-s^{X} / 4} .
$$

Finally we state a new version of Lemma 11.8 (for which one also needs to employ the estimate in Proposition 8.18):

Theorem 11.13 (Main estimate refined - Whittaker functionals for the special linear group). Let $G=\operatorname{Sl}(n, \mathbb{R})$ and $f$ be a Maaß cusp form on $X$ corresponding to $\pi_{\mu}$. Then there exists a constant $C=C(\mu)$ such that for all $a=\operatorname{diag}\left(a_{1}, \ldots, a_{n}\right) \in A^{+}$

$$
\begin{array}{r}
|W(f, \chi)(a)| \leq C \cdot e^{-\frac{2 \pi}{r_{\Gamma}}\left(\sum_{j=1}^{n-1}\left|m_{j}\right| \cdot \frac{a_{j}}{a_{j+1}}\right)} \cdot\left(\sum_{j=1}^{n-1}\left|m_{j}\right| \cdot \frac{a_{j}}{a_{j+1}}\right)^{-r^{X}-s^{X} / 2} \\
\cdot\left|\log \left(\sum_{j=1}^{n-1}\left|m_{j}\right| \cdot \frac{a_{j}}{a_{j+1}}\right)\right| .
\end{array}
$$

REMARK 11.14 (Some generalizations of the main estimates). Let $F$ either denote a Maaß cusp form $f$ or a Whittaker function $W(f, \chi)$ in case $G=$ $\operatorname{Sl}(n, \mathbb{R})$. The general form of our estimates in Theorems 11.11, 11.12, 11.13, then is

$$
\left(\forall a \in A^{+}\right) \quad|F(\Gamma a)| \leq C e^{-\phi(a)} \cdot P(a)
$$

where $C>0$ is a constant only depending on the representation $\pi_{\mu}$ associated to $F$,

$$
\phi(a)=\sum_{\alpha \in \Sigma^{+}} c_{\alpha} a^{\alpha} \quad\left(c_{\alpha} \geq 0\right)
$$

is a "positive" linear functional and $P(a)$ is a polynomial in the variables $a^{\alpha}, \alpha(\log a)$ with $\alpha \in \Sigma^{+}$.

(a) (Extension to a Siegel domain) We restricted ourselves to estimates on $A^{+}$. However, for certain applications in number theory one needs estimates which are uniform on a Siegel domain

$$
\mathfrak{S}_{t}=\omega A_{t} K \quad(t>1)
$$

where $\omega \subset N$ is a fixed compact and $A_{t}=\left\{a \in A \mid a^{\alpha}>t \forall \alpha \in \Pi\right\}$. The version of (11.19) on the whole Siegel domain $\mathfrak{S}_{t}$ is

$$
(\forall n \in \omega)\left(\forall a \in A_{t}\right) \quad|F(\Gamma n a)| \leq C_{t} e^{-c_{t} \phi(a)} \cdot P(a)
$$


where $c_{t}, C_{t}>0$ are such that $c_{t} \rightarrow 1^{-}$for $t \rightarrow \infty$. Let us explain how this is derived from (11.19). For the proof of (11.19) we use certain subsets $\Lambda_{0} \subset \Lambda$ and applied the fact that $F$ extends to a function on $\Gamma \exp \left(i \operatorname{Ad}(a) \Lambda_{0}\right) a . x_{0}$; recall, the precise rate of exponential decay was directly linked to the geometry of $\Lambda_{0}$. If one wants estimates on $\mathfrak{S}_{t}$ one needs to bring in $\omega$ variables, i.e. we look for maximal subsets $\Lambda_{t} \subset \Lambda_{0}$ such that $F$ extends to $\Gamma \exp \left(i \operatorname{Ad}(a) \Lambda_{t}\right) \omega a \cdot x_{0}$. This is equivalent to the requirement of

$$
\exp \left(i \Lambda_{t}\right) a \omega a^{-1} \cdot x_{0} \subset \Xi \text {. }
$$

Now $a \omega a^{-1}$ shrinks to $\{\mathbf{1}\}$ for $t \rightarrow \infty$, meaning $\bigcup_{t>0} \Lambda_{t}=\Lambda_{0}$.

(b) (Extension to other $K$-types) We only considered Maaß cusp forms, i.e. cusp forms associated to a trivial $K$-type. However, it is possible to extend to other $K$-types $\sigma \in \hat{K}$. For that one needs uniform quantitative control of the projection $\kappa: N_{\mathbb{C}} A_{\mathbb{C}} K_{\mathbb{C}} \rightarrow K_{\mathbb{C}}$ on $K$-orbits through $\exp (i \pi \Omega / 2)$. This will be deferred to another paper. In any case, the result then is

$$
\left(\forall n a k \in \mathfrak{S}_{t}\right) \quad \mid F(\text { Гnak }) \mid \leq C_{t} e^{-c_{t} \phi(a)} \cdot P_{\sigma}(a)
$$

with $C_{t}=C_{t}(\mu, \sigma)$ and $P=P_{\sigma}$ now depending on $\sigma$. Further explanation is given in item (c) below.

(c) (Extension to non-spherical representations) So far we only considered spherical representations $\pi=\pi_{\mu}$. But estimate (11.21) remains true for an arbitrary irreducible unitary representation $\pi$ and arbitrary $K$-types $\sigma$. Here is the reason. We can embed $\pi$ into a principal series representation induced off a minimal parabolic (subrepresentation theorem). One uses the fact that the smooth structures are unique (CasselmanWallach). Now for a principal series one can look at the corresponding Eisenstein integrals for the $K$-types (Harish-Chandra) and everything boils down to estimate the spherical function and the sup-norm of a holomorphically extended $K$-type (for that one needs the quantitative control of $\kappa$ ). The other details can be found in the proof of [KrS1, Th. 3.1].

REMARK 11.15 (Quality of the estimates). The rate of exponential decay given in above three theorems are sharp. We provide some evidence in the section below. Concerning the polynomial part, one could likely replace $r^{X}$ by zero. However that would require to prove Conjecture $\mathrm{C}$ in [KrS1]; something which is out of reach with currently available techniques.

REMARK 11.16 (Applications to automorphic forms). (a) ( $L$-functions) For various reasons one wants to know whether certain automorphic $L$-functions are meromorphic of finite order. For instance this information is required if one wants to exhibit zero-free regions (in the spirit of de la Vallée Poussin) 
for those $L$-functions. We refer to [GLS], [GL], [GS] for results in this direction. We wish to point out that our estimates help to establish that $L$-functions with appropriate integral representations are in fact of finite order.

(b) (Voronoi summation) Recently Voronoi summation was established for $\operatorname{Gl}(3, \mathbb{R})$, cf. [MilS]. Shortly after it was extended to $\operatorname{Gl}(n, \mathbb{R})$ in $[\mathrm{GoL}]$. In the approach of [GoL] it becomes visible that exponential decay is an important analytical ingredient to establish Voronoi summation.

\section{Final Remarks}

12.1 Estimates on Whittaker functionals for $\operatorname{Gl}(n)$ are sharp. We show that the rate of exponential decay for Whittaker functionals for $G=\mathrm{Gl}(n, \mathbb{R})$ proved in Theorem 11.13 is optimal.

To begin with we recall the Whittaker expansion of Piatetski-Shapiro and Shalika for a cuspidal Maaß form of the group $\operatorname{Gl}(n, \mathbb{R})$. For simplicity let us restrict ourselves to the case $n=3$. Our arithmetic subgroup of choice will be $\Gamma=\operatorname{Gl}(3, \mathbb{Z})$. Let us define subgroups of $\Gamma$ by

$$
\Gamma^{2}=\left(\begin{array}{cc}
\operatorname{Gl}(2, \mathbb{Z}) & 0 \\
0 & 1
\end{array}\right) \quad \text { and } \quad \Gamma_{N}^{2}=\Gamma^{2} \cap N
$$

In the sequel we use the notation introduced in subsection 11.1. For $\chi$ corresponding to $\mathbf{m}=(1,1)$ and $f$ a Maaß cusp form we set $W(f)=$ $W(f, \chi)$. For $n_{1}, n_{2} \in \mathbb{N}$ we put

$$
W_{n_{1}, n_{2}}(f)(z)=W(f)\left(\left(\begin{array}{lll}
n_{1} n_{2} & & \\
& n_{1} & \\
& & 1
\end{array}\right) z\right)
$$

where $z \in X=\operatorname{Gl}(3, \mathbb{R}) / \mathrm{O}(3, \mathbb{R})$. The Whittaker expansion of $f$ reads as

$$
f(z)=\sum_{\gamma \in \Gamma_{N}^{2} \backslash \Gamma^{2}} \sum_{n, 1, n_{2} \in \mathbb{N}} \frac{a_{n_{1}, n_{2}}}{n_{1} n_{2}} \cdot W_{n_{1}, n_{2}}(f)(z)
$$

for complex coefficients $a_{n_{1}, n_{2}}$, [Sh, Th. 5.9]. We normalize $f$ such that $a_{1,1}=1$ and draw our attention to the main result in $[\mathrm{Bu},(10.1)]$, which gives a formula for the Mellin transform of $W(f)$ :

$$
\begin{aligned}
& \int_{0}^{\infty} \int_{0}^{\infty} W\left(\begin{array}{lll}
y_{1} y_{2} & & \\
& y_{1} & \\
& & 1
\end{array}\right) \cdot y_{1}^{s_{1}-1} \cdot y_{2}^{s_{2}-1} \frac{d y_{1}}{y_{1}} \frac{d y_{2}}{y_{2}} \\
= & \frac{1}{4} \pi^{-s_{1}-s_{2}} \cdot \frac{\Gamma\left(\frac{s_{1}+\alpha}{2}\right) \Gamma\left(\frac{s_{1}+\beta}{2}\right) \Gamma\left(\frac{s_{1}+\gamma}{2}\right) \cdot \Gamma\left(\frac{s_{2}-\alpha}{2}\right) \Gamma\left(\frac{s_{2}-\beta}{2}\right) \Gamma\left(\frac{s_{2}-\gamma}{2}\right)}{\Gamma\left(\frac{s_{1}+s_{2}}{2}\right)} .
\end{aligned}
$$


Here $s_{1}, s_{2}$ are sufficiently large real numbers and $\alpha, \beta, \gamma=-\alpha-\beta$ are complex numbers related to the parameter of the principal series representation associated to $f$ (see [Bu, p. 161]). We perform a Stirling approximation of the right-hand side (RHS) of (12.1) and obtain

$$
(R H S)\left(s_{1}, s_{2}\right) \sim \frac{1}{4} \pi^{-s_{1}-s_{2}} \cdot \sqrt{2 \pi}^{5} \cdot e^{-\left(s_{1}+s_{2}\right)} \cdot \frac{\left(\frac{s_{1}}{2}\right)^{3\left(\frac{s_{1}}{2}-\frac{1}{2}\right)}\left(\frac{s_{2}}{2}\right)^{3\left(\frac{s_{2}}{2}-\frac{1}{2}\right)}}{\left(\frac{s_{1}+s_{2}}{2}\right)^{\left(\frac{s_{1}+s_{2}}{2}-\frac{1}{2}\right)}} .
$$

We specialize to $s_{1}=s_{2}=s$ and get the simpler expression

$$
(R H S)(s, s) \sim \frac{1}{2}(2 \pi)^{-2 s+5 / 2} \cdot e^{-2 s} \cdot \frac{s^{2 s-1 / 2}}{2^{s}} .
$$

Similarly, if we keep one variable fixed to be zero we get

$$
(R H S)(s, 0)=R H S(0, s) \sim C(2 \pi)^{-s} \cdot e^{-s} \cdot s^{s-1} .
$$

We wish to compare these asymptotics with what we obtain by applying the estimate for $W(f)$ from Theorem 11.13 With $N>3$ we get

$$
\left|W(f)\left(\begin{array}{ccc}
y_{1} y_{2} & & \\
& y_{1} & \\
& & 1
\end{array}\right)\right| \leq C\left(1+y_{1}^{N}+y_{2}^{N}\right) e^{-2 \pi\left(y_{1}+y_{2}\right)} .
$$

We insert the estimate (12.4) into the left-hand side (LHS) of 12.1 and arrive at the inequality

and likewise

$$
\operatorname{LHS}(s, s) \leq C(2 \pi)^{-2 s} e^{-2 s}(s+N-1)^{2 s+2 N-3}
$$

$$
\operatorname{LHS}(s, 0) \leq C(2 \pi)^{-s} e^{-s}(s+N-1)^{s+N-3 / 2} .
$$

Conjecturally we could even take any $N>0$ (cf. Remark 11.15). In any case, if we compare (12.6) with (12.3) we see that the exponential decay for the Whittaker functional established in Theorem 11.13 is optimal. In fact, any better exponential decay rate would lead to decrease of $(2 \pi)^{-2 s}$ to $(2 \pi+a)^{-2 s}$ for some $a>0$ on the right of (12.6); this would contradict the asymptotics in (12.3).

12.2 Automorphic holomorphic triple products. We introduce a holomorphic version of triple products and raise some natural questions. The setting here is: $G$ a semisimple noncompact Lie group and $\Gamma<G$ a cocompact lattice. For three automorphic forms $\phi_{1}, \phi_{2}, \phi_{3}$ on $\Gamma \backslash G$ one forms the automorphic triple product, or automorphic trilinear functional in the terminology of J. Bernstein and A. Reznikov,

$$
\ell_{\text {aut }}\left(\phi_{1}, \phi_{2}, \phi_{3}\right)=\int_{\Gamma \backslash G} \phi_{1}(\Gamma g) \phi_{2}(\Gamma g) \phi_{3}(\Gamma g) d(\Gamma g) .
$$

Assume now that the $\phi_{i}$ are Maaß forms so that the integral defining $\ell_{\text {aut }}$ is effectively over the locally symmetric space $\Gamma \backslash X$. From the general 
theory we know that the $\phi_{i}$ extend to holomorphic functions $\tilde{\phi}_{i}$ on the local crown domain $\Gamma \backslash \Xi$. For the moment we restrict ourselves to the basic case of $G=\operatorname{Sl}(2, \mathbb{R})$ with comments on the general situation thereafter.

We form the holomorphic automorphic triple product by

$$
\ell_{\text {aut }}^{\text {hol }}\left(\phi_{1}, \phi_{2}, \phi_{3}\right)=\int_{\Gamma \backslash \Xi} \tilde{\phi}_{1}(\Gamma z) \tilde{\phi}_{2}(\Gamma z) \tilde{\phi}_{3}(\Gamma z) d(\Gamma z)
$$

where $d(\Gamma z)$ is the measure on $\Gamma \backslash \Xi$ induced from the Haar measure on $X_{\mathbb{C}}$. That $\ell_{\text {aut }}^{\text {hol }}$ is actually defined is the content of the next lemma.

Lemma 12.1. Let $G=\operatorname{Sl}(2, \mathbb{R})$ and $\Gamma<G$ be a cocompact lattice. Let $\phi_{1}, \phi_{2}, \phi_{3}$ be Maaß automorphic forms. Then the integral defining $\ell_{\text {aut }}^{\text {hol }}$ converges absolutely.

Proof. In view of [KrS1, Th. $51 \&$ Th. 6.17], there exists a constant $C>0$ such that we have for all $Y \in \Omega$

$$
\sup _{g \in G}\left|\tilde{\phi}_{i}\left(\Gamma g \exp (i \pi Y / 2) \cdot x_{0}\right)\right| \leq C|\log \cos \alpha(\pi Y / 2)| \text {. }
$$

Thus in view of the polar decomposition of the measure $\Xi$ (see $[\mathrm{KrS} 2$, Prop. 4.6]), we get

$$
\left|\ell_{\text {aut }}^{\text {hol }}\left(\phi_{1}, \phi_{2}, \phi_{3}\right)\right| \leq C^{3} \int_{0}^{1}|\log \cos \alpha(\pi Y / 2)|^{3} \cdot \sin \alpha(\pi Y) d Y<\infty,
$$

and this proves the lemma.

Problem 12.2. Determine the relation between $\ell_{\text {aut }}$ and $\ell_{\text {aut }}^{\text {hol }}$ and explain its significance.

REMARK 12.3. For a general semisimple Lie group the integrals defining $\ell_{\mathrm{aut}}^{\text {hol }}$ are not absolutely convergent. However, we have some freedom in the choice of the $G$-invariant measure on $\Xi$. Under the parameterization map $p: G / M \times \Omega^{+} \rightarrow \Xi$ the pull back of the Haar measure $d z$ on $\Xi$ is given by

$$
p^{*}(d z)=d(g M) \times J(Z) d Z
$$

with $J(Z)=\prod_{\alpha \in \Sigma^{+}}[\sin \alpha(\pi Z)]^{m_{\alpha}}$ (see $[\mathrm{KrS} 2$, Prop. 4.6]). For example if we replace $J$ by a sufficiently high power $J^{k}$, then $\ell_{\text {aut }}^{\text {hol }}$ is defined with regard to this measure. It is possible to carry out the details using the results obtained in this article.

12.3 Exponential decay of automorphic triple products. With the methods of analytic continuation one can prove exponential decay of automorphic triple products (see $[\mathrm{S}]$ and $[\mathrm{BeR}]$ for the first results). To be a more precise, consider a compact locally symmetric space $\Gamma \backslash X$ and fix a Maaß form $\phi$. Then for a Maaß form $\phi_{\pi}$ corresponding to an automorphic 
representation $\pi$ there is interest in finding the precise exponential decay of

$$
\left|\ell_{\text {aut }}\left(\phi, \phi_{\pi}, \overline{\phi_{\pi}}\right)\right|
$$

in terms of the parameter $\lambda(\pi)$ of $\pi$. This was first determined by Sarnak for $G=\mathrm{Sl}(2, \mathbb{C})[\mathrm{S}]$ and then by Petridis $[\mathrm{P}]$ and Bernstein-Reznikov $[\mathrm{BeR}]$ for $G=\operatorname{Sl}(2, \mathbb{R})$. Optimal bounds for all rank one groups were established in $[\mathrm{KrS1} 1$. For higher rank groups, $\operatorname{such}$ as $\mathrm{Sl}(n, \mathbb{R})$, partial results were obtained in [KrS1]. These bounds however fail to be optimal in general. The results in this paper combined with the methods of [BeR] and [KrS1] allow us to establish non-trivial (although) non-optimal bounds for the exponential decay of automorphic triple products.

\section{Appendix: Leading Exponents of Holomorphically Extended Elementary Spherical Functions}

In this appendix we prove Theorem 7.2 and the table of Theorem 7.9 for the asymptotic behavior of the norm of the holomorphic extension of the orbit map $G / K \ni g K \rightarrow \pi(g) v$ of a spherical vector $v \in \mathcal{H}$ in an irreducible spherical representation $(H, \pi)$ of $G$ when the argument approaches the distinguished boundary of the crown domain $\Xi$. The key property equation (7.1) translates this to the problem of finding the asymptotic behavior of certain solutions of a system of differential equations when approaching the singular locus of the system. In the theory of ordinary Fuchsian differential equations this boils down to the study of characteristic exponents at its singular points and their relation to the monodromy of the system. A beautiful application (closely related to our problem in fact, via (5.9)) of this classical theory is the study of the asymptotic behavior of certain classes of oscillatory integrals via the monodromy of the Gauß-Manin connection of the Milnor fibration of the phase function [Ma].

In the case of "Fuchsian systems" of differential equations in several complex variables we first need to develop some fundamental facts on exponents and their first properties. In the case of a regular holonomic $\mathcal{D}$ module of the form $\mathcal{D} / \mathcal{J}$ on $\mathbb{C}^{n}$ which is $\mathcal{O}$-coherent on the complement of a hyperplane arrangement in $\mathbb{C}^{n}$ we propose a definition of the set of exponents of local solutions of the system of equations $D \phi=0, \forall D \in \mathcal{J}$ at any point $\eta \in \mathbb{C}^{n}$. This translates our original problem to that of determining the set of exponents of a special solution to Harish-Chandra's radial system of differential equations on $A_{C} \cdot x_{0}$ (namely the holomorphic 
extension of the restriction of the elementary spherical function to $A . x_{0}$ ) at the extremal boundary points $t(\eta)^{2} x_{0}$ of $T_{\Omega}$.

What turns this into a successful method is the fact that there exists a well-behaved parameter deformation of Harish-Chandra's radial system of differential equations for which we have rather explicit knowledge of the monodromy representation of its solutions for generic parameters. This deformation is the hypergeometric system of differential equations $[\mathrm{HeO}]$, [HeS], [O5]. Its monodromy factors through an affine Hecke algebra, thus bringing the representation theory of affine Hecke algebras into play. In the spirit of the study of the Bessel function equations [O3] this leads to the description of the set of all exponents of the hypergeometric system at $t(\eta)^{2} \cdot x_{0}$. Using that and the relation between exponents and monodromy (which we will carefully establish below) we can compute the leading exponents of the holomorphically extended hypergeometric function at the points $t(\eta)^{2} x_{0}$.

Specialization of the parameters then leads to the desired lower bounds for the leading exponents of holomorphically extended elementary spherical functions on a Riemannian symmetric space $X$, leading to the proof of Theorem 7.2 and Theorem 7.9.

One remarkable phenomenon that comes out of these considerations is that the leading exponent of the hypergeometric function at an extremal point $t(\eta)^{2} \cdot x_{0}$ is related to a leading character $\sigma_{\eta}$ of the isotropy group $W_{\eta}^{a} \subset W$ of $t(\eta)^{2} \cdot x_{0}$ which depends only on the geometry of $\Omega$ locally at the extremal point $\eta$, but not on the multiplicity $m$ (if $m$ is real and satisfies certain inequalities which hold for the multiplicity functions of Riemannian symmetric spaces).

13.1 Exponents and Hyperplane Arrangements. In this subsection we propose a definition of exponents of local Nilsson class functions $[\mathrm{Bj}$, Ch. 6.4] on the complement of a hyperplane arrangement of $\mathbb{C}^{n}$ at points $\eta \in \mathbb{C}^{n}$. The main results are the invariance of the exponents at $\eta$ for local monodromy at $\eta$ and the relation between exponents and monodromy.

Let $\eta \in \mathbb{C}^{n}$ and let $\phi$ be a local Nilsson class function at $\eta$. By this we mean a multivalued holomorphic function $\phi$ on the complement $N \backslash Y:=$ $N^{\text {reg }}$ of an analytic hypersurface $Y \subset \mathbb{C}^{n}$ inside a small open ball $N \subset \mathbb{C}^{n}$ centered at $\eta$ such that

LN1: $\phi$ has finite determination order in $N^{\text {reg }}$.

LN2: The pull back of any branch of $\phi$ via any holomorphic map $j: \mathbb{D} \rightarrow N$ with the property that $j^{-1}(Y) \subset\{0\}$ has moderate growth at $0 \in \mathbb{D}$. 
Suppose that $j$ as in LN2 is an embedding such that $j(0)=\eta$. Then the pull back of (any branch of) $\phi$ via $j$ has a singular expansion at $\epsilon=0$ (where $\epsilon$ denotes the standard coordinate in the unit disk $\mathbb{D}$ ) of the form

$$
\phi(j(\epsilon))=\sum_{s, l} \epsilon^{s} \log ^{l}(\epsilon) f_{s, l}(\epsilon),
$$

a sum over a finite set of pairs $(s, l)$ with $s \in \mathbb{C}$ and $l \in \mathbb{Z}_{\geq 0}$, such that for each pair $(s, l)$ in this sum the function $f_{s, l}(\epsilon)$ is holomorphic on $\mathbb{D}^{\times}$with at most a pole at $\epsilon=0$. This expansion is obviously not unique, and even if one tries to make it unique by imposing additional requirements one will find that the set $S$ which enters in (13.1) will in general depend on the chosen embedding $j$ and of the chosen branch of $\phi$ (with respect to local monodromy in $N^{\text {reg }}$ ) in an essential way. In order to define exponents of $\phi$ at $\eta$ we assume from now on the following.

AR: For sufficiently small $N$ we may take $Y=Y^{\eta}$ to be a linear hyperplane arrangement centered at $\eta$.

We call a holomorphic map $i: \mathbb{D}^{n} \rightarrow N$ a standard coordinate map if

(i) $i(0, z)=\eta$ for all $z \in \mathbb{D}^{n-1}$;

(ii) $i\left(\mathbb{D}^{\times} \times \mathbb{D}^{n-1}\right) \subset N^{\text {reg; }}$

(iii) The lift of the map $i: \mathbb{D}^{\times} \times \mathbb{D}^{n-1} \rightarrow N^{\text {reg }}$ to the blow-up $X_{\eta} \rightarrow \mathbb{C}^{n}$ of $N$ at the point $\eta$ extends to a coordinate map $i: \mathbb{D}^{n} \rightarrow X_{\eta}$ such that $i\left(\mathbb{D}^{n}\right) \cap Z=\emptyset$, where $Z$ denotes the strict transform of $Y \cap N$.

Let $i$ be a standard coordinate map. Choose a base point $p=i(P) \in$ $i\left(\mathbb{D}^{\times} \times \mathbb{D}^{n-1}\right)$ and fix a germ $\phi_{p}$ of a branch of $\phi$ at $p$. Let $C \subset \mathbb{D}^{\times}$ be a cut disk (the complement in $\mathbb{D}$ of a ray emerging from 0 ) such that $P \in C \times \mathbb{D}^{n-1}$. The pull back of $\phi_{p}$ via $i$ to $P \in C \times \mathbb{D}^{n-1}$ is the germ of a Nilsson class function on $\mathbb{D}^{\times} \times \mathbb{D}^{n-1}$. Hence we have the following standard result $[\mathrm{Bj}$, Prop. 4.4.2]:

Proposition 13.1. There exists a finite set $S$ of pairs $(s, l)$ with $s \in \mathbb{C}$ and $l \in \mathbb{Z}_{\geq 0}$ such that the unique analytic continuation of $i^{*}\left(\phi_{p}\right)$ to $C \times \mathbb{D}^{n-1}$ admits an expansion of the form

$$
\phi_{p}\left(i\left(\epsilon, z^{\prime}\right)\right)=\sum_{(s, l) \in S} \epsilon^{s} \log ^{l}(\epsilon) f_{s, l}\left(\epsilon, z^{\prime}\right),
$$

where each $f_{s, l}$ extends meromorphically to $\mathbb{D}^{\times} \times \mathbb{D}^{n-1}$.

As before this expansion is not unique for obvious reasons but we can rearrange (13.2) in such a way that

(a) All $f_{s, l}$ extend holomorphically on $\mathbb{D}^{n}$; 
(b) If the pairs $(s, l)$ and $\left(s^{\prime}, l^{\prime}\right)$ occur in (13.2) then $s-s^{\prime}$ is not equal to a nonzero integer; and

(c) If the pair $(s, l)$ occurs in (13.2) then there exists an $l^{\prime} \in \mathbb{Z}_{\geq 0}$ such that $f_{s, l^{\prime}}(0, \cdot) \not \equiv 0$.

That makes the expansion unique.

DeFinition 13.2. Let $\phi$ be a local Nilsson class function at $\eta \in \mathbb{C}^{n}$ and let $i: \mathbb{D}^{\times} \times \mathbb{D}^{n-1} \rightarrow N^{\text {reg }}$ be a standard coordinate map. Choose a base point $p$ in the image of $i$, and choose a germ $\phi_{p}$ of a branch of $\phi$ at $p$. We define the finite set $E^{\eta, i, p}\left(\phi_{p}\right) \subset \mathbb{C} \cup\{\infty\}$ of exponents of $\phi_{p}$ at $\eta$ as the projection of the finite set $S \subset \mathbb{C} \times \mathbb{Z}_{>0}$ defined above to the first component if $\phi \neq 0$. We put $E^{\eta, i, p}(0)=\{\infty\}$.

Proposition 13.3. The set $E^{\eta, i, p}\left(\phi_{p}\right)$ is independent of the choice of $i$ (satisfying the requirements (i),(ii) and (iii) above) and is independent of analytic continuation of $\phi_{p}$ within $N^{\mathrm{reg}}$. Hence we may speak about the set of exponents $E^{\eta}(\phi)$ without referring to a specific branch of $\phi$ and coordinate map $i$. If $w: N \rightarrow N$ is a linear automorphism of the hyperplane arrangement $Y^{\eta}$ then $E^{\eta}\left(\phi^{w}\right)=E^{\eta}(\phi)$.

Proof. By equation (13.2) it is clear that $E^{\eta, i, p}\left(\phi_{p}\right)$ is independent of analytic continuation of $\phi_{p}$ along paths inside $i\left(\mathbb{D}^{\times} \times \mathbb{D}^{n-1}\right)$. Suppose that $i, i^{\prime}$ both satisfy the requirements above, and suppose that $i\left(\{0\} \times \mathbb{D}^{n-1}\right) \cap$ $i^{\prime}\left(\{0\} \times \mathbb{D}^{n-1}\right) \neq \emptyset$. Let $V \subset i\left(\{0\} \times \mathbb{D}^{n-1}\right) \cap i^{\prime}\left(\{0\} \times \mathbb{D}^{n-1}\right) \subset E$ be a connected contractible open set (where $E$ denotes the exceptional divisor). By the properties of $i$ and $i^{\prime}$ we have $\left(i^{\prime}\right)^{-1}\left(i\left(\epsilon, z^{\prime}\right)\right)=\left(\epsilon^{\prime}, w^{\prime}\right)$ with $\epsilon^{\prime}\left(\epsilon, z^{\prime}\right)=\epsilon h\left(\epsilon, z^{\prime}\right)$ where $h$ is holomorphic and nonzero on $i^{-1}(V)$. If we plug this in the expansion (13.2) we see that the exponents defined by $i$ and by $i^{\prime}$ are equal if we use branches of $\phi$ on the image of $i$ and on the image of $i^{\prime}$ which are related by analytic continuation via the connected component of the intersection of the images of $i$ and $i^{\prime}$ which contains $V$. By AR we see that that any path in $N^{\text {reg }}$ is homotopic to a path which is contained in a finite union of coordinate patches of the form $i\left(\mathbb{D}^{\times} \times \mathbb{D}^{n-1}\right)$. With the above this shows at once that the set of exponents does not depend on the choice of the coordinate map $i$ and is independent for analytic continuation of $\phi_{p}$ within $N^{\text {reg }}$. For the last assertion we remark that $i_{w}=w \circ i$ is also a standard coordinate map, hence $E^{\eta}\left(\phi^{w}\right)=E^{\eta, i_{w}, w(p)}\left(\left(\phi^{w}\right)^{w(p)}\right)=E^{\eta, i, p}\left(\phi_{p}\right)=E^{\eta}(\phi)$.

What lies behind this notion of exponents is the well-known "decone construction" on a central hyperplane arrangement. This elementary 
construction implies that if $Y^{\eta}$ is nonempty then $N^{\text {reg }}$ is a isomorphic to a product

$$
N^{\mathrm{reg}} \simeq \mathbb{D}^{\times} \times E^{\mathrm{reg}}
$$

Indeed, the restriction of the Hopf fibration $p: \mathbb{C}^{n} \backslash\{0\} \rightarrow E=\mathbb{P}\left(\mathbb{C}^{n}\right)$ to the complement of one of the hyperplanes $H$ of $Y$ is a trivial fibration since $E \backslash \mathbb{P}(H) \simeq \mathbb{C}^{n-1}$ is contractible. Hence the further restriction of this fibration to $N^{\text {reg }}$ is a fortiori trivial. Thus we have a decomposition

$$
\pi_{1}\left(N^{\mathrm{reg}}, p\right) \simeq \mathbb{Z} \times \pi_{1}\left(E^{\mathrm{reg}},[p]\right) .
$$

Now let $\mathcal{L} \subset \mathcal{O}\left(N^{\text {reg }}\right)$ be a local system of finite rank $r$ of germs of Nilsson class function on $N^{\text {reg. }}$. We remark that, as a result of LN1, the germs of any local Nilsson class function $\phi$ on $N^{\text {reg }}$ are contained in such a local system.

DeFinition 13.4. We denote by $T_{p}^{\eta}$ the monodromy map on $\mathcal{L}_{p} \simeq \mathbb{C}^{r}$ which corresponds to analytic continuation along the loop $\gamma_{p}^{\eta}: t \rightarrow$ $\exp (2 i \pi t) p$. Observe that $\left[\gamma_{p}^{\eta}\right]$ is a generator of $\mathbb{Z}$ in (13.4).

In view of (13.4) we may use $T_{p}^{\eta}$ to split the sheaf $\mathcal{L}$ as a direct sum

$$
\mathcal{L}=\bigoplus_{t \in \mathbb{C}^{\times}} \mathcal{L}^{\eta}(t)
$$

of generalized eigensheaves of $T^{\eta}$. In view of (13.2) it is clear that if $\phi \neq 0$ then $\phi \in \mathcal{L}_{p}^{\eta}(t)$ iff $E^{\eta}(\phi)=\{s\}$ for some exponent $s \in \mathbb{C}$ such that $t=$ $\exp (2 i \pi s)$.

Given $s \in \mathbb{C} \cup\{\infty\}$ we define a subsheaf $F_{s}^{\eta}(\mathcal{L}) \subset \mathcal{L}^{\eta}(\exp (2 i \pi s))$ of $\mathcal{L}$ by setting for each $p \in N^{\text {reg: }}$

$$
F_{s}^{\eta}(\mathcal{L})_{p}=\left\{\phi \in \mathcal{L}_{p} \mid E^{\eta}(\phi)=\{\kappa\} \text { with } \kappa-s \in \mathbb{Z}_{\geq 0} \cup\{\infty\}\right\} .
$$

One checks easily that this is a linear subspace of $\mathcal{L}_{p}$. By Proposition 13.3 it is invariant for the parallel transport in the local system $\mathcal{L}$, hence it defines a subsheaf. Moreover, these subsheaves of $\mathcal{L}$ are invariant for the action of the group of automorphisms of $\mathcal{L}$ which are induced by linear automorphisms of the arrangement $Y^{\eta}$. For each $t \in \mathbb{C}^{\times}$the subsheaves $F_{s}^{\eta}(\mathcal{L}) \subset \mathcal{L}$ with $s \in \mathbb{C}$ such that $\exp (2 i \pi s)=t$ define a descending filtration

$$
\cdots \supset F_{s}^{\eta}(\mathcal{L}) \supset F_{s+1}^{\eta}(\mathcal{L}) \supset \ldots
$$

of the direct summand $\mathcal{L}^{\eta}(t)$ of $\mathcal{L}$.

Definition 13.5. We define a local system $\operatorname{Gr}^{\eta}(\mathcal{L})$ by

$$
\operatorname{Gr}^{\eta}(\mathcal{L})=\bigoplus_{s \in \mathbb{C}} \operatorname{Gr}_{s}^{\eta}(\mathcal{L}), \text { with } \operatorname{Gr}_{s}^{\eta}(\mathcal{L})=F_{s}^{\eta}(\mathcal{L}) / F_{s+1}^{\eta}(\mathcal{L})
$$


For each $s \in \mathbb{C}$ we define the multiplicity $\operatorname{mult}^{\eta}(\mathcal{L}, s)$ of $s$ as an exponent at $\eta$ of the system of $\mathcal{L}$ by

$$
\operatorname{mult}{ }^{\eta}(\mathcal{L}, s)=\operatorname{dim}\left(\operatorname{Gr}_{s}^{\eta}(\mathcal{L})\right) .
$$

Definition 13.6. The (multi-)set $E^{\eta} \subset \mathbb{C}$ of exponents of $\mathcal{L}$ at $\eta$ are the complex numbers $s \in \mathbb{C}$ such that $\operatorname{mult}^{\eta}(\mathcal{L}, s)>0$.

Corollary 13.7. The (multi-) set $\exp \left(2 i \pi E^{\eta}\right) \subset \mathbb{C}^{\times}$is the generalized eigenvalue spectrum of $T^{\eta}$ acting on $\mathcal{L}$.

ExAmple 13.8. Consider for $\mu \in \mathfrak{a}_{\mathbb{C}}{ }^{*}$ the sheaf $\mathcal{L}$ of local solutions of the set of equations

$$
\partial(p) \phi=p(\mu) \phi, \forall p \in \mathbb{C}\left[\mathfrak{a}_{\mathbb{C}}{ }^{*}\right]^{W},
$$

and let $\eta \in \mathfrak{a}_{\mathbb{C}}$ be any point. Any local solution $\phi$ is holomorphic at $\eta$ and is completely determined by its harmonic derivatives $\partial(q)(\phi)(\eta)$ at $\eta$. Hence the set of exponents of $\mathcal{L}$ at $\eta$ is independent of $\eta$ and $\mu$, and is equal to the set $0,1, \ldots,\left|\Sigma_{+}^{l}\right|$ where $\operatorname{mult}^{\eta}(\mathcal{L}, s)=\operatorname{dim} \operatorname{Harm}_{s}(W)$, the dimension of the space of $W$-harmonic polynomials of homogeneous degree $s$.

Example 13.9. Consider the sheaf $\mathcal{L}$ of local solutions of (7.12). Suppose that $\eta \in \mathfrak{a}_{\mathbb{C}}$ reg is a regular point. Again a local solution $\phi$ of (7.12) near $\eta$ is holomorphic at $\eta$ and is completely determined by its harmonic derivatives $\partial(q)(\phi)(\eta)$ at $\eta$. Hence the answer is the same as in the previous example.

ExAmPle 13.10. Let $\mathcal{L}$ be as in the previous example, but now we take $\eta=i \pi \omega_{j} / k_{j}$ as in subsection 2.2. The exponents of $\mathcal{L}$ at $\eta$ are equal to $-\left|\Sigma_{\eta,+}^{a}\right|, \ldots,\left|\Sigma_{+}^{l}\right|-\left|\Sigma_{\eta,+}^{a}\right|$, and if $\phi_{\mu}$ denotes the holomorphic extension to $\mathfrak{a}+i \pi \Omega$ of the spherical function, then for generic $\mu$ we have $E^{\eta}\left(\phi_{\mu}\right)=$ $\left\{\left|\Sigma_{\eta,+}\right|-\left|\Sigma_{\eta,+}^{a}\right|\right\}$ (see Proposition 7.8).

\subsection{Harish-Chandra's radial system of differential equations.}

In this subsection we describe the system of differential equations we are mainly interested in, the radial differential equations for elementary spherical functions $\phi_{\mu}^{X}$ on a Riemannian symmetric space $X=G / K$ restricted to a maximal flat, totally geodesic subspace $A_{X}=A . x_{0} \subset X$.

The elementary spherical function $\phi_{\mu}^{X}$ (with $\mu \in \mathfrak{a}_{\mathbb{C}}{ }^{*}$ ) on $X=G / K$ is a $K$-invariant solution of the $G$-invariant system of differential equations

$$
\left(\Delta-\gamma_{X}(\Delta)(\mu)\right) \phi=0 \forall \Delta \in \mathbf{D}(X)
$$

where $\mathbf{D}(X)$ denotes the ring of $G$-invariant differential operators on $X$, and where $\gamma_{X}: \mathbf{D}(X) \rightarrow \mathbb{C}\left[\mathfrak{a}^{*}\right]^{W}$ is the Harish-Chandra isomorphism.

By separation of variables we see that the restriction of $\phi_{\mu}^{X}$ to $A_{X}$ is a $W$-invariant solution of the system of differential equations

$$
\left(D-\gamma_{X}(D)(\mu)\right) \phi=0 \forall D \in \mathcal{R}_{X}
$$


on $A_{X}$ or on its complexification $A_{X, \mathbb{C}}=A_{\mathbb{C}} \cdot x_{0}$, where $\mathcal{R}_{X} \simeq \mathbf{D}(X)$ is the algebra of radial parts of the operators $\Delta \in \mathbf{D}(X)$. Notice that we use the same notation $\gamma_{X}$ for the Harish-Chandra isomorphism defined on $\mathcal{R}_{X}$.

Let $T_{X}=T \cdot x_{0} \subset A_{X, \mathbb{C}}$ be the compact form of $A_{X, \mathbb{C}}$. It is a maximal flat totally geodesic subspace of a compact dual symmetric space $U / K$ (which is by our choices simply connected). The restrictions to $T_{X}$ of the zonal spherical functions of $U / K$ are $W$-invariant simultaneous eigenfunctions of $\mathcal{R}_{X}$. Since these zonal polynomials constitute a linear basis of the space of $W$-invariant Laurent polynomials on $A_{X, \mathbb{C}}=A_{\mathbb{C}} / F$ this implies that the operators in $\mathcal{R}_{X}$ descend to polynomial differential operators on the complex affine quotient space $W \backslash A_{\mathbb{C}} / F$.

13.3 The hypergeometric system of differential equations. In this subsection we describe a parameter deformation of the Harish-Chandra system (13.12) of differential equations that we will use to study properties of solutions of (13.12). This parameter family of systems of differential equations is called the system of hypergeometric equations associated with root systems. As was explained, this deformation is an essential ingredient for the computation of the leading exponents (7.2) of the spherical functions at extremal points of $T_{\Omega}$.

We need to introduce some notation. Let $\Sigma$ be a (not necessarily reduced) irreducible root system in $\mathfrak{a}^{*}$. We consider indeterminates $\mathbf{m}_{\alpha}$ which are labeled by the $W$-orbits of the roots $\alpha \in \Sigma$ (in other words, $\mathbf{m}_{\alpha}=\mathbf{m}_{\beta}$ if $W \alpha=W \beta$ ). Let $\mathbb{C}\left[\mathbf{m}_{\alpha}\right]$ be the complex polynomial algebra over these indeterminates $\mathbf{m}_{\alpha}$. If $X$ is a Riemannian symmetric space with restricted root system isomorphic to $\Sigma$ then $m_{\alpha}^{X} \in \mathbb{N}$ denote the root multiplicities of $X$.

The following result is one of the cornerstones of the theory of hypergeometric functions for root systems.

Theorem 13.11 [O1], [He]. Let $\mathbb{A}$ denote the Weyl algebra of polynomial differential operators on the complex affine space $W \backslash A_{\mathbb{C}} / F \simeq \mathbb{C}^{n}$ with coefficients in the polynomial ring $\mathbb{C}\left[\mathbf{m}_{\alpha}\right]$. There exists a unique subalgebra $\mathcal{R} \subset \mathbb{C}\left[\mathbf{m}_{\alpha}\right] \otimes \mathbb{A}$ with the following properties:

(1) The algebra $\mathcal{R}$ is isomorphic to the polynomial ring $\mathbb{C}\left[\mathbf{m}_{\alpha}\right]\left[\mathfrak{a}_{\mathbb{C}}^{*}\right]^{W}$ via a Harish-Chandra isomorphism $\gamma$ of algebras. This isomorphism $\gamma$ has the characterizing property that any element $D \in \mathcal{R}$ is asymptotically equal to the constant coefficient operator $\gamma(D)(\cdot-\rho(\mathbf{m}))$ on $A_{\mathbb{C}}$ (viewed as an element of the symmetric algebra on $\mathfrak{a}_{\mathbb{C}}$ ) along regular directions towards infinity in $A_{+}$. 
(2) If we specialize $\mathbf{m}$ at the multiplicity function $m^{X}$ for a Riemannian symmetric space $X=G / K$ with restricted root system $\Sigma_{X} \subset \mathfrak{a}^{*}$ such that $\Sigma^{l}=\Sigma_{X}^{l}$, such that $A_{\mathbb{C}}$ is the maximal torus of $G_{\mathbb{C}}$, then $\mathcal{R}$ specializes to $\mathcal{R}_{X}$ and $\gamma$ to the Harish-Chandra isomorphism $\gamma_{X}$.

It is remarkable that the theory of Dunkl operators provides a proof of this theorem which is both elementary and simple [He].

Proposition 13.12 [HeO],[O5, Rem.6.10]. Let $\mathbb{D}^{\text {reg }}$ be the ring of algebraic differential operators on the affine variety $A_{\mathbb{C}}^{\text {reg }} / F$. For each multiplicity parameter $m=\left(m_{\alpha}\right)$ and $\mu \in \mathfrak{a}_{\mathbb{C}}^{*}$ let $\mathcal{I}_{m, \mu} \subset \mathbb{D}^{\text {reg }}$ denote the $W$-invariant left ideal

$$
\mathcal{I}_{m, \mu}:=\sum_{D \in \mathcal{R}_{m}} \mathbb{D}^{\mathrm{reg}}\left(D-\gamma_{m}(D)(\mu)\right) .
$$

Here $\mathcal{R}_{m}$ is the specialization of $\mathcal{R}$ at $m$, and $\gamma_{m}$ the corresponding HarishChandra homomorphism.

Consider the $\mathbb{D}^{\text {reg }}$-module $\mathcal{M}_{\mu, m}=\mathbb{D}^{\text {reg }} / \mathcal{I}_{m, \mu}$ on $A_{\mathbb{C}}{ }^{\text {reg }} / F$. Then in the terminology of Chapter IV, section 7 of [Bo et al], $\mathcal{M}_{\mu, m}$ is an algebraic connection on $A_{\mathbb{C}}{ }^{\mathrm{reg}} / F=A_{\mathbb{C}} / F-\{\delta=0\}$ of rank $|W|$ which is regular. Moreover $\mathcal{M}_{\mu, m}$ is $W$-equivariant.

Proof. The elements of $\mathcal{R}_{m}$ are algebraic and the coefficients are known to be regular on $A_{\mathbb{C}}^{\text {reg }} / F$ (the simplest way to see this is to use DunklCherednik operators [He], [O4]). It is known that $\mathcal{M}_{\mu, m}$ is $\mathcal{O}\left(A_{\mathbb{C}}{ }^{\text {reg }} / F\right)$ free of rank $|W|$ by $[\mathrm{HeO}]$, and it is clear that $\mathcal{M}_{\mu, m}$ is $W$-equivariant. It remains to prove the regularity.

The elements of $\mathcal{R}_{m}$ descend to the regular part of the adjoint torus $A_{\mathbb{C}}{ }^{\text {adj,reg }} / F$ with character lattice $Q=\mathbb{Z} \Sigma \subset \mathfrak{a}^{*}$. We view this as an open subset of the toric completion of $A_{\mathbb{C}}$ adj $/ F$ associated with the decomposition of $\mathfrak{a}^{*}$ in Weyl chambers. This is a projective variety. It clearly suffices to prove the regularity on $A_{\mathbb{C}}{ }^{\text {adj,reg }} / F$.

On $A_{\mathbb{C}}{ }^{\text {adj,reg }} / F$ one can explicitly rewrite the module $\mathcal{M}_{\mu, m}$ as a connection of rank $|W|$ with logarithmic singularities at infinity (see $[\mathrm{HeO}]$ ). According to $[\mathrm{HeO}]$ the connection matrix depends polynomially on the parameters $\mu$ and $m$. It remains to show that the connection is also regular singular at the components of the discriminant locus $\delta=0$. Since the connection depends polynomially on the parameters $\mu, m$ it is easy to see that the set of parameters $\mu, m$ for which the connection is regular singular is a Zariski-closed set. If $m=0$ the system is trivially regular singular. If $\mu$ is sufficiently generic then the theory of shift operators gives equivalences between the modules $\mathcal{M}_{\mu, m}$ and $\mathcal{M}_{\mu, m^{\prime}}$ if $m-m^{\prime}$ belong to the "lattice of 
integral shifts" (see, e.g. [O1] or [HeS]) in the space of multiplicity parameters. The result follows.

Remark 13.13. The element $u=1 \in \mathcal{M}_{\mu, m}$ is a cyclic vector. Via $u$ the complex vector space of $D$-module homomorphisms of $\mathcal{M}_{\mu, m}$ to $\mathcal{O}_{p}$ correspond to the space $\mathcal{L}_{p}(\mu, m)$ of solutions in $\mathcal{O}_{p}$ of the $W$-invariant system of differential equations

$$
\left(D-\gamma_{m}(D)(\mu)\right) \phi=0 \quad \forall D \in \mathcal{R}_{m}
$$

on $A_{\mathbb{C}}^{\text {reg }} / F$.

Corollary 13.14. The local system $\mathcal{L}(\mu, m)$ of germs of solutions of (13.14) is a local system of germs of Nilsson class functions on $\mathfrak{a}_{\mathbb{C}}{ }^{\text {reg. }}$. Hence the results of subsection 13.1 are applicable to $\mathcal{L}(\mu, m)$.

Proof. By [Bj, Prop.4.6.6] it is sufficient to check the moderate growth conditions for solutions of (13.14) on the dense open set of subregular points of $\delta=0$. Since we can rewrite the system (13.14) as a meromorphic connection on $\mathfrak{a}_{\mathbb{C}}{ }^{\text {reg }}$ which is regular singular along $\delta=0$ according to Proposition 13.12 this follows from [Bo et al, Rem. (5.9)] (see also [D1]).

Let $X$ be a Riemannian symmetric space with maximal flat geodesic subspace $A . x_{0}$. The holomorphic extension of the restriction to $A . x_{0}$ of the spherical function $\phi_{\mu}^{X}$ to $A T_{\Omega}^{2} x_{0}$ is a holomorphic $W$-invariant solution of (13.12) on $A T_{\Omega}^{2} \cdot x_{0}$. This function is the specialization of a holomorphic family (in the parameter $m$ ) of solutions of (13.14) by virtue of the following theorem:

Theorem $13.15([\mathrm{HeO}],[\mathrm{HeS}],[\mathrm{O} 5])$. There exists an $\epsilon>0$ such that for all multiplicity parameters $m \in \mathcal{Q}(-\epsilon)$, the space of multiplicity parameters such that $\operatorname{Re}\left(m_{\alpha}\right) \geq-\epsilon \forall \alpha \in \Sigma$, the hypergeometric system (13.14) has a unique solution $\phi_{\mu, m}$, the hypergeometric function, which extends to a $W$ invariant and holomorphic function on $A T_{\Omega}^{2} \cdot x_{0}$. The function $(t, \mu, m) \rightarrow$ $\phi_{\mu, m}(t)$ is holomorphic on $\left(A T_{\Omega}^{2} \cdot x_{0}\right) \times \mathcal{Q}(-\epsilon) \times \mathfrak{a}_{\mathbb{C}}{ }^{*}$.

Recall the covering map $\pi: \mathfrak{a}_{\mathbb{C}} \rightarrow A_{\mathbb{C}} / F \simeq A_{\mathbb{C}} \cdot x_{0}$ of (7.15) which is given by the exponential map $\pi(X)=\exp (\pi i X) F$. Via this map we will lift the differential equations (13.14) to $\mathfrak{a}_{\mathbb{C}}{ }^{\text {reg }}$ and work on $\mathfrak{a}_{\mathbb{C}}$ rather than $A_{\mathbb{C}} / F$. On this space the system of differential equations (13.14) is invariant for the action of the affine Weyl group $W^{a}=W \ltimes Q^{\vee}$. In particular, we will work on the tube domain $i \mathfrak{a}+\Omega \subset \mathfrak{a}_{\mathbb{C}}$ instead of $A T_{\Omega}^{2} / F \subset A_{\mathbb{C}} / F$ (recall that the logarithm is well defined on $A T_{\Omega}^{2}$ ). It is well known $[\mathrm{HeO}]$ that the spherical system of eigenfunction equations can be cast in the form of an 
integrable connection on $\mathfrak{a}_{\mathbb{C}}$ with singularities along the collection of affine hyperplanes $\alpha(H) \in \mathbb{Z}$ (not $\in \pi i \mathbb{Z}$ as in [HeO], since we have multiplied everything by $\left.(\pi i)^{-1}\right)$.

13.4 The indicial equation. We will show in this subsection that the exponents of the hypergeometric equations (13.14) at $\eta \in \mathfrak{a}_{\mathbb{C}}$ coincide with the eigenvalues of the residue matrix of a specially chosen integrable connection with simple poles which is equivalent to (13.14). The characteristic equation of the residue matrix has coefficients which are polynomials in the parameters $m_{\alpha}$. This equation is called the indicial equation of (13.14) at $\eta$.

Let us first construct an explicit standard coordinate map $i$ as used in the definition of the set of exponents. Consider a parameterized line $x \rightarrow \eta+x V_{1}$ through $\eta$, where $V_{1}$ is small and chosen in such a way that this line is not contained in the union of the singular affine hyperplanes. We choose coordinates $\left(z_{1}=\epsilon, z_{2}, \ldots z_{n}\right)$ (with $z_{1}=\epsilon \in \mathbb{D}^{\times}$and for $i>1$ : $\left.z_{i} \in \mathbb{D}\right)$, which we will often write as $z=\left(\epsilon, z^{\prime}\right) \in \mathbb{D}^{\times} \times \mathbb{D}^{n-1}$ with $z^{\prime}=$ $\left(z_{2}, \ldots, z_{n}\right)$. First we choose $V_{2}, \ldots, V_{n}$ in $\mathfrak{a}$ such that $\left\|V_{i}\right\|$ is small for all $i$, and such that $\left(V_{1}, V_{2}, \ldots, V_{n}\right)$ is a basis of the real vector space $\mathfrak{a}$. Then our coordinate map $i$ is given by

$$
i\left(\epsilon, z^{\prime}\right)=\eta+\epsilon\left(V_{1}+\sum_{i \geq 2} z_{i} V_{i}\right) \in \mathfrak{a} .
$$

If we lift this coordinate map to the blow-up of $\mathfrak{a}_{\mathbb{C}}$ at $\eta$ then the coordinates can be naturally extended to the polydisc $\mathbb{D}^{n}$, and this is then a coordinate neighborhood of a regular point of the exceptional divisor $E$. The intersection of this neighborhood with $E$ is described by the equation $z_{1}=0$. The complement of $z_{1}=0$ in $\mathbb{D}^{n}$ is $\mathbb{D}^{\times} \times \mathbb{D}^{n-1}$, the "punctured polydisc". The Euler vector field $\mathcal{E}^{\eta}$ is given in these coordinates by $z_{1} \partial / \partial z_{1}=\epsilon \partial / \partial \epsilon$.

Let $p$ be a point in the punctured polydisc $\mathbb{D}^{\times} \times \mathbb{D}^{n-1}$ and let $\mathcal{O}_{p}$ denote the ring of holomorphic germs at $p$. Consider a subspace $U^{*}$ of dimension $|W|$ of the ring of holomorphic linear partial differential operators on $\mathbb{D}^{\times} \times \mathbb{D}^{n-1}$ such that at all points $p \in \mathbb{D}^{\times} \times \mathbb{D}^{n-1}$, the free $\mathcal{O}_{p^{-} \text {-module }}$ $\mathcal{O}_{p} \otimes U^{*}$ is a complement for the left ideal $\mathcal{I}_{\mu, m}$. We require further that the elements of $U^{*}$ commute with the Euler vector field $\mathcal{E}^{\eta}$ (in other words, they are homogeneous of degree 0 ), and that $1 \in U^{*}$. Such linear subspaces $U^{*}$ exist, for instance one could take as a basis $b_{i}=\epsilon^{\operatorname{deg}\left(q_{i}\right)} \partial\left(q_{i}\right)$, where $q_{i}$ runs over a homogeneous basis of $W$-harmonic polynomials on $\mathfrak{a}_{\mathbb{C}}^{*}$ with $q_{1}=1$.

We rewrite the differential equations (13.14) (with $\mu \in \mathfrak{a}_{\mathbb{C}}{ }^{*}$ ) in connection form with respect to the above basis $\left\{b_{i}\right\}$ and coordinates $\left\{z_{i}\right\}$. We 
define matrices $A_{\mu, m}^{i} \in \operatorname{End}_{\mathcal{O}_{p}}\left(\mathcal{O}_{p} \otimes U\right)$ (where $U$ denotes the dual of $U^{*}$, with dual basis $b_{i}^{*}$ ) which are characterized by the requirement that

$$
\frac{\partial}{\partial z_{i}} \circ b_{k} \in \sum_{j}\left(A_{\mu, m}^{i}\right)_{j k}^{\mathrm{tr}} b_{j}+\mathcal{I}_{\mu, m} .
$$

As an $\mathcal{O}_{p}$-module, the cyclic $D$-module $\left(M_{\mu, m}, u\right)$ is equal to $\mathcal{O}_{p} \otimes U^{*} u$, with basis $\overline{b_{i}}=b_{i} . u$. Then the desired (flat) connection form of (13.14) is defined on the free $\mathcal{O}_{p}$-module $\mathcal{O}_{p} \otimes U$ by

$$
\frac{\partial \Phi}{\partial z_{i}}=A_{\mu, m}^{i} \Phi \quad\left(\Phi \in \mathcal{O}_{p} \otimes U\right)
$$

By construction, if $\phi$ is a solution of (13.14) then

$$
\Phi(\phi):=\sum_{i} b_{i}(\phi) b_{i}^{*}
$$

is a solution vector of (13.17). Conversely, if $\Phi$ is a solution vector of (13.17) then the first coordinate $\phi=\left\langle b_{1}, \Phi\right\rangle$ is a solution of (13.14). Since the linear map $\phi \rightarrow \Phi=\sum_{i} b_{i}(\phi) b_{i}^{*}$ is clearly injective we see by a dimension count that these linear maps are inverse isomorphisms between the solution spaces of these two systems of differential equation.

REMARK 13.16. Since the local solution space of an integrable connection at a regular point $p$ can be identified with the fiber of the underlying vector bundle at $p$, the above gives an isomorphism (depending on $p$ ) between the local solution space $\mathcal{L}_{p}(\mu, m)$ of (13.14) at $p$ and the complex vector space $U$.

We claim that the system (13.17) has simple singularities at $\epsilon=0$. The basis vectors $b_{i}=\epsilon^{\operatorname{deg}\left(q_{i}\right)} \partial\left(q_{i}\right)$ have homogeneous degree zero and thus belong to the ring $\mathcal{D}_{0}$ of holomorphic differential operators on $\mathbb{D}^{n}$ generated by vector fields tangent to $\epsilon=0$ (i.e. by $\partial / \partial z_{i}$ with $i>1$ and by $\epsilon \partial / \partial \epsilon$ ). Therefore our claim is easily implied by (also compare to [HeO, Prop. 3.2]).

Lemma 13.17. Given $B \in \mathcal{D}_{0}$ there exists a unique section $u(B)_{\mu, m}=$ $\sum_{j} u(B)_{\mu, m}^{j} b_{j} \in \mathcal{O}\left(\mathbb{D}^{n}\right) \otimes U^{*}$ such that

$$
B \in u(B)_{\mu, m}+\mathcal{I}_{\mu, m} .
$$

The map $\mathcal{D}_{0} \ni B \rightarrow u(B)_{\mu, m}$ is an $\mathcal{O}\left(\mathbb{D}^{n}\right)$-module morphism which depends polynomially on $\mu$ and $m$. For all $B \in \mathcal{D}_{0},\left.u(B)_{\mu, m}\right|_{\{0\} \times \mathbb{D}^{n-1}}$ is independent of $\mu$.

Proof. We use induction on the order $d$ of $B$. Using the well-known theorem that $\mathbb{C}\left[\mathfrak{a}_{\mathbb{C}}{ }^{*}\right]$ is the free $\mathbb{C}\left[\mathfrak{a}_{\mathbb{C}}{ }^{*}\right]^{W}$-module generated by the $W$-harmonic polynomials, we have a unique decomposition

$$
B=\sum_{i, j} f_{i, j}\left(\epsilon, z^{\prime}\right) b_{i} \epsilon^{d_{i, j}} \partial\left(p_{i, j}\right)
$$


with $p_{i, j} \in \mathbb{C}\left[\mathfrak{a}_{\mathbb{C}}\right]^{W}$ a homogeneous polynomial of degree $d_{i, j}$ such that $d_{i, j}+\operatorname{deg}\left(b_{i}\right) \leq d$, and where $f_{i, j}\left(\epsilon, z^{\prime}\right)$ is holomorphic for all $i, j$. Now $\epsilon^{d_{i, j}} \partial\left(p_{i, j}\right)=\epsilon^{d_{i, j}}\left(D_{p_{i, j}}-\gamma\left(D_{p_{i, j}}\right)(\mu)\right)$ modulo lower-order operators in $\mathcal{D}_{0}$, where we have used the fact that for $p \in \mathbb{C}\left[\mathfrak{a}_{\mathbb{C}}\right]^{W}$ homogeneous, the lowest homogeneous part $h^{\eta}\left(D_{p}\right)$ at $\eta$ of $D_{p} \in \mathcal{R}_{m}$ contains the highest-order term $\partial(p)$ of $D_{p}$ (see [O1]). By the induction hypothesis we conclude the existence $u(B)_{\mu, m}$. Using the independence of the $W$-harmonic polynomials over the ring $\mathbb{C}\left[\mathfrak{a}_{\mathbb{C}}{ }^{*}\right]^{W}$ and the induction hypothesis the uniqueness of $u(B)_{\mu, m}$ follows too. By induction and using the fact that the operators $D_{p}$ depend polynomially on $\mu$ and $m$ we conclude that $u(B)_{\mu, m}$ is holomorphic on $\mathbb{D}^{n}$ and polynomial in $\mu$ and $m$. Since in the induction step $\mu$ only occurs via the terms of the form $\epsilon^{d_{i, j}} \gamma\left(D_{p_{i, j}}\right)(\mu)$ we see that $\mu$ does not influence the evaluation at $\epsilon=0$ of $u(B)_{\mu, m}$.

Let $R_{m}$ be the residue matrix of $A_{\mu, m}^{1}$ at $z_{1}=0$. By the previous lemma $R_{m}$ is independent of $\mu$ and is polynomial in $m$. As is well known (cf. [Bo et al, Ch. IV , §4] or [D1]) $R_{m}$ is independent of the coordinate map $i$. Moreover, let us consider on $V=i\left(\{0\} \times \mathbb{D}^{n-1}\right)$ the integrable connection defined by the restrictions $B_{\mu, m}^{i}:=\left.A_{\mu, m}^{i}\right|_{V}$ for $i>1$. Then the residue $R_{m}$ is known to be flat for this integrable connection on $V$. In particular, its characteristic equation is independent of $z^{\prime}$.

Theorem 13.18. The exponents of (13.14) at $\eta$ are the eigenvalues of the residue matrix $R_{m}$ of $A_{\mu, m}^{1}$ at $z_{1}=0$. The characteristic polynomial of $R_{m}$ is independent of $\mu$ and of $z^{\prime}$ and has polynomial coefficients in the $m_{\alpha}$.

Proof. By changing the basis of the trivial vector bundle (with fiber $U$ ) on $i\left(\mathbb{D}^{n}\right)$ by a suitable invertible matrix depending on $z^{\prime}$ only we may assume that $B_{m}^{i}=0$ on $V$ for all $i>1$. We denote the finite-dimensional complex vector space of sections spanned by this basis of flat sections $\mathcal{U}$. By the flatness of $R_{m}$ for the restricted connection on $V$ as above, $R_{m}$ is constant in this new basis (i.e. independent of $z^{\prime}$ ). Let $s$ be an eigenvalue of $R_{m}$, and let $v$ be a generalized $R_{m}$-eigenvector with eigenvalue $s$. Put $u(\epsilon)=\exp \left(\log (\epsilon) R_{m}\right) v=\epsilon^{s} \exp \left(\log (\epsilon)\left(R_{m}-s\right.\right.$ Id $\left.)\right) v$, and observe that

$$
q_{0}^{(s, v)}\left(\epsilon, z^{\prime}\right):=\exp \left(\log (\epsilon)\left(R_{m}-s \operatorname{Id}_{\mathcal{U}}\right)\right) v
$$

is a $\mathcal{U}$-valued polynomial in $\log (\epsilon)$. We denote the series expansion of $\epsilon A_{\mu, m}^{1}$ in $\epsilon$ with respect to a fixed basis of $\mathcal{U}$ by

$$
\epsilon A_{\mu, m}^{1}\left(\epsilon, z^{\prime}\right)=R_{m}+\sum_{k>1} \epsilon^{k} A_{\mu, m, k}^{1}\left(z^{\prime}\right)
$$

with $A_{\mu, m, k}^{1}\left(z^{\prime}\right)$ holomorphic for $z^{\prime} \in \mathbb{D}^{n-1}$. Now we use the following 
relative version of [W, Ch. IV, $\S 24$, Hilfssatz XI]: If $q_{i}\left(\epsilon, z^{\prime}\right)(i<k)$ are $\mathcal{U}$ valued polynomials in $\log (\epsilon)$ of degree $\leq N$ with coefficients in the ring of holomorphic functions on $\mathbb{D}^{n-1}$, then the equation

$$
\epsilon \frac{\partial q_{k}}{\partial \epsilon}+\left((s+k) \operatorname{Id}_{\mathcal{U}}-R_{m}\right) q_{k}=\sum_{i=0}^{k-1} A_{\mu, m, k-i}^{1}\left(z^{\prime}\right) q_{i}
$$

has at least one solution $q_{k}$ which is polynomial in $\log (\epsilon)$ and has coefficients in the ring of holomorphic functions in $z^{\prime} \in \mathbb{D}^{n-1}$. The solution $q_{k}$ is unique and has degree $\leq N$ if $(s+k)$ is not an eigenvalue of $R_{m}$. In general there exist several solutions $q_{k}$ which are polynomial in $\log (\epsilon)$ and these solutions are all of degree $\leq N+r$ in $\log (\epsilon)$, where $r$ is the maximal length of a Jordan block of $R_{m}$ with eigenvalue $(s+k)$.

Given a set $\left\{q_{k}^{(s, v)}\right\}$ of solutions of the recurrence relations (13.23) (with $q_{k}^{(s, v)}$ polynomial in $\log (\epsilon)$ for all $k$, and $q_{0}^{(s, v)}$ given by $\left.(13.21)\right)$ there exists a convergent (but multivalued) series solution $\Phi^{(s, v)}$ of (13.17) on $i\left(\mathbb{D}^{\times} \times \mathbb{D}^{n-1}\right)$ of the form

$$
\Phi^{(s, v)}\left(\epsilon, z^{\prime}\right)=\epsilon^{s} \sum_{k \geq 0} \epsilon^{k} q_{k}^{(s, v)}\left(\epsilon, z^{\prime}\right)
$$

(see, e.g. [W, Ch. IV, $\S 24, \mathrm{XII}])$. Notice that the degree of $q_{k}^{(s, v)}\left(\epsilon, z^{\prime}\right)(k \geq 0)$ as a polynomial in $\log (\epsilon)$ with coefficients in the ring of holomorphic functions in $z^{\prime} \in \mathbb{D}^{n-1}$ is uniformly bounded.

Such a series expansion is not necessarily unique, but by choosing such series solutions $\Phi^{(s, v)}$ for a set of pairs $(s, v)$ where $s$ runs through the set of eigenvalues of $R_{m}$ and, for each $s, v$ runs through a basis of the generalized $s$-eigenspace of $R_{m}$ then the collection of multivalued solutions $\Phi^{(s, v)}$ on $i\left(\mathbb{D}^{\times} \times \mathbb{D}^{n-1}\right)$ constitutes a basis for the space of multivalued solutions of (13.17). On the other hand we have seen above that the flat sections on $i\left(\mathbb{D}^{\times} \times \mathbb{D}^{n-1}\right)$ all are of the form $\Phi=\sum_{i} b_{i}(\phi) b_{i}^{*}$ where $\phi=\left\langle b_{1}, \Phi\right\rangle$ is a solution of (13.14). Hence the set of exponents of (13.14) must coincide with the set of eigenvalues of $R_{m}$, counted with multiplicity.

As a result of the above theorem the following definition makes sense.

Definition 13.19. Let $R_{\mathbf{m}}=R_{\mathbf{m}}^{\eta}$ denote the $|W| \times|W|$-matrix with coefficients in the ring $\mathbb{C}\left[\mathbf{m}_{\alpha}\right] \otimes \mathcal{O}\left(\mathbb{D}^{n-1}\right)$ such that $R_{m}=R_{m}^{\eta}$ is the specialization of $R_{\mathbf{m}}^{\eta}$ at $\mathbf{m}=m$ (this matrix depends on the coordinate map $i$ ). We call the characteristic polynomial $I_{\mathbf{m}}^{\eta} \in \mathbb{C}\left[\mathbf{m}_{\alpha}\right][X]$ of $R_{\mathbf{m}}^{\eta}$ the "indicial polynomial" of (13.14) at $\eta$. 
Corollary 13.20. The (multi-)set $E^{\eta}$ of exponents of (13.14) at $\eta$ is equal to the (multi-)set of roots of the indicial polynomial $I_{m}^{\eta}$ of (13.14) at $\eta$.

13.5 Hecke algebras and exponents. We now bring into play wellknown results on the monodromy of the system of hypergeometric differential equations. We have quite good control for generic parameters as a consequence of the main result, the fact that this representation of the affine braid group factors through an affine Hecke algebra. We apply these results to prove that the indicial polynomial $I^{\eta}$ at $\eta$ factors completely over the ring of rational polynomials in the indeterminates $\mathbf{m}_{\alpha}$ with roots that are affine linear functions in the $\mathbf{m}_{\alpha}$ with half integral coefficients.

By affine Weyl group symmetry we may assume without loss of generality that $\eta \in \bar{\Omega} \cap C$, the fundamental alcove. From now on we will make this assumption.

By Corollary 13.7 the generalized eigenvalue spectrum of $\mathcal{L}_{p}(\mu, m)$ under the action of $T_{p}^{\eta}$ contains information on the set $E^{\eta}$ of exponents of (13.14). Since by (13.4) $T_{p}^{\eta}$ is certainly central in $\Pi_{1}\left(N^{\text {reg }}, p\right)$, the decomposition of $\mathcal{L}_{p}(\mu, m)$ in indecomposable blocks for the monodromy action of $\Pi_{1}\left(N^{\text {reg }}, p\right)$ on $\mathcal{L}_{p}(\mu, m)$ refines the decomposition in generalized $T_{p}^{\eta}$ eigenspaces (by virtue of Schur's lemma).

Therefore we now recall some fundamental facts on the monodromy representation of the fundamental group $\Pi_{1}\left(W^{a} \backslash \mathfrak{a}_{\mathbb{C}}{ }^{\text {reg }}, p\right.$ ) (at a regular base point $p \in \mathfrak{a}_{\mathbb{C}}$ reg in the fundamental alcove $\bar{\Omega} \cap C$ ) on the local solution space $\mathcal{L}_{p}=\mathcal{L}_{p}(\mu, m)$ of (13.14). By a well-known result of Looijenga and Van der Lek ([Le], also see [HeO], [HeS], [O5]) the group $\Pi_{1}\left(W^{a} \backslash \mathfrak{a}_{\mathbb{C}}{ }^{\text {reg }}, p\right)$ is isomorphic to the affine braid group $B^{a}$ of $W^{a}=W \ltimes Q\left(\Sigma^{\vee}\right)$, the affine Weyl group of the affine root system $\Sigma^{a}=\Sigma^{l} \times \mathbb{Z}$. In order to formulate the result we need to define an affine root multiplicity function $m^{a}$ on the affine roots in $\Sigma^{a}$ as follows. For the affine simple roots $a_{0}=1-\theta, a_{1}=\alpha_{1}, \ldots, a_{n}=\alpha_{n}$ we define

$$
\begin{aligned}
& m_{a_{0}}^{a}=m_{\theta}, \\
& m_{a_{i}}^{a}=m_{\alpha_{i}}+m_{\alpha_{i} / 2},
\end{aligned}
$$

and then we extend this to $\Sigma^{a}$ by $W^{a}$-invariance.

Theorem 13.21 (cf. [HeO], [HeS], [O5]). The monodromy action on the $W^{a}$-equivariant local system $\mathcal{L}_{p}(\mu, m)$ on $\mathfrak{a}_{\mathbb{C}}{ }^{\text {reg }}$ factors through an affine Hecke algebra $H\left(W^{a}, q^{a}\right)$ in the following sense.

Let $q^{a}$ be the label function on the affine root system $\Sigma^{a}=\Sigma^{l} \times \mathbb{Z}$ defined by $q_{b}^{a}=\exp \left(-\pi m_{b}^{a}\right)$ for all $b \in \Sigma^{a}$. For the simple affine roots $a_{i}$ 
we write $q_{a_{i}}^{a}:=q_{i}^{a}$. The monodromy matrices $M_{\mu, m}\left(b_{i}\right)(i=0, \ldots, n)$ of the generators $b_{i}$ of $B^{a}$ satisfy $\left(M_{\mu, m}\left(b_{i}\right)-1\right)\left(M_{\mu, m}\left(b_{i}\right)+q_{i}^{a}\right)=0$. The monodromy representation $M_{\mu, m}$ of $\Pi_{1}\left(W^{a} \backslash \mathfrak{a}_{\mathbb{C}}{ }^{\text {reg }}, p\right)$ depends analytically on the parameters $m$ and $\mu$.

Recall that $W_{\eta}^{a}$ is the isotropy subgroup of $\eta$ in $W^{a}$, which is a finite reflection group, and let $\Sigma_{\eta}^{a}$ be the corresponding root system. There is a natural monomorphism $W_{\eta}^{a} \rightarrow W$ with image $\tilde{W}_{\eta}^{a} \subset W$. We put $N_{\eta}=$ $\left[W: \tilde{W}_{\eta}^{a}\right]$ for the index of this subgroup.

Let us denote by $B_{\eta}^{a} \subset B^{a}$ the braid group of $W_{\eta}^{a}$, which we can identify, by Brieskorn's theorem on the fundamental group of the regular orbit space of a finite reflection group, with the fundamental group of the "local regular orbit space" at $\eta$, namely $\Pi_{1}\left(W_{\eta}^{a} \backslash N^{\text {reg }}, p\right)$.

Let $m_{\eta}^{a}$ be the restriction of $m^{a}$ to $\Sigma_{\eta}^{a}$, and let $q_{\eta}^{a}$ be corresponding the corresponding root multiplicity function on $\Sigma_{\eta}^{a}$. Let $\mathcal{Q}$ denote the finitedimensional complex vector space of complex multiplicity functions $m$ on (the possibly non-reduced) root system $\Sigma$. In a dense, open set $\mathcal{Q}_{\eta}^{\text {reg }} \subset \mathcal{Q}$ of values of the parameter $m$, the finite-dimensional Hecke algebra $H\left(W_{\eta}^{a}, q_{\eta}^{a}\right)$ (with $q_{\eta}^{a}=q\left(m_{\eta}^{a}\right)$ ) is a semisimple algebra. If we assume that $m \in \mathcal{Q}_{\eta}^{\text {reg }}$ then, by Tits' deformation lemma, we can index its set of irreducible modules by $\widehat{W_{\eta}^{a}}$, the set of irreducible representations of $W_{\eta}^{a}$. Given $\tau \in \widehat{W_{\eta}^{a}}$ and $m \in \mathcal{Q}_{\eta}^{\text {reg }}$ we will write $\pi_{\tau}^{\eta}\left(q_{\eta}^{a}\right)$ for the corresponding irreducible $H\left(W_{\eta}^{a}, q_{\eta}^{a}\right)$ module. Upon restriction of the monodromy action of $B^{a}$ on $\mathcal{L}_{p}(\mu, m)$ to $B_{\eta}^{a}$ we have

Corollary 13.22. Let $q=q(m)$ and $q_{\eta}^{a}=q\left(m_{\eta}^{a}\right)$ for $m \in \mathcal{Q}_{\eta}^{\text {reg }}$. The monodromy action of $\Pi_{1}\left(W_{\eta}^{a} \backslash N^{\mathrm{reg}}, p\right)$ on $\mathcal{L}_{p}(\mu, m)$ factors through the semisimple finite-type Hecke algebra $H\left(W_{\eta}^{a}, q_{\eta}^{a}\right)$, and the local solution space $\mathcal{L}_{p}(\mu, m)$ decomposes under this action in isotypical components

$$
\mathcal{L}_{p}(\mu, m)=\bigoplus_{\tau \in \widehat{W_{\eta}^{a}}} \mathcal{L}_{p}(\mu, m)(\tau)
$$

such that for each $\tau \in \widehat{W_{\eta}^{a}}, \mathcal{L}_{p}(\mu, m)(\tau) \simeq K(\tau, m) \otimes \pi_{\tau}^{\eta}\left(q_{\eta}^{a}\right)$ with $\operatorname{dim}(K(\tau, m))=N_{\eta} \operatorname{deg}_{\tau}$ (independent of $m \in \mathcal{Q}_{\eta}^{\text {reg }}$ ).

Proof. Using the rigidity of semisimple finite dimensional algebras (Tits' deformation lemma, [C, Prop. 10.11.4]) the multiplicity of $\pi_{\tau}^{\eta}\left(q_{\eta}^{a}\right)$ is constant in $(\mu, m) \in \mathfrak{a}_{\mathbb{C}}{ }^{*} \times \mathcal{Q}_{\eta}^{\text {reg }}$. We may therefore compute the multiplicity by evaluating at $(\mu, m)=(0,0)$. Hence it is equal to the multiplicity of $\tau$ in the restriction of the regular representation of $W$ to $\tilde{W}_{\eta}^{a}$, which is $N_{\eta} \operatorname{deg}_{\tau}$. $\square$ 
The following topological observation due to Deligne [D2] is crucial for our purpose:

LEMmA 13.23. Let $\beta_{p}^{\eta} \in B_{\eta}^{a}$ denote the local braid in $\Pi_{1}\left(W_{\eta}^{a} \backslash N^{\mathrm{reg}}, p\right)$ which corresponds to a reduced expression of the longest element of $W_{\eta}^{a}$. Then $\left(\beta_{p}^{\eta}\right)^{2}=\left[\gamma_{p}^{\eta}\right]$ (see Definition 13.4). In particular this element is central in $B_{\eta}^{a}$.

Given $\tau \in \widehat{W_{\eta}^{a}}$ we denote by $p_{\eta, \tau}^{i}$ (with $i=1, \ldots, N_{\eta} \operatorname{deg}_{\tau}$ ) the embedding degrees of $\tau$ in the graded vector space of $W$-harmonic polynomials. We choose these $W$-harmonic embedding degrees so that $i \rightarrow p_{\eta, \tau}^{i}$ is a nondecreasing sequence. In particular, $p_{\eta, \tau}^{1}$ is the "harmonic birthday" of $\tau$ in the $W$-harmonic polynomials.

Theorem 13.24. Let $\tau \in \widehat{W_{\eta}^{a}}$ and let $m \in \mathcal{Q}_{\eta}^{\text {reg }}$. The multiset $E^{\eta}(\tau, m)$ of exponents of $\mathcal{L}_{p}(\mu, m)(\tau)$ consists of the complex numbers

$$
s_{\eta, \tau}^{i}(m)=p_{\eta, \tau}^{i}-\frac{1}{2} c_{\eta, \tau}(m),
$$

where $i$ runs from 1 to $N_{\eta} \operatorname{deg}_{\tau}$, each $s_{\eta, \tau}^{i}(m)$ occurring with multiplicity $\operatorname{deg}_{\tau}$. Here $c_{\eta, \tau}(m)$ is the affine linear function of the multiplicity parameters $m_{\alpha}$ with nonnegative integral coefficients defined by (cf. (13.25) for the definition of $\left.m_{\eta, b}^{a}\right)$ :

$$
c_{\eta, \tau}(m)=\sum_{b \in \Sigma_{\eta,+}^{a}}\left(1-\frac{\chi_{\tau}\left(s_{b}\right)}{\operatorname{deg}_{\tau}}\right) m_{\eta, b}^{a} .
$$

Proof. Since $T_{p}^{\eta}$ is the monodromy action of the (locally) central braid $\left(b_{p}^{\eta}\right)^{2}$ (by Lemma 13.23) we see that $T_{p}^{\eta}$ acts trivially in the multiplicity space $K(\tau, m)$ and acts by scalar multiplication in the irreducible representation $\pi_{\tau}^{\eta}\left(q_{\eta}^{a}\right)$ of the Hecke algebra $H\left(W_{\eta}^{a}, q_{\eta}^{a}\right)$ by some scalar $C$. This $C$ is an element of the ring of Laurent polynomial in the Hecke algebra labels $\left(q_{\eta, b}^{a}\right)^{1 / 2}\left(\right.$ with $\left.b \in \Sigma_{\eta}^{a}\right)$ since this is the splitting ring of the Hecke algebra. By taking the determinant of $T_{p}^{\eta}$ in $\pi_{\tau}^{\eta}\left(q_{\eta}^{a}\right)$ we find easily that $C^{\operatorname{deg}_{\tau}}=\exp \left(-i \pi \operatorname{deg}_{\tau} c_{\eta, \tau}(m)\right)$. This implies that $C$ is a root of 1 times a monomial in the $\left(q_{\eta, b}^{a}\right)^{ \pm 1 / 2}$. For $\left(q_{\eta, b}^{a}\right)^{1 / 2}=1$ we have $C=1$, hence

$$
C=\exp (-i \pi c(m)) \text {. }
$$

Let $\mathcal{N}$ denote the collection of functions $\nu$ on the set $\tau \in \widehat{W_{\eta}^{a}}$ which associate to each $\tau$ a finite multiset $\nu(\tau)=\left\{\nu_{\tau, j} \mid j=1, \ldots, N_{\eta} \operatorname{deg}_{\tau}^{2}\right\}$ of $N_{\eta} \operatorname{deg}_{\tau}^{2}$ integers $\nu_{\tau, j} \in \mathbb{Z}$. By Corollary 13.7 and Corollary 13.20 it follows that for each $m \in \mathcal{Q}_{\eta}^{\text {reg }}$ the set of roots of the indicial polynomial $I_{m}^{\eta}$ is a multiset of the form $\rho_{\tau, \nu, j}(m)=\nu_{\tau, j}-1 / 2 c_{\eta, \tau}(m)$ for some $\nu \in \mathcal{N}$. For each $\nu \in \mathcal{N}$ the set $\mathcal{Q}(\nu) \subset \mathcal{Q}$ of multiplicity parameters $m \in \mathcal{Q}$ for which the multiset of 
roots of $I_{m}^{\eta}$ is equal to the multiset $\left\{\rho_{\tau, \nu, j}(m)\right\}$ is Zariski-closed (since $I_{m}^{\eta}$ is a polynomial in $m$, by Corollary 13.20). Moreover the union of these sets contains $\mathcal{Q}_{\eta}^{\text {reg }}$. Since $\mathcal{N}$ is countable, Baire's category theorem implies that there must exist at least one $\nu_{0} \in \mathcal{N}$ such that the interior (in the analytic topology) of $\mathcal{Q}\left(\nu_{0}\right)$ is nonempty, and hence such that $\mathcal{Q}=\mathcal{Q}\left(\nu_{0}\right)$.

In the situation $m \in \mathcal{Q}_{\eta}^{\text {reg }}$ we have the splitting in the isotypical components (13.26). It follows that the set $E^{\eta}(\tau, m)$ consists of the subset $\rho_{\tau, \nu_{0}, j}(m)\left(j=1, \ldots, N_{\eta} \operatorname{deg}_{\tau}^{2}\right)$ of roots of the indicial equation. Finally we need to determine $\nu_{0}(\tau)$. This is resolved by taking $m=0 \in \mathcal{Q}_{\eta}^{\text {reg }}$ and comparing with Example 13.8, after making the additional remark that the monodromy representation $\pi_{\tau}^{\eta}\left(q_{\eta}^{a}\right)$ is by definition equal to $\tau$ if $q_{\eta}^{a}=1$.

Corollary 13.25. For $m \in \mathcal{Q}_{\eta}^{\text {reg }}$ the action of $T_{p}^{\eta}$ is semisimple. In particular, there are no logarithmic terms in the decomposition (13.2) if $m \in \mathcal{Q}_{\eta}^{\text {reg }}$ and if $\phi_{p} \in \mathcal{L}_{p}(\mu, m)$.

So we conclude this subsection with the following remarkable result:

COROLlary 13.26. The indicial polynomial factorizes as

$$
I_{m}(X)=\prod_{\tau \in \widehat{W_{\eta}^{a}}} \prod_{i=1}^{N_{\eta} \operatorname{deg}_{\tau}}\left(X-s_{\eta, \tau}^{i}(m)\right)^{\operatorname{deg}_{\tau}} .
$$

For $m \in \mathcal{Q}^{\text {reg }}$ this factorization is compatible with the decomposition of $\mathcal{L}_{p}(\mu, m)$ in blocks of the form $\mathcal{L}_{p}(\mu, m)(\tau)$ as in Corollary 13.22.

13.6 Computation of the leading exponents. Let $\phi_{\mu, m} \in \mathcal{L}_{p}(\mu, m)$ with $p \in \bar{\Omega} \cap C$ denote the hypergeometric function, the solution of (13.14) whose germ at points of the fundamental alcove $\bar{\Omega} \cap C$ we define by analytic continuation along a path in $\bar{\Omega} \cap C$ of the unique normalized $W$-invariant solution of (13.14) which extends holomorphically to a neighborhood of $0 \in \mathfrak{a}_{\mathbb{C}}$.

COROllary 13.27. By definition, $\phi_{\mu, m}$ extends holomorphically over all finite walls, the walls of $C$, to a $W$-invariant function on $\Omega$, the interior of $W C$. In particular, if $\eta \in \bar{\Omega} \cap C$ and $\theta(\eta) \neq 1$ then $E^{\eta}\left(\phi_{\mu, m}\right)=\{\kappa\}$ with $\kappa \in \mathbb{Z}_{\geq 0}$ (generically $\kappa=0$, of course).

In this section, we will be interested in the case where $\eta=\omega_{j} / k_{k}$ as in Theorem 2.6. In this case we know that $\Sigma_{\eta}^{a}$ is an irreducible root system. From the definition of $\phi_{\mu, m}$ we see that

Corollary 13.28. Let $m \in \mathcal{Q}_{\eta}^{\text {reg }}$. Let $W_{\eta} \subset W_{\eta}^{a}$ be the maximal parabolic subgroup of $W_{\eta}^{a}$ generated by the simple reflections $s_{i}$ of $W$ which 
fix $\eta$. Then $\phi_{\mu, m} \in \mathcal{L}_{p}(\mu, m)$ belongs to the subspace $\mathcal{L}_{p}^{\eta}(\mu, m)$ defined by

$$
\mathcal{L}_{p}^{\eta}(\mu, m):=\bigoplus_{\tau \in J_{\eta}} \mathcal{L}_{p}(\mu, m)(\tau)
$$

where $J_{\eta} \subset \widehat{W_{\eta}^{a}}$ is the subset consisting of irreducible representations which occur in the induction of the trivial representation of $W_{\eta}$ to $W_{\eta}^{a}$.

The above fact restricts the $T_{p}^{\eta}$-spectrum of $\phi_{\mu, m}$, and thus the set of exponents $E^{\eta}\left(\phi_{\mu, m}\right)$, drastically for $m \in \mathcal{Q}^{\text {reg }}$. We assume from now on that $m$ is real valued, which we denote by $m \in \mathcal{Q}(\mathbb{R})$. By Theorem 13.24 the multiset $E^{\eta}\left(\phi_{\mu, m}\right)$ consists of real numbers now.

Definition 13.29. Let $m \in \mathcal{Q}(\mathbb{R})$. We call the smallest element in the the multiset $E^{\eta}\left(\phi_{\mu, m}\right)$ the leading exponent of $\phi_{\mu, m}$ at $\eta$. The irreducible characters $\tau \in J_{\eta} \subset W_{\eta}^{a}$ affording the leading exponent are called leading characters.

Theorem 13.30. If $\Sigma_{\eta}^{a}$ is reduced and simply laced we denote the root multiplicity by $m=m_{1} \geq 1$. In general $m_{1}$ denotes the root multiplicity of the longest roots. The multiplicity of half a long root is denoted by $m_{1 / 2} \geq 0$ (i.e. we consider $C_{n}$ as the special case of $B C_{n}$ where $m_{1 / 2}=0$; since the geometry of $\Omega$ depends on $\Sigma^{l}$ only this is allowed). Let $\eta \in \partial \Omega \cap C$ be an extremal boundary point of $\Omega$ and assume that $m \in \mathcal{Q}(\mathbb{R})$ is in the cone $\mathcal{C} \in \mathcal{Q}(\mathbb{R})$ defined by the inequalities

$$
1 \leq m_{1} \leq m_{2}
$$

(these inequalities are obviously satisfied by the multiplicity function $m^{X}$ of a Riemannian symmetric space $X$ with restricted root system $\Sigma_{X}$ such that $\left.\Sigma_{X}^{l}=\Sigma^{l}\right)$. The leading exponent $s_{\eta}(m)$ of $\phi_{\mu, m}$ at $\eta$ satisfies

$$
s_{\eta}(m) \geq s_{\eta, \tau}^{1}(m)
$$

where

$$
\tau=\sigma_{\eta}=\operatorname{det}_{\eta}^{a} \otimes j_{W_{\eta}}^{W_{\eta}^{a}}\left(\operatorname{det}_{\eta}\right) .
$$

Here $\operatorname{det}_{\eta}^{a}$ is the determinant representation of $W_{\eta}^{a}$, and $\operatorname{det}_{\eta}$ its restriction to $W_{\eta}$. Moreover, for generic $m \in \mathcal{C}$ the inequality (13.33) is an equality and $\sigma_{\eta}$ is a leading character.

Proof. This is based on a case-by-case analysis. We first assume that $m \in \mathcal{Q}_{\eta}^{\text {reg }}(\mathbb{R})$ is regular. We compute in all cases the set $J_{\eta}$ of irreducible components $\tau$ of the induction of the trivial representation of $W_{\eta}$ to $W_{\eta}^{a}$ (which is relatively easy, as $W_{\eta}$ is a rather large subgroup of $W_{\eta}^{a}$ ). In the classical cases we use the Littlewood-Richardson rule, and in the exceptional cases we refer to the character tables in the computer algebra packet 
CHEVIE. We use below the notation for the irreducible characters as used in $[\mathrm{C}])$.

We are lucky: if we consider for each $\pi \in J_{\eta}$ the smallest associated exponent $s_{\eta, \tau}^{1}(m)$ (using Theorem 13.24) we can simply check that these are indeed all greater than or equal to $s_{\eta, \tau}^{1}(m)$, where $\tau=\sigma_{\eta}$ and if $m \in \mathcal{C}$. Recall that $\sigma_{\eta} \in J_{\eta}$ was the term which gave the unique leading exponent in the complex case (see Example 13.10, Proposition 7.8), which corresponds only to one interior point $m^{X_{\mathbb{C}}} \in \mathcal{C}$ of $\mathcal{C}$. In any case, this surprising fact is enough to prove that for generic $m \in \mathcal{C}$ the value $s_{\eta, \tau}^{1}(m)$ really is the leading exponent of $\phi_{\mu, m}$ at $\eta$ by the fact that $\phi_{\mu, m}$ is holomorphic in the parameter $m$ (see Theorem 13.15).

Below will now show these claims in a case-by-case analysis:

Type $A_{l-1}(l \geq 3)$ : For $\Sigma=A_{l-1}$ all the nodes of the Dynkin diagram are minuscule and thus extremal according to Theorem 2.6. Let $\omega_{j}$ be the $j$-th node of the Dynkin diagram. By symmetry we may assume without loss of generality that $1 \leq j \leq l / 2$. Recall that the irreducible characters $\chi_{\lambda}$ of $S_{l}$ are parameterized by the partitions $\lambda$ of $l$ in such a way that $\chi_{l}=1$ and $\chi_{1^{l}}=\epsilon$ (the determinant representation). We denote the i-th exponent corresponding to $\chi_{\lambda}$ by $\sigma_{\lambda}^{i}(m)$.

By the Littlewood-Richardson rule [M, $\S$ I.9] we have

$$
\operatorname{Ind}_{S_{j} \times S_{l-j}}^{S_{l}}\left(\chi_{j} \times \chi_{(l-j)}\right)=\bigoplus_{0 \leq i \leq j} \chi_{(l-i, i)}
$$

and we have that (see $[\mathrm{C}, \S \S 11.2,11.4])$ :

$$
\sigma_{\omega_{j}}:=\epsilon \otimes j_{S_{l-j} \times S_{j}}^{S_{l}}\left(\epsilon_{l-j} \times \epsilon_{j}\right)=\chi_{(l-j, j)} .
$$

Using Theorem 13.24 and standard facts on representations of $S^{l}$ we find that

$$
s_{(l-i, i)}^{1}(m)=i(1-(l+1-i) m / 2) .
$$

Under the condition (13.32) (namely $m \geq 1$ ) we see that among the exponents $s_{(l-i, i)}(m)$ at $\omega_{j}$ (thus with $i \leq j$ ) indeed

$$
s_{\omega_{j}}(m):=s_{(l-j, j)}^{1}(m)=j(1-(l+1-j) m / 2)
$$

is the unique minimal one, unless $l$ is even, $m=1$ and $j=l / 2$. In this last case the two components $(l / 2, l / 2)$ and $(l / 2+1, l / 2-1)$ of $(13.35)$ both have the same exponent $l(l-2) / 8$.

Type $B_{l}(l \geq 3)\left(\eta=\omega_{1}\right)$ : Recall that the irreducible characters $\chi_{(\lambda, \mu)}$ of $B_{l}$ are parameterized by ordered pairs $(\lambda, \mu)$ of partitions of total weight $l$. Here $\chi_{(l, 0)}=1$ and $\chi_{\left(0,1^{l}\right)}=\epsilon$. We have (using the LR rule again for wreath products, see [M, I.App.B]): 


$$
\begin{aligned}
\operatorname{Ind}_{B_{l-1}}^{B_{l}} & \left(\chi_{(l-1,0)}\right) \\
& =\operatorname{Ind}_{B_{l-1} \times B_{1}}^{B_{l}}\left(\chi_{(l-1,0)} \times \chi_{(1,0)}\right)+\operatorname{Ind}_{B_{l-1} \times B_{1}}^{B_{l}}\left(\chi_{(l-1,0)} \times \chi_{(0,1)}\right) \\
& =\chi_{(l, 0)}+\chi_{(l-1,1)}+\chi_{((l-1,1),-)}
\end{aligned}
$$

and thus

$$
J_{\omega_{1}}=\left\{\chi_{(l, 0)}, \chi_{(l-1,1)}, \chi_{((l-1,1),-)}\right\} .
$$

From [C, Prop. 11.4.2] we find

$$
\sigma_{\omega_{1}}:=\epsilon_{l} \otimes j_{B_{l-1}}^{B_{l}}\left(\chi_{\epsilon_{l-1}}\right)=\epsilon_{l} \otimes \chi_{\left(1,1^{l-1}\right)}=\chi_{(l-1,1)}
$$

and the birthday of $\chi_{\left(1,1^{l-1}\right)}$ is $\left|\Sigma\left(B_{l-1}\right)_{+}\right|=(l-1)^{2}$.

Using Theorem 13.24 and standard results on representations of $W\left(B_{l}\right)$ (e.g. [C, Ch. 11]) we find

$$
\begin{aligned}
& s_{(l-1,1)}^{1}\left(m_{2}, m_{1}\right)=1-m_{2}-(l-1) m_{1}, \\
& s_{((l-1,1),-)}^{1}\left(m_{2}, m_{1}\right)=2-l m_{1} .
\end{aligned}
$$

Under the condition (13.32) (i.e. if $1 \leq m_{1} \leq m_{2}$ ) then we see that the first one is indeed always smaller than the second one.

Type $B_{l}(l \geq 3) \quad\left(\eta=\omega_{l} / 2\right)$ : Not minuscule, with $W_{\eta}^{a}=W\left(D_{l}\right)$ and $W_{\eta}=S_{l}$, so this reduces to the minuscule case $D_{l}, \eta=\omega_{l}\left(\right.$ with $\left.m=m_{1}\right)$ if $l \geq 4$, or to $A_{3}, \eta=\omega_{1}$ if $l=3$.

Types $B C_{l}(l \geq 1)$ and $C_{l}(l \geq 2)$ : We treat these cases together, since the geometry of $\Omega$ is the same.

We have one boundary orbit to consider, namely $\eta=\omega_{l}$, a minuscule case. We have $W_{\eta}^{a}=W\left(C_{l}\right)$ and $W_{\eta}=W\left(A_{l-1}\right)=S_{l}$, with root multiplicities $m_{a_{0}}^{a}=m_{1}$ for the long roots of $C_{l}$, and $m_{2}$ for the short roots of $C_{l}$.

In the construction of [C, Prop. 11.4.2] it is easy to see that the irreducible character $\chi_{(i, l-i)}$ of $W\left(C_{l}\right)$ is realized on the space of polynomials in $\mathbb{C}\left[x_{1}, \ldots, x_{n}\right]$ by the action of $W\left(C_{l}\right)$ on the monomial $x_{1} \ldots x_{l-i}$ $(i=0, \ldots, l)$. Hence this character contains the trivial character of $S_{l}$ and has dimension binomial $(l, i)$, and has its birthday in degree $l-i$. By dimension count we find that

and so

$$
\operatorname{Ind}_{S_{l}}^{W\left(C_{l}\right)}\left(\chi_{l}\right)=\oplus_{i=0}^{l} \chi_{(i, l-i)}
$$

$$
J_{\omega_{l}}=\left\{\left(\chi_{(i, l-i)}\right\}_{i=0}^{l} a .\right.
$$

We also see easily from the above realization that

$$
s_{(i, l-i)}^{1}(m)=(l-i)\left(1-m_{1}-i m_{2}\right)
$$

The characters $\epsilon \otimes \chi_{(i, l-i)}=\chi_{\left(1^{l-i}, 1^{i}\right)}$ all contain the sign representation of $S_{l}(i=0, \ldots, l)$; thus together they fill up (multiplicity free) the character 
of $W\left(C_{l}\right)$ induced from the sign representation of $S_{l}$. According to [C, Prop. 11.4.2] the birthday of $\chi_{\left(1^{l-i}, 1^{i}\right)}$ is in degree $\left|\Sigma\left(D_{l-1}\right)_{+}\right|+\left|\Sigma\left(C_{i}\right)_{+}\right|=$ $l(l-1)+i((2 l-1)-2 i)$. We see that the minimum is attained for $i=r$ if $l=2 r$ or if $l=2 r+1$. Hence if $l=2 r$ we get

$$
\sigma_{\omega_{2 r}}=\chi_{(r, r)}
$$

whereas in the case $l=2 r+1$ we have

$$
\sigma_{\omega_{2 r+1}}=\chi_{(r, r+1)}
$$

CASE $l=2 r(r \geq 1)$ EVEN: One checks that

$$
s_{(i, 2 r-i)}^{1}(m)-s_{(r, r)}^{1}(m)=(r-i)\left((r-i) m_{2}-m_{1}+1\right)
$$

which is strictly positive on $\mathcal{C}$ for $0 \leq i \leq 2 r$ and $i \neq r$.

CASE $l=2 r+1(r \geq 0)$ ODD: One checks that

$$
s_{(i, 2 r-i+1)}^{1}(m)-s_{(r, r+1)}^{1}(m)=(r-i)\left((r-i+1) m_{2}-m_{1}+1\right) .
$$

For $0 \leq i \leq 2 r+1$ and $i \neq r$ this is nonnegative on $\mathcal{C}$, and it is zero precisely when $m_{1}=1$ and $i=r+1$. Observe that this is also true if $r=0$.

Type $D_{l}(l \geq 4), \eta=\omega_{1}$ : This is a minuscule case. Recall that the irreducible characters $\chi_{(\lambda, \mu)}$ of $D_{l}$ are parameterized by unordered pairs $(\lambda, \mu)$ of partitions of total weight $l$ where $\lambda \neq \mu$, and characters $\chi_{(\lambda, \lambda)}^{\prime}, \chi_{(\lambda, \lambda)}^{\prime \prime}$ if $l$ is even (weight of $\lambda$ is $l / 2$ ). The character $\chi_{(\lambda, \mu)}$ is the restriction of the character of $W\left(B_{l}\right)$ with the same label $(\lambda, \mu)$ to $W\left(D_{l}\right)$. This restriction stays irreducible unless $\lambda=\mu$, in which case the character splits as a sum of two irreducible characters which we distinguish by ' and ". Thus $\chi_{(l, 0)}=1$ and $\chi_{\left(1^{l}, 0\right)}=\epsilon$.

By restriction of (13.39) to $W\left(D_{l}\right)$ we find

$$
J_{\omega_{1}}=\left\{\chi_{(l, 0)}, \chi_{(l-1,1)}, \chi_{((l-1,1),-)}\right\} .
$$

From [C, Prop. 11.4.2] we find

$$
\sigma_{\omega_{1}}:=\epsilon_{l} \otimes j_{D_{l-1}}^{D_{l}}\left(\chi_{\epsilon_{l-1}}\right)=\epsilon_{l} \otimes \chi_{\left(\left(2,1^{l-1}\right),-\right)}=\chi_{((l-1,1),-)}
$$

where the birthday of $\chi_{\left(\left(2,1^{l-1}\right),-\right)}$ is in $\left|\Sigma\left(D_{l-1}\right)_{+}\right|=(l-1)(l-2)$.

Using Theorem 13.24 and standard results on representations of $W\left(D_{l}\right)$ (e.g. [C, Ch.11]) we find (one should compare this to (13.42))

$$
\begin{aligned}
& s_{(l-1,1)}^{1}(m)=1-(l-1) m, \\
& s_{((l-1,1),-)}^{1}(m)=2-l m .
\end{aligned}
$$

Under the condition (13.32) (i.e. if $1 \leq m$ ) then we see indeed that the second one is smaller than the first one, except in the case $m=1$ when they coincide.

Type $D_{l}(l \geq 4), \eta=\omega_{l}$ : This is minuscule too. For the computation of $J_{\omega_{l}}$ we recall the realizations for the characters $\chi_{(l-i, i)}$ as described in 
the text above (13.43). We introduce an intertwining operator $\mathcal{J}$ for the restriction of these representations to $W\left(D_{l}\right)$. If $\Omega \subset\{1, \ldots, l\}$ we denote by $x_{\Omega}$ the product of the $x_{i}$ with $i \in \Omega$. We now define $\mathcal{J}\left(x_{\Omega}\right)=$ $x_{\Omega^{c}}$ and extend by linearity. Then $\mathcal{J}$ is an intertwining isomorphism $\mathcal{J}:\left.\left.\pi_{(\alpha, \beta)}\right|_{W\left(D_{l}\right)} \rightarrow \pi_{(\beta, \alpha)}\right|_{W\left(D_{l}\right)}$, and if $\alpha=\beta$ then $\mathcal{J}$ splits $\left.\pi_{(\alpha, \alpha)}\right|_{W\left(D_{l}\right)}$ in $\pi_{(\alpha, \alpha)}^{\prime}($ the +1 -eigenspace of $\mathcal{J})$ and $\pi_{(\alpha, \alpha)}^{\prime \prime}$ (the -1 -eigenspace of $\left.\mathcal{J}\right)$. Thus $\pi_{(\alpha, \alpha)}^{\prime}$ contains the $S_{l}$-spherical vector with this convention. Hence if $l=2 r$ then

and if $l=2 r+1$ then

$$
J_{\omega_{l}}=\left\{\chi_{(r, r)}^{\prime}\right\} \cup\left\{\chi_{(i, 2 r-i)}\right\}_{i=r+1}^{2 r}
$$

$$
J_{\omega_{l}}=\left\{\chi_{(i, 2 r-i+1)}\right\}_{i=r+1}^{2 r+1} .
$$

As in the text below (13.43) we find that if $l=2 r+1$ then

whereas if $l=2 r$ then

$$
\sigma_{\omega_{2 r+1}}=\chi_{(r+1, r)}
$$

$$
\sigma_{\omega_{2 r}}=\chi_{(r, r)}^{\prime} .
$$

In the odd case $l=2 r+1$ we thus get the specialization of the result (13.43) for $C_{l}$ at $m_{1}=0$, namely,

$$
s_{\chi_{(2 r+1-i, i)}}^{1}(m)=(2 r+1-i)(1-i m),
$$

but this time this has a unique minimal value among $J_{\omega_{l}}$ at $i=r+1$ (which proves our claim in this case, in view of (13.55)). Hence $s_{\omega_{2 r+1}}=$ $r(1-(r+1) m)$.

In the even case we need to look more closely at our model for $\chi_{(r, r)}^{\prime}$ first. The degree of this representation is $\operatorname{binomial}(2 r, r) / 2=$ binomial $(2 r-1, r-1)$. The dimension of the -1 eigenspace of a reflection is equal to $\operatorname{binomial}(2 r-2, r-1) / 2=\operatorname{binomial}(2 r-3, r-2)$. This leads to

$$
s_{\chi_{(r, r)}}^{1}(m)=r(1-r m)
$$

which is still same the same answer as we had in $C_{2 r}$ when substituting $m_{1}=0$ (cf. (13.45)). Therefore this exponent indeed represents the unique minimal exponent among those associated with the characters in $J_{\omega_{1}}$ proving the claim in this case as well.

Type $E_{6}, \eta=\omega_{1}$ : This is minuscule. By the character tables in "CHEVIE" we find that

$$
J_{\omega_{1}}=\left\{\chi_{1,0}, \chi_{6,1}, \chi_{20,2}\right\}
$$

and

$$
\begin{aligned}
& s_{6,1}^{1}(m)=1-6 m, \\
& s_{20,2}^{1}(m)=2-9 m .
\end{aligned}
$$


The second one is the unique minimal exponent, and we check that $s_{20,2}^{1}(2)=$ $-16=\left|\Sigma\left(D_{5}\right)_{+}\right|-\left|\Sigma\left(E_{6}\right)_{+}\right|$. In view of Proposition 7.8 this proves the claims in this case.

Type $E_{7}, \eta=\omega_{7}$ : This is minuscule. By the character tables in "CHEVIE" we find that

$$
J_{\omega_{1}}=\left\{\chi_{1,0}, \chi_{7,1}, \chi_{27,2}, \chi_{21,3}\right\}
$$

and

$$
\begin{aligned}
& s_{7,1}^{1}(m)=1-9 m, \\
& s_{27,2}^{1}(m)=2-14 m, \\
& s_{21,3}^{1}(m)=3-15 m .
\end{aligned}
$$

The last one is the unique minimal exponent, except when $m=1$ when it coincides with the second one. We check that $s_{21,3}^{1}(2)=-27=$ $\left|\Sigma\left(E_{6}\right)_{+}\right|-\left|\Sigma\left(E_{7}\right)_{+}\right|$. In view of Proposition 7.8 this proves the claims.

Type $E_{7}, \eta=\omega_{2} / 2$ : This is not minuscule, and reduces to the case $\left(A_{7}\right.$, $\left.\eta=\omega_{1}\right)$.

Type $E_{8}, \eta=\omega_{1} / 2$ : This is not minuscule, and reduces to the case $\left(D_{8}, \omega_{1}\right)$.

Type $E_{8}, \eta=\omega_{2} / 3$ : This is not minuscule, and reduces to the case $\left(A_{8}, \omega_{1}\right)$.

Type $F_{4}$ : This is not minuscule, and reduces to the case $\left(B_{4}, \omega_{1}\right)$.

Type $G_{2}$ : This is not minuscule, and reduces to the case $\left(A_{2}, \omega_{1}\right)$.

Corollary 13.31 (of the proof of the previous theorem). For $m \in \partial \mathcal{C}$ the boundary of $\mathcal{C}$ there are at most two inequivalent irreducibles $\tau, \pi \in J^{\eta}$ such that $s_{\eta, \tau}^{1}(m)=s_{\eta, \pi}^{1}(m)$. The cases where this occurs are indicated in the last column of the table of Theorem 7.9.

Corollary 13.32. Let $m \in \partial \mathcal{C}$ be such that there are two inequivalent irreducible representations $\tau, \pi \in J^{\eta}$ with coinciding exponents $s_{\eta, \tau}^{1}(m)=$ $s_{\eta, \pi}^{1}(m)$. Then the term of (13.2) corresponding to the leading exponent $s_{\eta, \tau}^{1}(m)$ contains possibly a $\log (\epsilon)$ term of degree at most one. Otherwise the leading term in (13.2) has no logarithmic term.

Proof. This is an easy consequence of Corollary 13.25 and the fact that $\phi_{\mu, m}$ is holomorphic in $m$. Indeed, suppose that an expression of the form (with $s \in \mathbb{R} \backslash\{0\}$ fixed)

$$
f(m, \epsilon)=a(m, \epsilon)+b(m, \epsilon) \epsilon^{s m}
$$


is a local Nilsson class function of $(m, \epsilon) \in \mathbb{D} \times \mathbb{D}^{\times}$with $a(m, \epsilon), b(m, \epsilon)$ both holomorphic for $\epsilon \in \mathbb{D}$ for all fixed $m \in \mathbb{D}^{\times}$. Then analytic continuation around $\epsilon=0$ implies that

$$
f^{\prime}(m, \epsilon)=a(m, \epsilon)+\exp (2 i \pi s m) b(m, \epsilon) \epsilon^{s m}
$$

is in the local Nilsson class on $\mathbb{D} \times \mathbb{D}^{\times}$too. Hence $a$ and $b$ have poles in $m$ of order at most 1 and their residues at $m=0$ cancel. Now use $\log (\epsilon)=s^{-1} \lim _{m \rightarrow 0}\left(m^{-1}\left(\epsilon^{s m}-1\right)\right)$.

This finishes the proofs of Theorem 7.2 and Theorem 7.9.

\section{References}

[AG] D.N. AkhiEZer, S.G. Gindikin, On Stein extensions of real symmetric spaces, Math. Ann. 286 (1990), 1-12.

[B] L. BARCHINI, Stein extensions of real symmetric spaces and the geometry of the flag manifold, Math. Ann. 326:2 (2003), 331-346.

[BeR] J. Bernstein, A. Reznikov, Analytic continuation of representations and estimates of automorphic forms, Ann. of Math. 150 (1999), 329-352.

[Bj] J.-E. BJöRK, Rings of Differential Operators, North-Holland Mathematical Library, North-Holland 1979.

[Bo et al] A. Borel, Et AL., Algebraic D-Modules, Perspectives in Mathematics, Academic Press, 1987.

[Bou] N. Bourbaki, Groupes et algébres de Lie, Chapitres 4,5 et 6, Hermann, Paris, 1968.

[Bu] D. Bump, Automorphic Forms on GL(3, R ), Springer LNM 1083, 1984.

[C] R. Carter, Finite Groups of Lie Type, Wiley Classics Library, John Wiley \& sons, 1993.

[Ch] I. Cherednik, A unification of Knizhnik-Zamolodchikov equations and Dunkl operators via affine Hecke algebras, Invent. Math. 106 (1991), 411432.

[D1] P. Deligne, Equations differentielles à points singulier regulier, Springer LNM 163 (1970).

[D2] P. Deligne, Les immeubles des groupes de tresses généralisés, Invent. Math. 17 (1972), 273-302.

[DuKV] J.J. Duistermaat, J.A.C. Kolk, V.S. Varadarajan, Functions, flows and oscillatory integrals on flag manifolds and conjugacy classes in real semisimple Lie groups, Comp. Math. 49 (1983), 309-398.

[Dun] C.F. DunkL, Differential-difference operators associated to reflection groups, Trans. Amer. Math. Soc. 311 (1989), 167-183.

[FH] G. Fels, A. Huckleberry, Characterization of cycle domains via Kobayashi hyperbolicity, Bull. Soc. Math. France 133:1 (2005), 121-144. 
[GL] S. Gelbart, E. LAPID, Lower bounds for $L$-functions at the edge of the critical strip, Amer. J. Math. 128 (2006), 619-638.

[GLS] S. Gelbart, E. LApid, P. SARnAK, A new method for lower bounds of $L$-functions, C.R. Acad. Sci. Paris Ser. I 339 (2004), 91-94.

[GS] S. Gelbart, F. Shahidi, Boundedness of automorphic $L$-functions in vertical strips, J. Amer. Math. Soc. 14:1 (2001),79-107.

[GiK1] S. Gindikin, B. KRötz, Complex crowns of Riemannian symmetric spaces and non-compactly causal symmetric spaces, Trans. Amer. Math. Soc 354:8 (2002), 3299-3327.

[GiK2] S. Gindikin, B. Krötz, Invariant Stein domains in Stein Symmetric spaces and a non-linear complex convexity theorem, IMRN 18 (2002), 959971.

[GoL] D. Goldfeld, X. LI, Voronoi Formulas on $G L(n$, preprint.

[H1] Harish-Chandra, Spherical functions on a semisimple Lie group I, Amer. J. Math. 80 (1958) 241-310.

[H2] Harish-Chandra, Automorphic Forms on Semisimple Lie Groups, Springer LNM 62 (1968).

[He] G.J. Heckman, An elementary approach to the hypergeometric shift operators of Opdam, Invent. Math. 103 (1991), 341-350.

[HeO] G.J. HeCKMan, E.M. Opdam, Root systems and hypergeometric functions I, Comp. Math. 64 (1987), 329-352.

[HeS] G.J. Heckman, H. Schlichtkrull, Harmonic Analysis and Special Functions on Symmetric Spaces, Academic Press, 1994.

[Hel] S. Helgason, Differential Geometry, Lie Groups, and Symmetric Spaces, Acad. Press, 1978

[HoM] R. Howe, C. Moore, Asymptotic properties of unitary representations, J. Funct. Anal. 32 (1979), 72-96

[Hu1] A. HuCKLEBERRY, On certain domains in cycle spaces of flag manifolds, Math. Ann. 323:4 (2002), 797-810.

[Hu2] A. HuCKLEBERRY, Erratum: On certain domains in cycle spaces of flag manifolds, to be submitted.

[Hum] J.E. HumphreYs, Introduction to Lie Algebras and Representation Theory, Second printing, revised. Graduate Texts in Mathematics 9. SpringerVerlag, New York-Berlin, 1978.

[K] V.G. KAC, Infinite Dimensional Lie Algebras, Progress in Mathematics 40, Birkhäuser, 1984.

[Ka1] S. KANEYUKI, On the subalgebras $\mathfrak{g}_{0}$ and $\mathfrak{g}_{\mathrm{ev}}$ of semisimple graded Lie algebras, J. Math. Soc. Japan 45:1, (1995), 1-19.

[Ka2] S. KANEYUKI, Signatures of Roots and a New Characterization of Causal Symmetric Spaces, Topics of Geometry: In memory of Joseph D'Atri (S. Gindikin, ed.), Progress in Nonlinear Differential Equations 20, Birkhäuser (1996), 213-229. 
[Kr] B. KRÖTz, Domains of holomorphy for irreducible unitary representations of simple Lie groups, MPIM preprint 102 (2006).

[KrS1] B. KRÖTz, R.J. Stanton, Holomorphic extensions of representations: (I) automorphic functions, Ann. of Math. 159 (2004), 641-742.

[KrS2] B. KRÖtz, R.J. Stanton, Holomorphic extensions of representations: (II) geometry and harmonic analysis, GAFA, Geom. funct. anal. 15 (2005), 190-245.

[KrO] B. Krötz, M. Oтто, A refinement of the complex convexity theorem via symplectic techniques, Proc. Amer. Math. Soc. 134:2 (2006), 549-558.

[KrOS] B. Krötz, G. Ólafsson, R. Stanton, The Image of the Heat Kernel Transform on Riemannian Symmetric Spaces of the Noncompact Type, IMRN 22 (2005), 1307-1329.

[L] R. LANGLANDS, On the functional equations satisfied by Eisenstein series, Springer LNM 544 (1976).

[Le] H. V.D. LEK, The homotopy type of complex hyperplane complements, Thesis, Univ. of Nijmegen (1983).

[M] I.G. Macdonald, Symmetric Functions and Hall Polynomials (second edition), Oxford Mathematical Monographs, 1995.

[Ma] B. Malgrange, Intégrales asymptotiques et monodomie, Annales scientifiques l'E.N.S. 4e série, tome 7:3 (1974), 405-430.

[Mat] T. Matsuki, Stein extensions of Riemann symmetric spaces and some generalization, J. Lie Theory 13:2 (2003), 565-572.

[Mi] H. LeE Michelson, A decomposition for certain real semisimple Lie groups, Trans. Amer. Math. Soc. 213 (1975), 177-193.

[MilS] S. Miller, W. Schmid, Automorphic distributions, L-functions, and Voronoi summation for GL(3), Ann. of Math. (2) 164:2 (2006), 423-488

[O1] E.M. OpdAM, Root systems and hypergeometric functions IV, Comp. Math. 67 (1988), 191-209.

[O2] E.M. OpDAM, An analogue of the Gauss summation formula for hypergeometric functions related to root systems, Math. Z. 212 (1993), 313-336.

[O3] E.M. OPDAM, Dunkl operators, Bessel functions and the discriminant of a finite Coxeter group, Compositio Math. 85 (1993), 333-373.

[O4] E.M. OPDAM, Harmonic analysis for certain representations of graded Hecke algebras, Acta. Math. 175 (1995), 75-121.

[O5] E.M. Opdam, Lecture Notes on Dunkl Operators for Real and Complex Reflection Groups, MSJ Memoirs 8, Mathematical Society of Japan (2000).

[P] Y. PETRIDIs, On squares of eigenfunctions for the hyperbolic plane and a new bound on certain $L$-series, IMRN 3 (1995), 111-127.

[S] P. SARNAK, Integrals of products of eigenfunctions, IMRN 6 (1994), 251260.

[Sh] J. ShalikA, The multiplicity one theorem for $\mathrm{GL}_{n}$, Ann. of Math. (2) 100 (1974), 171-193. 
[St1] R. Steinberg, Differential equations invariant under finite reflection groups, Trans. Amer. Math. Soc. 112 (1964), 392-400.

[St2] R. SteinberG, Endomorphisms of Linear Algebraic Groups, Memoirs of the Amer. Math. Soc. 80, American Mathematical Society, Providence R.I., 1968.

[W] W. WALTER, Gewöhnliche Differentialgleichungen, Heidelberger Taschenbücher 110, Springer-Verlag, Berlin-Heidelberg-New York (1972).

Bernhard KröTz, Max-Planck-Institut für Mathematik, Vivatsgasse 7, D-53111 Bonn, Germany

kroetz@mpim-bonn.mpg.de

Eric Opdam, Korteweg de Vries Institute for Mathematics, University of Amsterdam, Plantage Muidergracht 24, 1018TV Amsterdam, The Netherlands

opdam@science.uva.nl

Received: August 2006

Revision: June 2007

Accepted: June 2007 